\title{
Molecular mechanisms of acute axonal degeneration in the rat optic nerve
}

\author{
Dissertation \\ for the award of the degree \\ "Doctor of Philosophy" (Ph.D.) \\ Division of Mathematics and Natural Sciences \\ of the Georg-August-Universität Göttingen \\ submitted by \\ Jiannan Zhang \\ born in Tianjin, China
}

Göttingen 2015 


\section{Thesis committee:}

Prof. Dr. Ralf Heinrich (Department of Cellular Neurobiology, Schwann-Schleiden Research Centre, University of Göttingen)

Prof. Dr. Michael Hörner (Johann-Friedrich-Blumenbach-Institute for Zoology and Anthropology, Department of Cellular Neurobiology, University of Göttingen)

Prof. Dr. Paul Lingor (Department of Neurology, University Medical Center Göttingen)

\section{Members of the Examination Board:}

First Reviewer: Prof. Dr. Ralf Heinrich (Department of Cellular Neurobiology, Schwann-Schleiden Research Centre, University of Göttingen)

Second Reviewer: Prof. Dr. Michael Hörner (Johann-Friedrich-Blumenbach-Institute for Zoology and Anthropology, Department of Cellular Neurobiology, University of Göttingen)

\section{Further members of the Examination Board:}

Prof. Dr. Tiago Fleming Outeiro (Department of NeuroDegeneration and Restaurative Research, University Medical Center Göttingen)

Prof. Dr. Martin Göpfert (Department of Cellular Neurobiology, Schwann-Schleiden Research Centre, University of Göttingen)

Dr. Sebastian Kügler (Department of Neurology, University Medical Center Göttingen)

Dr. Camin Dean (European Neuroscience Institute, Göttingen)

Date of the oral examination: 11th of November, 2015 


\section{Declaration}

I hereby declare that the thesis:

"Molecular mechanisms of acute axonal degeneration in the rat optic nerve"

has been written independently and with no other sources and aids than quoted.

Jiannan Zhang

Göttingen, October 2015 


\section{Contents}

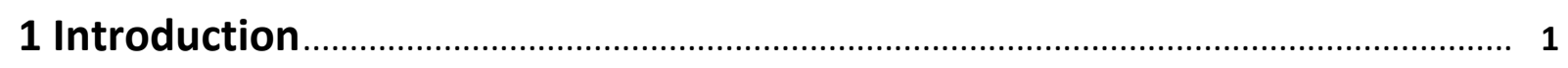

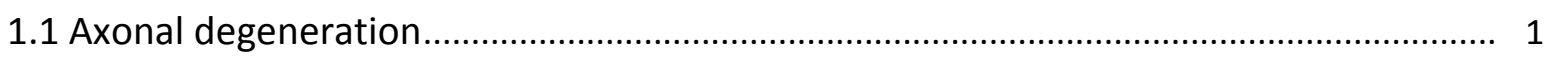

1.1.1 Developmental axonal degeneration ............................................................. 1

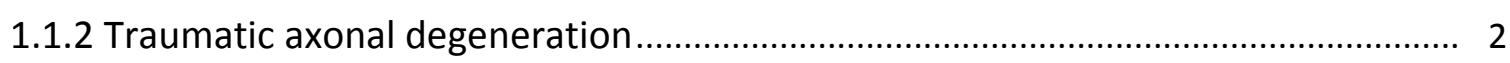

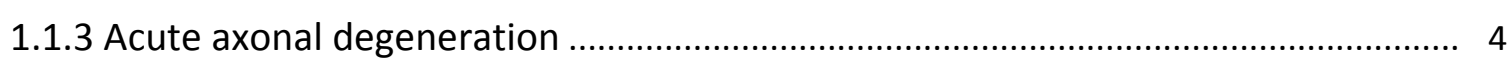

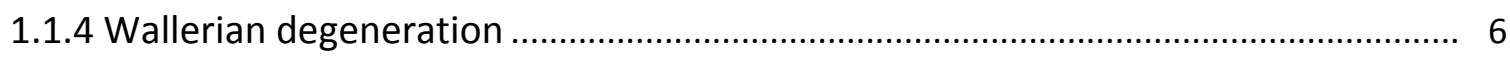

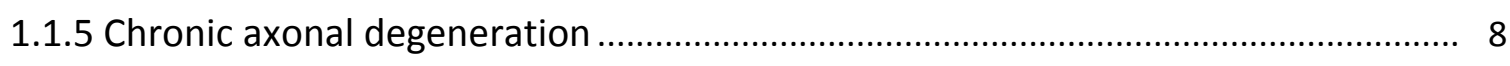

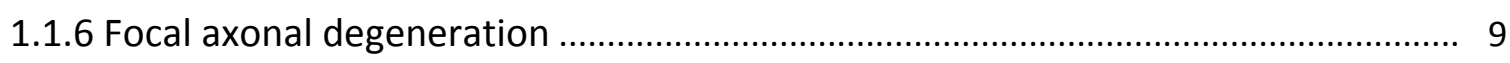

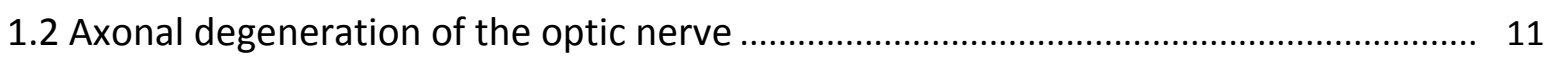

1.2.1 Axonal degeneration in diseases affecting the optic nerve ................................. 11

1.2.2 The optic nerve as a model system for axonal degeneration in the CNS .............. 12

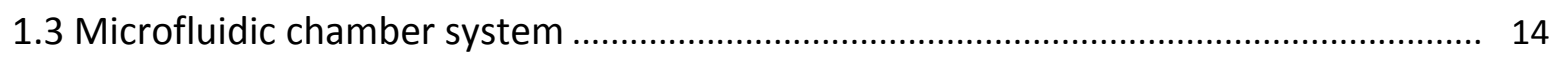

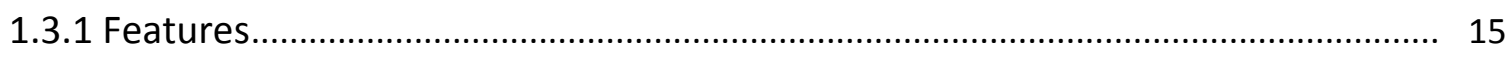

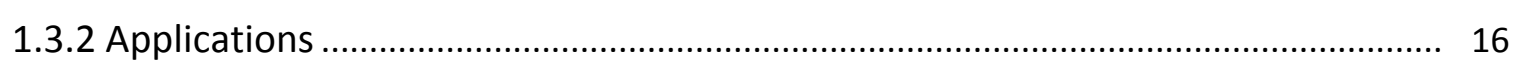

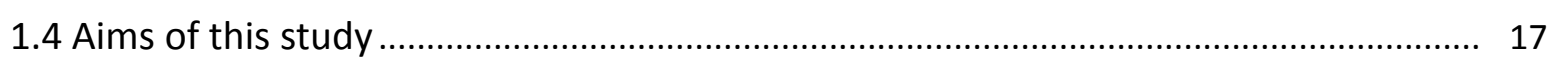

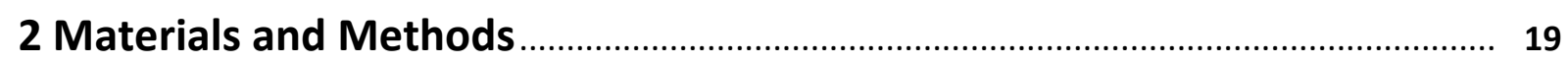

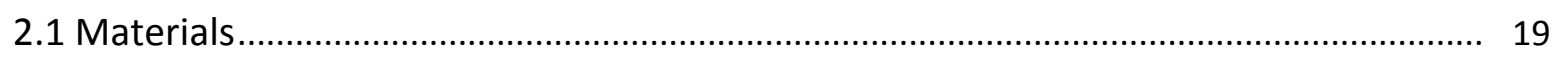

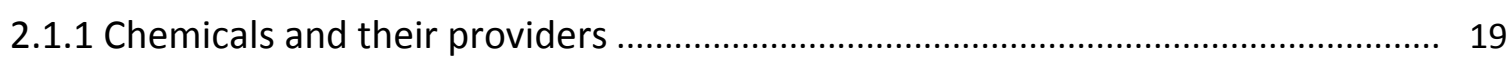

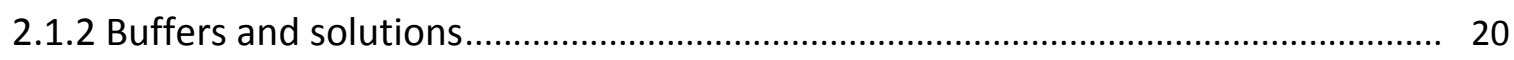

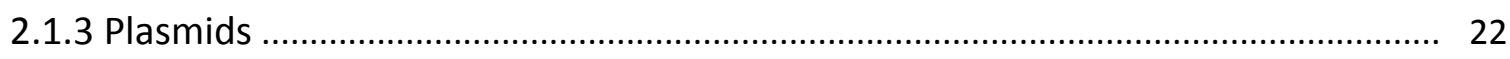

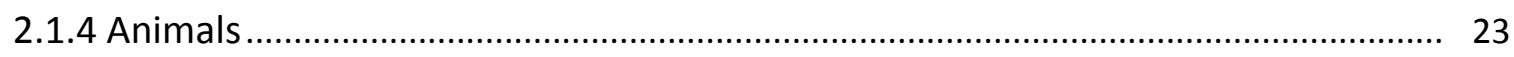

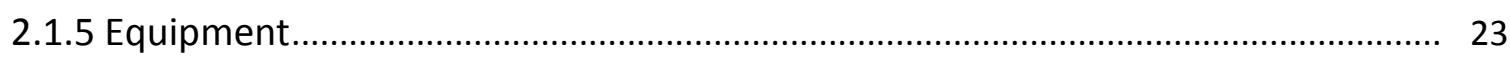

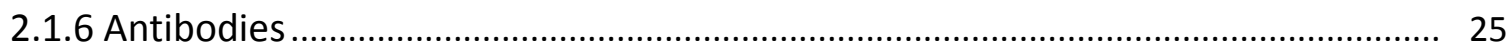

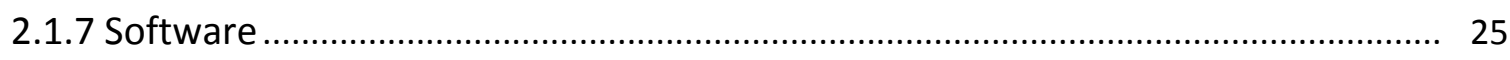

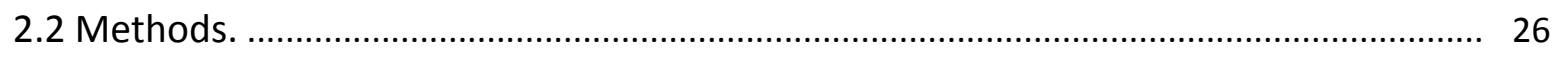

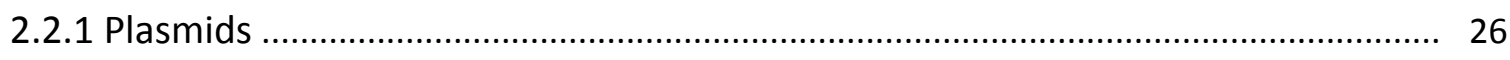

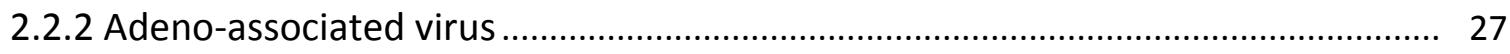


2.2.3 Surgical procedures

2.2.4 Primary cortical neuron culture ......................................................................... 30

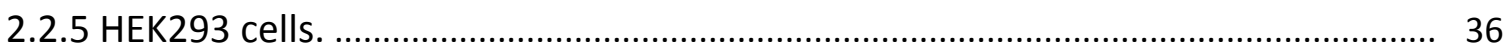

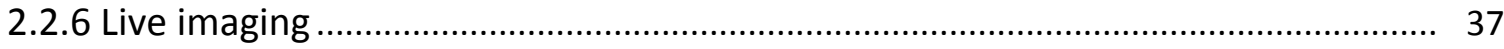

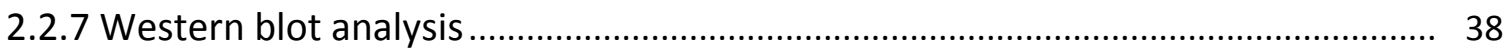

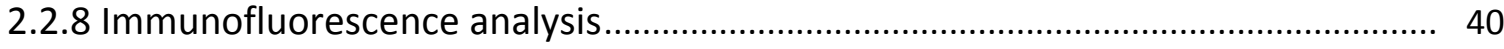

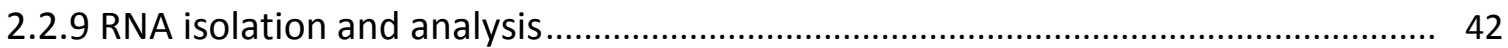

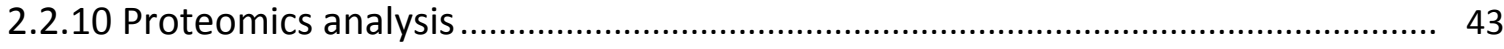

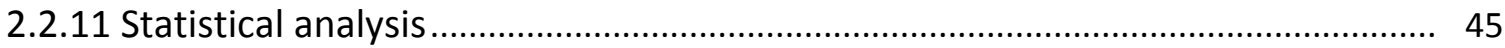

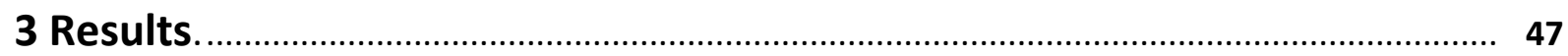

3.1 The role of calpain in acute axonal degeneration of the optic nerve ........................... 47

3.1.1 Time course and localization of calpain activation during acute axonal

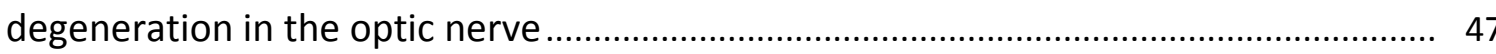

3.1.2 Live imaging of crush-induced acute axonal degeneration after calpain inhibition in vivo

3.1.3 Characterization of downstream targets of calpain during acute axonal

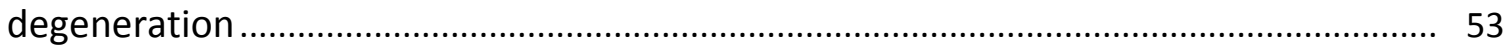

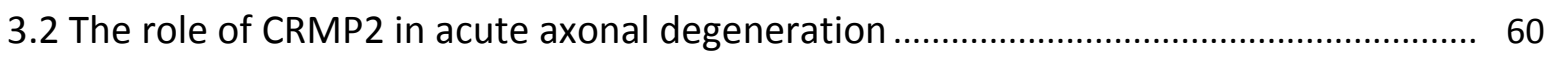

3.2.1 Establishment of an in vitro acute axonal degeneration model in the microfluidic

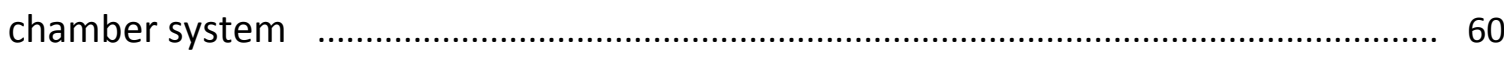

3.2.2 Effects of CRMP2 overexpression on axonal degeneration after axotomy of cortical

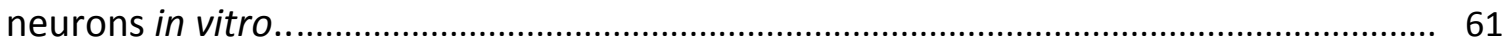

3.2.3 Overexpression of CRMP2 mediated by the viral vector AAV.CRMP2 ................... 65

3.2.4 Live imaging of crush-induced acute axonal degeneration in the rat optic nerve in vivo after AAV-mediated CRMP2 overexpression........................................................ 68

3.2.5 Effects of CRMP2 overexpression on mitochondrial transport after axotomy...... 70

3.3 Proteomics analysis of acute axonal degeneration in the optic nerve ......................... 73

4 Discussion. 78

4.1 The role of calpain in acute axonal degeneration of the optic nerve in vivo................ 78

4.1.1 Calpain is activated early during acute axonal degeneration ................................. 79 
4.1.2 Calpain inhibition attenuates crush-induced acute axonal degeneration in vivo. 80

4.1.3 CRMP2 is an important downstream target of calpain during acute axonal degeneration

4.2 The role of CRMP2 in acute axonal degeneration in vitro and in vivo

4.2.1 CRMP2 overexpression delays acute axonal degeneration

4.2.2 CRMP2 overexpression rescues the impairment of mitochondrial transport during acute axonal degeneration.

4.3 Proteomics analysis of acute axonal degeneration in the optic nerve. 85

4.4 Conclusion 86

5 Summary. 88

6 References. 89

Abbreviations. 103

Acknowledgements. 106

Curriculum vitae. 108 


\section{Introduction}

\subsection{Axonal degeneration}

Axonal degeneration is a prominent pathological feature in many neurological diseases including neurodegenerative diseases, chronic inflammatory diseases and traumatic injuries of nerves, spinal cord or brain (Coleman, 2005). It often occurs already in the early disease course and precedes the death of the cell body (Coleman, 2005). Axonal degeneration often results in irreversible defects in neuronal connectivity, leading to persistent functional deficits with high clinical relevance (Jawhar et al., 2012; Oakley et al., 2006). It is an active biological process independent of cell death and controlled by distinct molecular mechanisms (Adalbert et al., 2006; Johnson, 1994). As axonal damage correlates with permanent clinical deficits and axons have the potential to regenerate, therapeutic approaches at this time point should focus more on the axonal compartment instead of following purely anti-apoptotic strategies aimed at the neuronal soma. Therefore, a better understanding of the mechanisms of axonal degeneration is mandatory to develop effective treatments for neurological diseases.

Different forms of axonal degeneration have been described: physiological axonal degeneration during development, different stages of axonal degeneration following a traumatic nerve injury and chronic axonal degeneration in neurodegenerative and neuroinflammatory diseases.

\subsubsection{Developmental axonal degeneration}

Axonal degeneration occurs physiologically in the development of the nervous system (Luo and O'Leary, 2005). During early development, an exuberant number of axons are formed. Based on successful synapse formation, functionality and yet unknown molecular signals, persisting axonal connections are selected, while all unnecessary connections are pruned by axonal degeneration in later development (Luo and O'Leary, 2005). This process is best studied in the retinotectal system. In the developing chick, for example, all retinotectal axons, 
that do not grow directly to their appropriate terminal zone are eliminated later through axonal degeneration (Nakamura and O'Leary, 1989). In the developing neocortex of mice, pruning of exuberant long branches of thalamocortical axons also occurs by actively driven axonal degeneration (Luo and O'Leary, 2005).

Developmental axonal degeneration often occurs within a short period leading to large-scale elimination of axonal segments (Luo and O'Leary, 2005; Nakamura and O'Leary, 1989). Morphologically it is similar to the rapid fragmentation of distal axons during axonal degeneration after axonal injury in the adult animal (Waller, 1850). The morphological similarities suggest that they may share some common mechanisms. Indeed, both the ubiquitin-proteasome system and the glial cell surface receptor Draper are required for axonal degeneration during development and after injury (Hoopfer et al., 2006). However, at the same developmental age of flies or mice, overexpression of the Wlds protein inhibits injury-induced axonal degeneration while it does not affect developmental axonal degeneration of the same axons (Hoopfer et al., 2006). Thus, these two types of axonal degeneration differ in the early stage while they have similar late stage execution of axon degeneration (Hoopfer et al., 2006). Further studies are needed to investigate which molecular signals govern developmental axonal degeneration and how diverse factor-triggered axonal degeneration leads to a common execution pathway.

\subsubsection{Traumatic axonal degeneration}

A lesion of axons, e.g. in the spinal cord or optic nerve, leads to traumatic axonal degeneration (Figure 1.1), which can result in a permanent dysfunction of the affected nerve tract. During traumatic axonal degeneration, the axons undergo two morphologically different phases of degeneration, separated by a lag phase. Within several hours after lesion, axons in both proximal and distal parts undergo a rapid fragmentation, which is termed 'acute axonal degeneration' (AAD) (Kerschensteiner et al., 2005; Knöferle et al., 2010) (see 1.1.3 Acute axonal degeneration). After AAD, the distal axon stays morphologically stable for a short period. After 24 to 72 hours after lesion, Wallerian degeneration (WD) occurs, in which the distal part of the lesioned axon is fragmented (Conforti et al., 2014; Waller, 1850) 


\section{(see 1.1.4 Wallerian degeneration)}

Traumatic axonal degeneration has been studied extensively. This is not only due to its clinical relevance but also to its reproducibility and convenient experimental accessibility (Raff et al., 2002; Wang et al., 2012). Moreover, traumatic axonal degeneration and axonal pathology in chronic neurological diseases share some common morphological features including axonal swellings, cytoskeleton disassembly, and axonal fragmentation (Cavanagh, 1964; Kerschensteiner et al., 2005). They also share convergent molecular mechanisms. For example, mitochondrial transport is impaired in both axotomy-induced WD and chronic axonal degeneration in ALS models (Avery et al., 2012; De vos et al., 2007). Moreover, a recent study showed that overexpression of alpha-synuclein, a protein involved in chronic PD pathogenesis, accelerates the time course of AAD in the optic nerve (Koch et al., 2015). Thus, studying the mechanisms of traumatic axonal degeneration could help to understand also the axonal pathology in chronic neurological diseases (Coleman, 2005; Wang et al., 2012). 


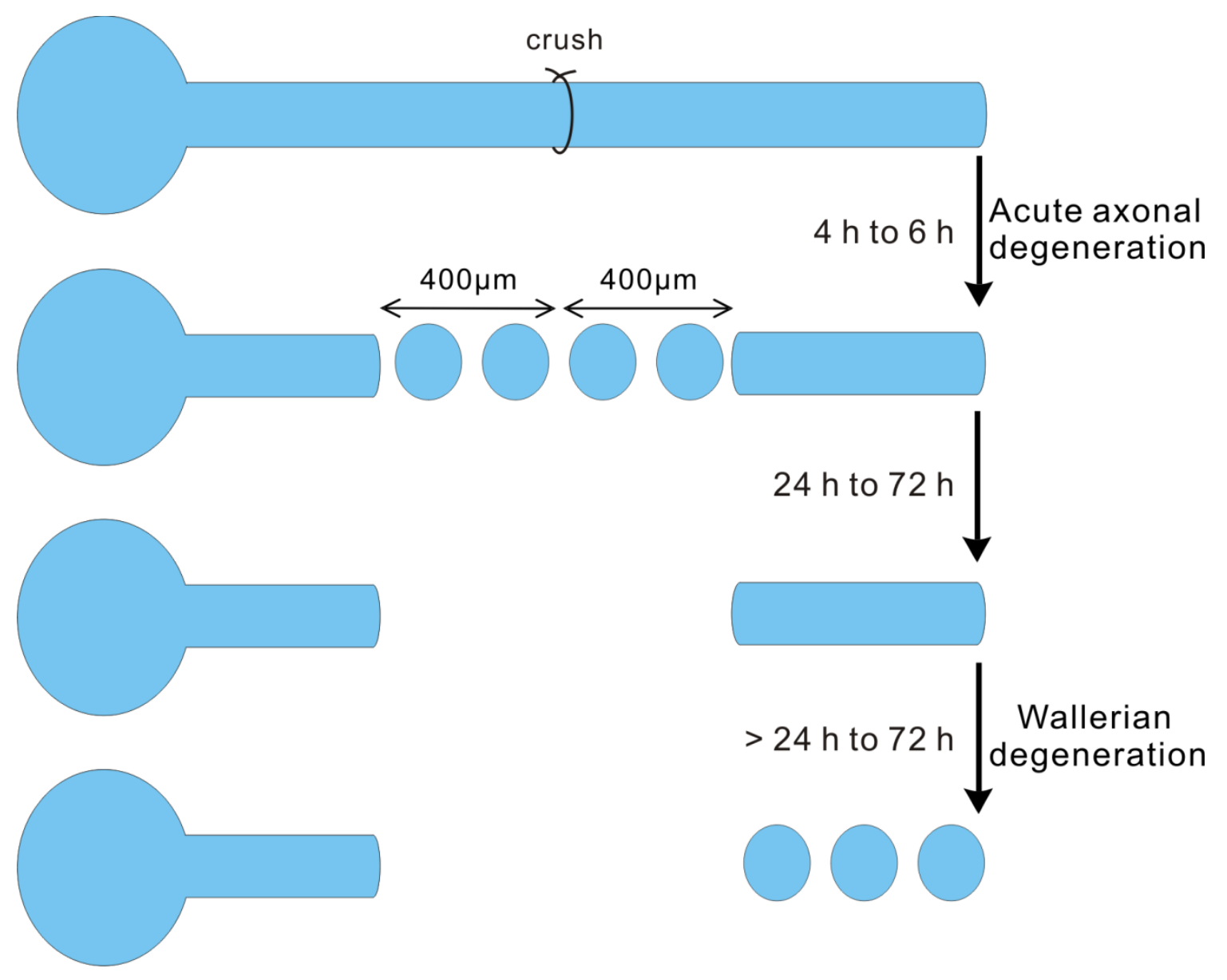

Figure 1.1 Traumatic axonal degeneration in the central nervous system.

After a traumatic injury of an axon in the central nervous system, acute axonal degeneration occurs within the first $6 \mathrm{~h}$ after injury. Axons undergo rapid fragmentation in the region $400 \mu \mathrm{m}$ proximal and distal to the lesion site. Afterwards, the axons stay morphologically stable for a short period. At $24 \mathrm{~h}$ to $72 \mathrm{~h}$ after lesion, Wallerian degeneration occurs, in which the distal part of the axon undergoes fragmentation.

\subsubsection{Acute axonal degeneration}

AAD occurs within several hours after a traumatic lesion of CNS axons and was first described after spinal cord injury (Kerschensteiner et al., 2005) and further studied after optic nerve crush (ONC) (Knöferle et al., 2010). In both spinal cord and optic nerve models, the process of $A A D$ was visualized by in vivo live imaging techniques. Morphologically, $A A D$ is characterized by a rapid axonal disintegration in a time dependent manner on both proximal 
and distal sides of the lesion comprising the adjacent $400 \mu \mathrm{m}$. Bulb-like swellings are observed in the axons within the first hour after lesion, followed by axonal fragmentation. Both proximal and distal sides of the lesion have a similar kinetics of degeneration.

At the ultrastructural level, condensation and misalignment of neurofilaments were observed within $30 \mathrm{~min}$ after ONC and followed by the fragmentation of microtubules (Knöferle et al., 2010). Axonal swellings were already detected at $30 \mathrm{~min}$ after ONC. These swellings were characterized by accumulated organelles, mostly mitochondria and vacuoles, although different phenotypes of axonal bulbs have been described. The local accumulation of organelles suggests an impairment of axonal transport, which is, however, not experimentally proven so far. Accumulated mitochondria displayed signs of degradation such as swellings, suggesting a local mitochondrial dysfunction. A large portion of vacuoles morphologically resembled autophagosomes. Indeed, this type of vacuoles was labeled by the autophagy induction marker microtubule-associated protein 1 light chain 3 (LC3) using immunogold staining (Knöferle et al., 2010).

On the molecular level, an intra-axonal calcium increase is the initial crucial event during AAD (Knöferle et al., 2010). By using a calcium-sensitive dye, a calcium increase in the axons was observed within $40 \mathrm{~s}$ after ONC. The calcium levels returned to basic levels in the next minute. Local application of a mixture of calcium channel inhibitors completely inhibited the increase of axonal calcium. This suggests that extracellular calcium enters the axons through calcium channels and thereby leads to increased levels of intra-axonal calcium. Furthermore, application of calcium inhibitors prevented the process of AAD while calcium ionophore aggravated degeneration, demonstrating the important role of calcium influx.

During AAD of the spinal cord it was shown that the calcium dependent protease calpain is activated $400 \mu \mathrm{m}$ proximal and distal to the lesion site at $30 \mathrm{~min}$ after spinal cord transection (Kerschensteiner et al., 2005). Treatment with calpain inhibitors completely blocked axonal fragmentation within $1 \mathrm{~h}$ after spinal cord injury. However, the detailed kinetics of calpain activation, its relevant molecular targets and the long-term effects of calpain inhibition during $A A D$ were not studied so far.

Another feature of $A A D$ on the molecular level is the induction of autophagy following the 
initial calcium increase (Knöferle et al., 2010). The number of autophagosomes started to increase at $30 \mathrm{~min}$ after ONC and was doubled at $6 \mathrm{~h}$ after ONC compared to the native optic nerve. Pharmacological inhibition of autophagy reduced the number of autophagosomes induced by ONC and attenuated axonal disintegration. However, the protective effect of autophagy inhibition during AAD was not as pronounced as the one achieved by calcium inhibition. Interestingly, application of the calcium inhibitor mix reduced the number of autophagosomes during $A A D$, suggesting that the production of autophagosomes is triggered by the initial increase of axonal calcium and that autophagy induction is a downstream target of calcium influx.

\subsubsection{Wallerian degeneration}

At 24 to 72 hours after a traumatic axonal lesion, the distal part of the axon that is not affected by $A A D$ undergoes a rapid fragmentation, which finally leads to the complete removal of the distal axon (Waller, 1850). This degenerative process is termed WD and was first described after lesion of glossopharyngeal and hypoglossal nerves in the frog (Waller, 1850). WD proceeds in a speed range from $0.4 \mathrm{~mm} / \mathrm{h}$ to $24 \mathrm{~mm} / \mathrm{h}$ (Lingor et al., 2012), which depends on axonal diameter, length of distal stumps and species (Beirowski et al., 2005; Rotshenker, 2011).

For decades, the slow Wallerian degeneration (Wlds) mutant mouse has been used as a tool to investigate the mechanisms of WD. In the Wlds mutant mouse, the process of WD is robustly delayed. For example, the axonal cytoskeleton in the sectioned peripheral nerve in Wlds mutant mice was still intact 5 days after injury while that from wild-type mice disintegrated 3 days after injury (Lunn et al., 1989). This mutant expresses the Wlds protein, which is responsible for slowing down the process of WD. Wlds protein consists of full-length nicotinamide mononucleotide adenylyltransferase-1 (NMNAT1) and the N-terminal fragment of ubiquitination factor E4B (UBE4B) (Mack et al., 2001). Overexpression of NMNAT1 alone is sufficient to prevent axonal degeneration in dorsal root ganglion (DRG) cells in vitro and in mice in vivo (Araki et al., 2004; Sasaki et al., 2009). This suggests that NMNAT1 is the most important functional component of the Wlds protein. 
Recently, a study using small interfering RNA (siRNA) has shown that knockdown of endogenous NMNAT1 does not induce WD in uninjured axons. However, knockdown of its homologue NMNAT2 is sufficient to induce WD of uninjured axons (Gilley and Coleman, 2010). Furthermore, endogenous NMNAT2 is rapidly degraded in injured distal axons of cultured neurons shortly before WD is initiated (Conforti et al., 2014; Gilley and Coleman, 2010). Besides, overexpression of exogenous NMNAT2 protects transfected axons against WD (Gilley and Coleman, 2010). All these results demonstrate that NMNAT2 is the most important molecule, preventing WD when constantly supplied and inducing WD when depleted in the healthy wildtype mammalian axon (Gilley and Coleman, 2010). Moreover, the pro-degenerative molecules SARM1 and PHR1 were described to play important roles in the molecular cascade of WD (Conforti et al., 2014).

Downstream of both NMNAT1 and NMNAT2 in WD is a local increase of intraaxonal calcium (Adalbert et al., 2012; Yang et al., 2013). Increased intraaxonal calcium results in the subsequent activation of the calcium dependent protease calpain (Ma et al., 2013). Besides, mitochondrial changes have been described in the pathology of WD (Avery et al., 2012).

$A A D$ is clearly different from WD in terms of when and where it occurs after a traumatic lesion. AAD occurs within several hours after a traumatic lesion while WD proceeds at 24-72 $\mathrm{h}$ after lesion. AAD affects both proximal and distal parts equally while WD only affects the distal part. The final goal of therapeutic approaches to axonal degeneration is to promote regeneration of the proximal axon stump. Thus interference with AAD has a considerable clinical relevance as it could stabilize the remaining proximal axon. WD, on the other hand, is probably a necessary prerequisite for later axonal regeneration, as the axonal tracts within the myelin sheaths need to be cleared to let the new axon grow through.

However, AAD and WD also share some common features. The speed of fragmentation, size of fragments, and the spacing between fragments is similar in WD and AAD (Kerschensteiner et al., 2005). The expression of the Wlds protein, which delays WD, also largely protected axons from AAD (Kerschensteiner et al., 2005). Furthermore, both the increase of intraaxonal calcium and calcium dependent events play an important role in WD and AAD (George et al., 1995; Kerschensteiner et al., 2005; Knöferle et al., 2010). 


\subsubsection{Chronic axonal degeneration}

'Dying back' axonal degeneration

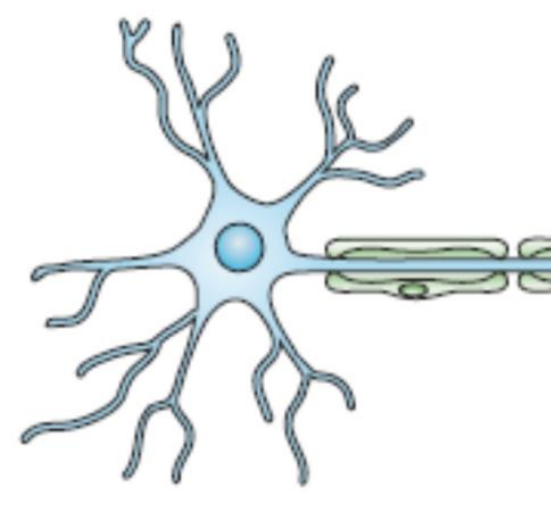

Distally degenerated nerves
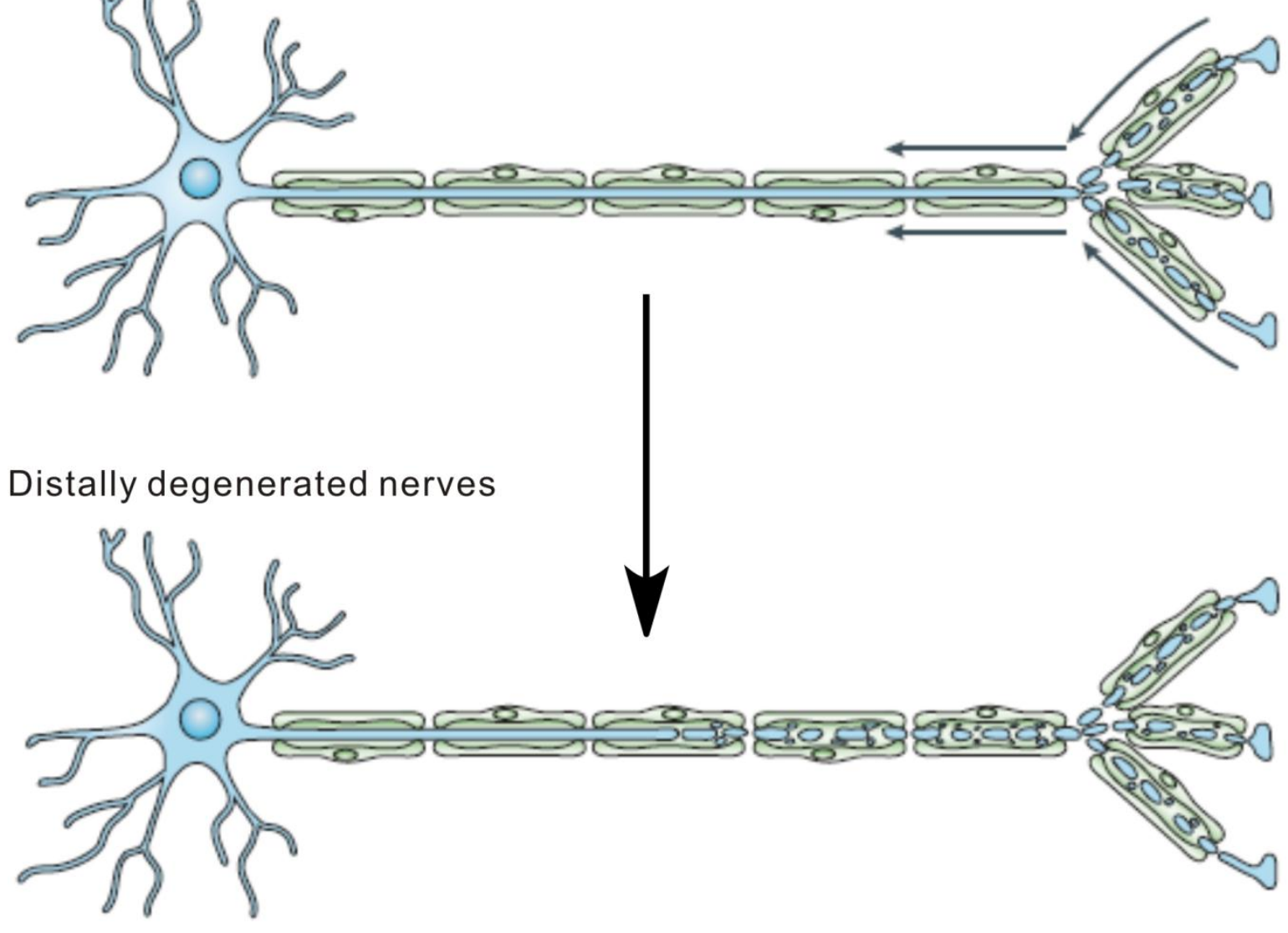

Figure 1.2 Dying back axonal degeneration in neurodegenerative diseases.

Dying back degeneration starts at the synaptic terminals or the distal axons and then progresses gradually towards the cell body (modified from Coleman, 2005).

Chronic axonal degeneration refers to the degenerative process of axons in chronic neurological diseases. All neurodegenerative diseases, such as PD (Orimo et al., 2008), Alzheimer's disease (AD) (Selkoe, 2002) and amyotrophic lateral sclerosis (ALS) (Nihei et al., 1993), show axonal pathology in the form of 'dying back' (Cavanagh, 1964). Dying back degeneration starts at the synaptic terminals or the distal axons, and then progresses gradually towards the cell body (Figure 1.2). Consistent with this, axonal terminals of the nigrostriatal pathway are affected much earlier than cell bodies in PD (Burke, 2014). Lewy 
bodies as the histological hallmark of PD are initially found in the distal axons and only later in the cell bodies and proximal neurites (Orimo et al., 2008). The morphological features of dying back degeneration include axonal swellings, microtubule disassembly, and the final axonal fragmentation (Arduíno et al., 2013; Öztürk et al., 2013; Tagliaferro et al., 2015). Regarding the underlying mechanisms, mitochondrial dysfunction (Shi et al., 2010), synaptic pathology (Morales et al., 2015) as well as disruption of axonal transport (Bilsland et al., 2010; Morfini et al., 2007) have been described in dying back degeneration. For instance, anterograde transport of mitochondria was reduced in the motor neurons isolated from ALS mice (De vos et al., 2007). Axonal transport impairment might explain the formation of axonal swelling during dying back axonal degeneration. Besides, a recent study showed that dying back degeneration in dopaminergic neurons was mediated by increased macroautophagic activity (Cheng et al., 2011).

\subsubsection{Focal axonal degeneration}

Recently, another form of axonal degeneration termed 'focal axonal degeneration' (FAD) was visualized in multiple sclerosis (MS) (Figure 1.3) (Craner and Fugger, 2011; Nikić et al., 2011). In the experimental autoimmune encephalomyelitis (EAE) mouse model of MS, axonal degeneration was observed as an important feature and even began within the silent period of the disease (Wang et al., 2005). During FAD, a focal swelling containing accumulated organelles and mitochondria was observed at the beginning, which finally led to multifocal and bidirectional fragmentation of the axon (Nikić et al., 2011). Notably, the focal intraaxonal mitochondrial pathology was the earliest ultrastructural change and preceded the axonal and later glial pathology although demyelination is the hallmark of MS (Nikić et al., 2011). The mitochondrial pathology was induced by the increase of reactive oxygen and nitrogen species (ROS and RNS), which were derived from macrophages or activated microglia after acute EAE lesion (Nikić et al., 2011). Interestingly, during FAD, a proportion of axons spontaneously recovered in the early stages of FAD, which might explain some spontaneous remissions of symptoms in MS (Nikić et al., 2011). The thinner axons showed a higher vulnerability to FAD (Craner and Fugger, 2011). Similar ultrastructural axonal changes 
consistent to FAD were observed in human tissue from patients with MS (Nikić et al., 2011).

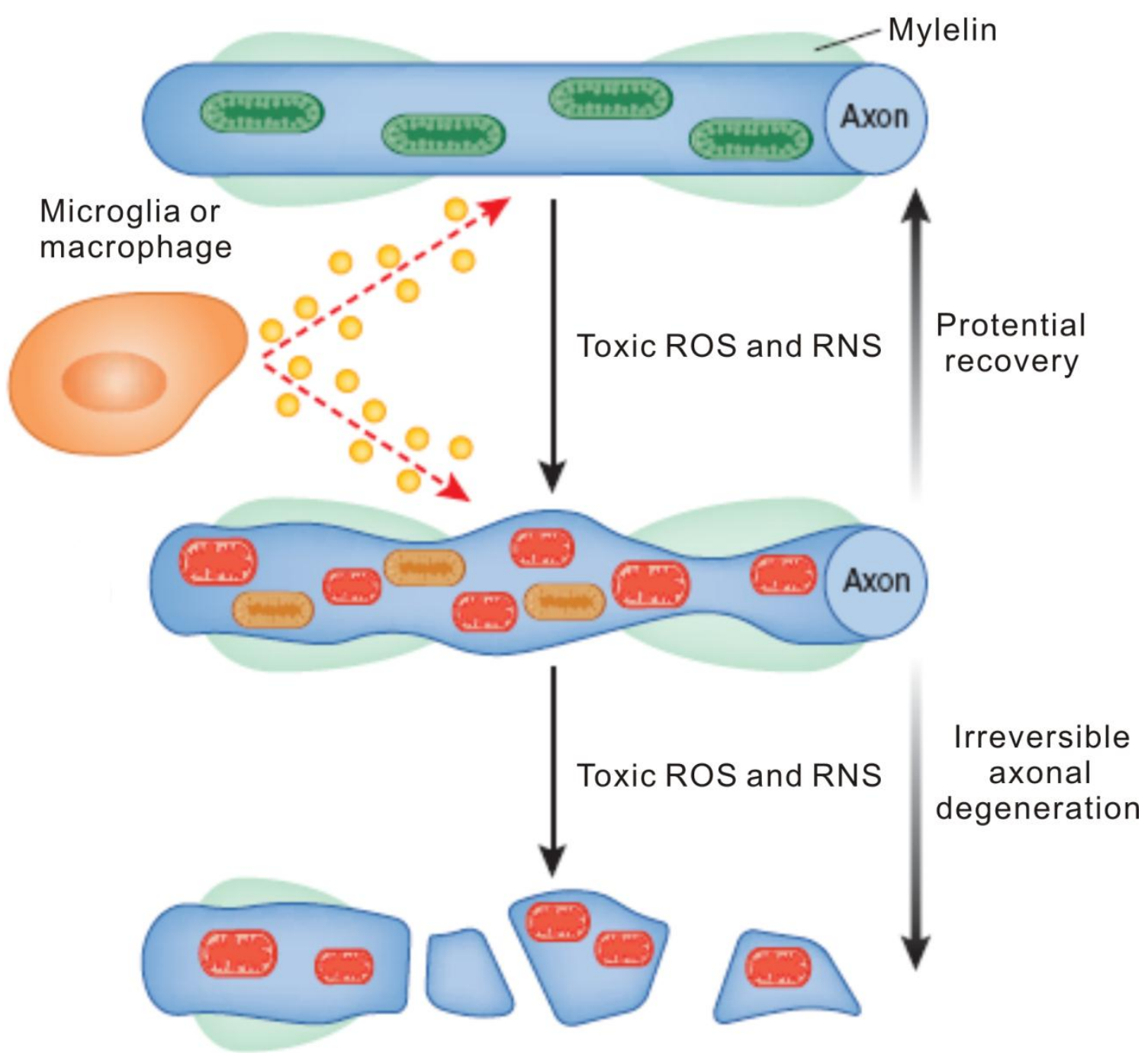

Figure 1.3 Focal axonal degeneration in neuroinflammatory diseases.

In the acute experimental autoimmune encephalomyelitis (EAE) mouse model, activated macrophages or microglia release reactive oxygen and nitrogen species (ROS and RNS), which induce mitochondrial pathology. At the early stage of focal axonal degeneration (FAD), the axons show focal swellings containing accumulated abnormal mitochondria. A proportion of such axons can spontaneously recover. However, due to continuous exposure to ROS and RNS, other axons proceed to irreversible degeneration characterized by multifocal and bidirectional fragmentation (modified from Craner and Fugger, 2011). 


\subsection{Axonal degeneration of the optic nerve}

Axonal degeneration of the optic nerve is a critical event in several pathological conditions including glaucoma, optic neuritis, hereditary optic nerve atrophy and traumatic injury (Ghaffarieh and Levin, 2012; You et al., 2013). The degeneration of retinal ganglion cell (RGC) axons is often followed by the death of the neuronal soma, leading to visual dysfunction or even complete vision loss. Besides, the optic nerve has been used extensively as a model system to study the degeneration of CNS axons.

\subsubsection{Axonal degeneration in diseases affecting the optic nerve}

Axonal degeneration plays an important role in glaucoma, traumatic eye diseases and inherited optic nerve diseases like dominant optic atrophy (DOA).

Glaucoma is the leading cause of blindness worldwide (Resnikoff et al., 2008). It is associated with an elevated intraocular pressure (IOP). Besides, there is increasing evidence that the development of glaucoma involves the inherent degeneration of RGC axons (Ghaffarieh and Levin, 2012). In agreement with this, both mean axonal density and total axon number were decreased in optic nerve sections from human patients and in an experimental glaucoma model (Mabuchi et al., 2004). In a chronic glaucoma mouse model (DBA/2J), an accumulation of organelles in RGC axons was observed as the first sign of axonal damage in glaucoma (Howell et al., 2007). Further studies showed that the axonal degeneration in glaucoma occurs in the forms of dying back and Wallerian axonal degeneration (Howell et al., 2007), sharing similar molecular mechanisms with neurodegenerative diseases and WD (McKinnon, 2012). For example, an impairment of axonal transport has been described as an early event during glaucoma, WD and neurodegenerative diseases (Diekmann and Fischer, 2013).

The genetic disease DOA affects one in every 12,000 people (Alavi et al., 2007). The patients often suffer from moderate vision loss, which is mainly caused by axonal degeneration of the optic nerve (Alavi et al., 2007; Lenaers et al., 2012). Axonal loss was already detected at early disease stages (Milea et al., 2010). In an animal model of DOA, 
axonal swellings as well as a reduced number of axons were also observed (Alavi et al., 2007).

Traumatic optic diseases involve a degeneration of RGC axons in the form of traumatic axonal degeneration (see 1.1.2 Traumatic axonal degeneration). The degeneration of RGC axons leads to the later death of RGC. In adult rats, RGC started to die at 5 days after intraorbital optic nerve transection, and about 50\% cells died at 1 week after the lesion. By 2 weeks after the lesion, only less than $10 \%$ RGC cells had survived. Both axonal degeneration and the death of RGCs lead to visual impairment or loss (Bähr, 2000). Thus, understanding the mechanisms on traumatic axonal degeneration of the optic nerve is necessary for developing effective therapeutic strategies.

Besides, axonal degeneration is associated with optic inflammatory diseases such as optic neuritis (Petzold et al., 2004; Trip, 2005).

\subsubsection{The optic nerve as a model system for axonal degeneration in the CNS}

The optic nerve has been used extensively as a model system to study axonal degeneration in the CNS. The optic nerve belongs to the CNS and axonal pathologies in optic neuropathies share similar mechanisms with other neurodegenerative diseases of the CNS. Importantly, the optic nerve has a convenient surgical accessibility and well-defined anatomy. Moreover, it offers the possibility to manipulate RGC axons by intravitreal injection of viral vectors or pharmacological substances.

Different injury models have been used to study the molecular mechanisms of axonal degeneration in the optic nerve. The complete injury or axotomy model involves the transection of the optic nerve. The exposed optic nerve is usually transected about $2 \mathrm{~mm}$ from the posterior eye pole with a knife or scissor (Lingor et al., 2005, 2008). However, after a complete transection, the lesioned optic nerve is separated into two parts and the anatomical structure of the nerve, including myelin and meninges, is completely destroyed. This model is thus suited to study degeneration and RGC death but not appropriate for axonal regeneration studies as regenerating axons usually need some lead structures. Recently, a partial optic nerve transection model was established using a new instrument 
called optic nerve quantitative amputator (Wang et al., 2012). Using this instrument, the semi-transection of the optic nerve is controlled quantitatively. This is achieved by a fixation of the exposed optic nerve in a stable position and removal of the upper half of the optic nerve along a cutting groove. This partial transection model maintains the meninges, and is suitable for studying oriented growth and regeneration of optic nerve axons. There are also incomplete injury models of the optic nerve such as crush and stretch. Stretch injury of the optic nerve is performed by rapid elongation of the nerve and is a suitable model to study diffuse axonal injury in the CNS (Gennarelli et al., 1989; Saatman et al., 2003). The crush lesion of the optic nerve is achieved by different techniques such as clips, forceps or sutures (Cai et al., 2012; Cho et al., 2005). In our group, a surgical suture is used to perform ONC, which leads to the complete transection of axons while leaving the anatomical structure of the optic nerve intact (Knöferle et al., 2010; Koch et al., 2013; Lingor et al., 2007). This model is suitable for studying axonal degeneration and regeneration after axotomy (Knöferle et al., 2010).

Besides these injury models, several optic disease models have been used to study axonal degeneration in the optic nerve. For example, DBA/2J mice and IOP-based animal models have been used to study glaucoma (Levkovitch-Verbin, 2004). The EAE animal model has been used to study axonal degeneration in optic neuritis and MS (Levkovitch-Verbin, 2004). In another mouse model, mitochondrial complex I was inhibited by intravitreal injection of the natural pesticide rotenone (Zhang et al., 2002). This model was used to study hereditary optic neuropathy and the neurodegenerative diseases linked to mitochondrial dysfunction (Levkovitch-Verbin, 2004).

The optic nerve is also well-suited to study axonal degeneration by in vivo live imaging. The kinetics of degenerative events such as changes of axonal morphology, axonal transport disruption and intraaxonal calcium homeostasis can be investigated over time using the in vivo live imaging technique. These events can only be examined in a very limited way in fixed tissues and fixation procedure might produce relevant artifacts. Kanamori et al imaged individual RGC axonal bundles in the retina in living animals over time (Kanamori et al., 2010). These axonal bundles were labeled with the dye chloromethyl derivative of fluorescein 
diacetate. They were imaged using confocal scanning laser ophthalmoscopy (CSLO), which is ideal for visualizing retinal nerve fiber layer (RNFL) (Kawaguchi et al., 2006). However, this method does not distinguish individual axons and focused on the retina instead of the optic nerve. Shortly after this, Leung et al developed an imaging model using transgenic mice expressing YFP fluorescent protein under control of a Thy1 promotor (Thy1-YFP16Jrs) (Leung et al., 2011). This method is suitable to differentiate single axons in the retina and optic nerve since less than $1 \%$ of RGCs are labeled in these transgenic mice. Our group established another imaging setup in the living rat in vivo (Koch et al., 2011). In this setup, single RGC axons of the optic nerve are visualized by intravitreal injection of viral vectors expressing fluorophores. The axonal changes can be imaged for up to $8 \mathrm{~h}$ after lesion and can also be re-imaged at later time points after recovery of the rat. Compared to the other methods described above, this imaging setup is best suited to image single RGC axons in the optic nerve. Furthermore, the choice of AAV constructs allows the use of different fluorophores and the co-expression of a fluorophore and a protein or shRNA of interest. For example, a viral vector expressing the fluorophore dsRed was used to image the time course of AAD in the optic nerve (Knöferle et al., 2010) while a viral vector co-expressing the fluorophore dsRed and an shRNA against the protein kinase ROCK was used to evaluate the role of ROCK knockdown on axonal degeneration (Koch et al., 2014). Thus, this imaging setup is a good choice to study the mechanisms of axonal degeneration in the optic nerve.

\subsection{Microfluidic chamber system}

It is necessary to understand the mechanisms of axonal degeneration in order to design therapeutic treatments for neurological diseases (see 1.1 Axonal degeneration). In traditional cell cultures, however, a main difficulty is to specifically identify axons. Recently, the microfluidic chamber system has been increasingly applied in neuroscience research. The chamber system provides a controlled microenvironment for cell culture, separating axonal and soma compartment. It thereby overcomes this limitation of traditional cell culture. Furthermore, this system has a lot of other features and thus can be used to study many 
aspects of axonal degeneration during different pathological conditions. The features of this chamber system as well as its applications are discussed below.

\subsubsection{Features}

The microfluidic chambers are fabricated with polydimethylsiloxane (PDMS). PDMS as a material is suitable for cell culture since it is not toxic and has the features of high gas permeation, low water permeability, thermal stability as well as bio-compatibility (Lee et al., 2004; Merkel et al., 2000; Millet and Gillette, 2012). PDMS is also good for microscopy as it is optically transparent (Millet and Gillette, 2012; Taylor et al., 2003). After PDMS is poured on top of a master and cured by heating, the formed PDMS piece is cut out of the master. The chamber is assembled by placing the PDMS piece on a coverslip. Typically, the chambers are composed of two compartments: one compartment consisting of two holes for loading cells ('soma compartment') and one where the axons will specifically grow into ('axonal compartment') (Taylor et al., 2003). Both compartments are connected by 110 embedded microgrooves which are big enough for neurites to grow through but prevent neuronal cell bodies from passing from one compartment to the other (Taylor et al., 2003) (Figure 1.4).

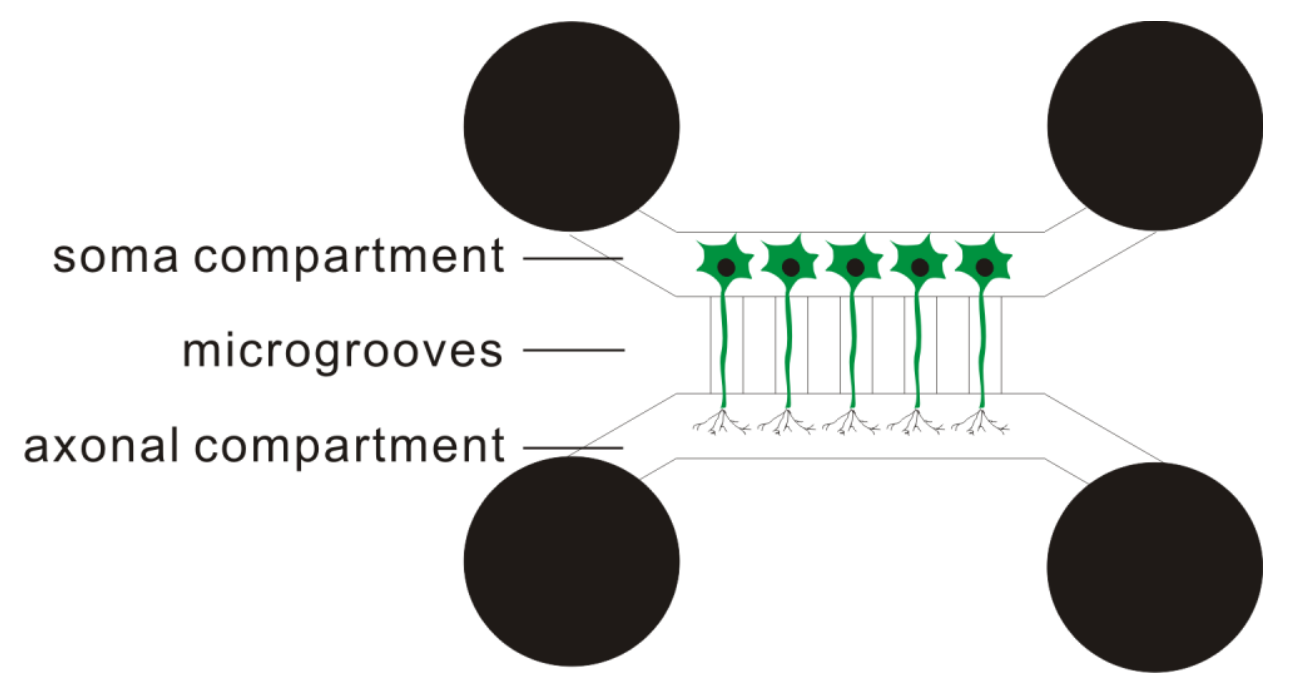

Figure 1.4 Schematic drawing of the microfluidic chamber system.

The microfluidic chamber is composed of two compartments: one compartment consisting of two holes for loading cells ('soma compartment') and one where the axons will specifically grow into ('axonal 
compartment'). Both compartments are connected by embedded microgrooves.

Several kinds of neurons have been successfully cultured in the chambers including embryonic day 18 (E18) cortical neurons, E18 hippocampal neurons, embryonic dorsal root ganglion (DRG) neurons, postnatal day 1 (P1) DRG neurons, P6 DRG neurons, P0 hippocampal neurons and cholinergic neurons (Park et al., 2006). For cultured E18 cortical neurons in the chambers, the axons can be observed in the microgrooves by day in vitro (DIV) 5 (Taylor et al., 2005). By DIV7, the axons have usually crossed the microgrooves and extended into the axonal compartment (Taylor et al., 2005). The isolated axons in the microgrooves stay intact until DIV14 (Taylor et al., 2005).

One main advantage of the chamber system is that axons can be isolated from soma and dendrites (Taylor et al., 2003, 2005). This is based on the fact that axons grow significantly faster and longer than dendrites (Dotti et al., 1988). When the microgrooves are longer than $450 \mu \mathrm{m}$, no dendrites extend to the axonal compartment in cultured cortical neurons until DIV14 (Taylor et al., 2005). This has been confirmed by the use of MAP2 as a dendrite marker (Taylor et al., 2005). When culturing other neuronal cultures, the length of microgrooves can be modulated according to their growth features.

Another main advantage is that soluble insults can be localized specifically to the soma or axonal compartment (Taylor et al., 2003, 2005). The isolated microenvironment can be achieved by applying a volume difference between soma and axonal side. The volume difference produces a small hydrostatic pressure difference. It then leads to a flow only from the higher volume compartment to the lower volume compartment, which acts against the diffusion from the reverse direction. Due to high fluidic resistance of the microgrooves, the fluid flows very slowly. Thus, the soluble insults can be isolated in the smaller volume compartment for up to $20 \mathrm{~h}$ after changing medium.

\subsubsection{Applications}

First, the chamber system can be used to model axonal injury. To model mechanical injury 
of axons, axons are lesioned by vacuum aspiration through the axonal compartment for 5-10 s (Park et al., 2006). The cell side is not affected by the gentle suction due to the high fluidic resistance of the microgrooves (Taylor et al., 2005). To model a chemical lesion, excitotoxins can be applied (Hosie et al., 2012). For example, this excitotoxicity was induced by treatment with the excitatory neurotransmitter glutamate. The chamber allowed to localize glutamate treatment only to the soma or axonal compartment, and to monitor the degenerative changes specifically in the axons.

Second, the chamber system can be used to isolate molecules such as messenger RNA (mRNA) or proteins specifically in the axonal compartment (Taylor et al., 2009). It was shown that some mRNAs were only observed in the axonal but not soma compartment. It was further demonstrated that the mRNAome depends on multifunctional effects. For example, in response to injury, there was a decrease of the mRNAs involved in axonal transport, mitochondria and cytoskeleton. The chamber system thus seems to be suitable for measuring mRNA changes during axonal degeneration.

Third, the chamber system can be used for studying axonal transport of cellular cargos. By using the chamber system, the transport of cargos can be identified specifically in the axons. Anterograde or retrograde transport can also be differentiated easily. In addition, the chamber system is suitable for evaluating the effects of localized treatments on axonal transport. Recently, the chamber system was used to image the transport of quantum dot-labeled nerve growth factor (QD-NGF) in living rat DRG neurons in real time (Cui et al., 2007; Mudrakola et al., 2009). By live imaging, the transport of NGF from the axonal terminal to the cell body could be characterized.

Besides, the chamber system can also be used to screen therapeutic compounds for axonal degeneration or regeneration (Taylor et al., 2005). The CNS axons can be co-cultured with other cells such as oligodendrocytes (Taylor et al., 2005).

\subsection{Aims of this study}

A better understanding of axonal degeneration is crucial for developing neuroprotective 
and pro-regenerative treatments for neurological diseases. However, the underlying molecular mechanisms are only incompletely understood. Several studies demonstrated a transient increase of intraneuronal calcium as the initial crucial event during AAD. However, since calcium influx occurs only transiently within one minute after the lesion, calcium inhibition does not seem to be a promising strategy to treat patients, as a therapeutic treatment will not be available within such a short time after a trauma. In this study, we therefore investigate the molecular mechanisms of AAD following calcium influx and identify promising intervention targets to interfere with AAD.

The first part of the study was to investigate the role of calpain in AAD in the rat optic nerve in vivo. Western blot analysis of optic nerve lysates was performed to investigate whether calpain is activated during AAD. Immunohistochemistry was then carried out to confirm the activation and subcellular localization of activated calpain. Afterwards, in vivo live imaging of the rat optic nerve was used to examine whether pharmacological calpain inhibition interferes with AAD. Finally, several candidate calpain substrates were screened to identify relevant downstream targets of calpain activation. Here, the protein collapsin response mediator protein 2 (CRMP2) was found to be regulated most prominently.

The second part of the study was to evaluate the role of the now-identified calpain target CRMP2 on $A A D$ in vivo and in vitro. An in vitro model for $A A D$ was established in the microfluidic chamber system and the role of CRMP2 overexpression on axonal degeneration was examined in vitro. After that, in vivo live imaging of the rat optic nerve was performed to confirm the neuroprotective effects of CRMP2 overexpression in AAD. Finally, axonal transport of mitochondria was imaged after axotomy in the chamber system in order to investigate the mechanisms on the role of CRMP2 in AAD.

The final part of the study was to characterize CRMP2 interactors as well as further molecular targets, which contribute to AAD. In this work, a proteomics analysis of rat optic nerve lysates was performed to check which proteins are regulated during AAD of the optic nerve. A search tool for the retrieval of interacting genes/proteins (STRING) was used to identify which of the regulated proteins are CRMP2 interactors. 


\section{Materials and Methods}

\subsection{Materials}

\subsubsection{Chemicals and their providers}

Applichem (Darmstadt, Germany): absolute ethanol, dimethyl sulfoxide (DMSO), dithiothreitol (DTT), isopropanol, luminol, methanol, $\mathrm{NaCl}$, non-fat dried milk, paraformaldehyde (PFA), phosphate-buffered saline (PBS), SDS, sucrose, transferrin, tris, triton X-100

Biochrom (Berlin, Germany): fetal calf serum (FCS), trypsin

Biesterfeld (Hamburg, Germany): dow corning 184 A\&B (1.1kg kit)

Braun (Melsungen, Germany): Ringer's solution

Calbiochem (Darmstadt, Germany): calpeptin

Gibco (Darmstadt, Germany): B27 supplement, 10x Hank's balanced salt solution (HBSS), hydrogen peroxide $\left(\mathrm{H}_{2} \mathrm{O}_{2}\right)$, 1x neurobasal medium, $7.5 \%$ sodium bicarbonate solution

Invitrogen (Darmstadt, Germany): MitoTracker Green FM, 1x NuPAGE LDS Sample Buffer, 4-12\% NuPAGE Novex Bis-Tris Minigels

Jackson ImmunoResearch Laboratory Inc (Hamburg, Germany): bovine serum albumin (BSA)

Lonza (Cologne, Germany): Amaxa rat neuron nucleofector kit

PAA cell cuture company (Freiburg, Germany): penicillin/streptomycin solution

Qiagen (Hilden, Germany): GAPDH primer Rn_Gapd_1_SG (NM_017008), QuantiTect reverse transcription kit, QuantiTect SYBR green PCR kit

Roth (Roth, Germany): $30 \%$ acrylamid, ammonium persulfate (APS), tetramethylethylenediamine (TEMED) 
Sigma (Darmstadt, Germany): (p)-coumaric acid, Dulbecco's Modified Eagle Medium (DMEM), 4', 6-diamidino-2-phenylindole (DAPI), ethidium bromide, 45\% D-(+)-glucose solution, laminin, Igepal, mowiol-488, poly-D-lysine (PDL) xylazine, poly-L-ornithine (PLO), trypsine

Tocris (Wiesbaden-Nordenstadt, Germany): ketamine, tianeptine

\subsubsection{Buffers and solutions}

10\% APS: $1 \mathrm{~g}$ APS in $10 \mathrm{~mL} \mathrm{H} \mathrm{H}_{2}$ was aliquoted and stored at $-20^{\circ} \mathrm{C}$.

5\% BSA: $0.5 \mathrm{~g}$ BSA was diluted in $10 \mathrm{~mL} 1 \times$ Tris Buffered Saline with Tween 20 (TBST).

CMF: $450 \mathrm{~mL}$ sterilized $\mathrm{H}_{2} \mathrm{O}, 50 \mathrm{~mL} 10 x$ HBSS, $700-800 \mu \mathrm{L} 7.5 \%$ sodium bicarbonate solution.

25x complete protease inhibitor: 1 tablet was dissolved in $200 \mu \mathrm{L}$ deionized $\mathrm{H}_{2} \mathrm{O}$. Aliquots were kept at $-20^{\circ} \mathrm{C}$ for maximum 12 weeks.

Cortex medium: $48.125 \mathrm{~mL}$ neurobasal medium $\left(4{ }^{\circ} \mathrm{C}\right), 0.25 \mathrm{~mL} 1 \mathrm{mg} / \mathrm{mL}$ transferrin $\left(-20{ }^{\circ} \mathrm{C}\right)$, $0.5 \mathrm{~mL}$ PSN $\left(-20^{\circ} \mathrm{C}\right), 0.125 \mathrm{~mL}$ L-glutamine $\left(-20^{\circ} \mathrm{C}\right), 1 \mathrm{~mL} \mathrm{~B} 27$.

$90 \mathrm{mM}$ coumaric acid: $0.15 \mathrm{~g} \mathrm{p}$-coumaric acid was diluted in $10 \mathrm{~mL}$ DMSO. Aliquots were stored in dark at $-20^{\circ} \mathrm{C}$.

$2.5 \mu \mathrm{g} / \mathrm{mL}$ DAPI: The stock solution was diluted in the filtered PBS and the final dilution contained $0.45 \%$ methanol.

1 M DTT: $0.154 \mathrm{~g}$ DTT dissolved in $1 \mathrm{~mL} \mathrm{H}_{2} \mathrm{O}$ was aliquoted and stored at $-20^{\circ} \mathrm{C}$.

Enhanced chemiluminescence (ECL) Reagent 1: $100 \mu \mathrm{L} 250 \mathrm{mM}$ luminol $\left(-20^{\circ} \mathrm{C}\right), 44 \mu \mathrm{L}$ $90 \mathrm{mM}$ p-coumaric acid $\left(-20^{\circ} \mathrm{C}\right), 1 \mathrm{~mL} 1 \mathrm{M} \mathrm{PH} 8.5$ Tris, $8.85 \mathrm{~mL} \mathrm{H} \mathrm{O}_{2}$.

ECL Reagent 2: $6 \mu \mathrm{L} 30 \% \mathrm{H}_{2} \mathrm{O}_{2}\left(4^{\circ} \mathrm{C}\right), 1 \mathrm{~mL} 1 \mathrm{M}$ PH 8.5 Tris, $9 \mathrm{~mL} \mathrm{H}_{2} \mathrm{O}$.

10x electrophoresis buffer: 250 mM Tris (30.24 g), $1.9 \mathrm{M}$ glycine (142.5 g) and 1\% SDS (10 g) were dissolved in $1 \mathrm{~L} \mathrm{H}_{2} \mathrm{O}$. 
Hek293 cells medium: $500 \mathrm{~mL}$ DMEM, $45 \mathrm{~mL} \mathrm{FCS}$, and $5.5 \mathrm{~mL}$ penicillin/streptomycin solution.

HK $\mu \mathrm{E}$ buffer: $10 \mathrm{mM}$ Hepes (1.19 g) adjusted to $\mathrm{pH} 7.2,142 \mathrm{mM} \mathrm{KCl}(5.3 \mathrm{~g}), 5 \mathrm{mM}$ $\mathrm{MgCl}_{2} .6 \mathrm{H}_{2} \mathrm{O}(0.51 \mathrm{~g})$ and $1 \mathrm{mM} \mathrm{EGTA}(0.19 \mathrm{~g})$ were dissolved in $1 \mathrm{~L}_{2} \mathrm{O}$.

5x Laemmli buffer: $0.756 \mathrm{~g}$ tris in $5 \mathrm{~mL} \mathrm{H} \mathrm{H}_{2} \mathrm{O}$ adjusted to $\mathrm{pH} 6.8,2 \mathrm{~g}$ SDS, $10 \mathrm{~mL}$ glycerin, $0.001 \mathrm{~g}$ bromophenol blue, $308 \mathrm{mg}$ DTT.

$250 \mathrm{mM}$ luminol: $0.44 \mathrm{~g}$ luminol (3-aminophthalydrazide) was diluted in $10 \mathrm{~mL}$ DMSO. Aliquots were stored in dark at $-20^{\circ} \mathrm{C}$.

Lysis buffer: $1 \mathrm{~mL} \mathrm{HK \mu E}$ buffer, $10 \mu \mathrm{L}$ Igepal, $40 \mu \mathrm{L}$ complete protease inhibitor (25x), $50 \mu \mathrm{L}$ phosSTOP-phosphatase inhibitor (20x), $1 \mu \mathrm{L} 1 \mathrm{M}$ DTT.

Mowiol: $5 \mathrm{~g}$ mowiol were mixed in $20 \mathrm{~mL}$ PBS for $1 \mathrm{~h}$ at $40{ }^{\circ} \mathrm{C}$, and then mixed with $10 \mathrm{~mL}$ glycerol for $1 \mathrm{~h}$ to overnight under constant stirring. After centrifugation at $5000 \mathrm{rpm}$ for $15 \mathrm{~min}$, the collected supernatant was regulated to $\mathrm{pH} 8.0$. After adding $0.3 \mathrm{~mL} 2 \%$ sodium azide and about $9 \mathrm{mg} \mathrm{n}$-propyl gallate, the solution was centrifuged at $4000 \mathrm{rpm}$ for $20 \mathrm{~min}$ to remove the bubbles. Aliquots were kept at $-20^{\circ} \mathrm{C}$.

PBS: $9.55 \mathrm{~g}$ PBS powder were diluted in $1000 \mathrm{~mL} \mathrm{H} \mathrm{H}_{2} \mathrm{O}$ and then filtered.

$0.1 \mathrm{mg} / \mathrm{mL}$ PDL: $5 \mathrm{mg}$ PDL were dissolved in $50 \mathrm{~mL}$ sterilized $\mathrm{H}_{2} \mathrm{O}$ and then filtered. Aliquots were kept at $-20^{\circ} \mathrm{C}$.

4\% PFA: $20 \mathrm{~g}$ PFA were dissolved in $300 \mathrm{~mL}$ PBS at $55^{\circ} \mathrm{C}$. After adding one pellet of $\mathrm{NaOH}$, the solution was filtered, cooled to $4{ }^{\circ} \mathrm{C}$ and adjusted to $\mathrm{pH} 7.4$ at $4{ }^{\circ} \mathrm{C}$. More PBS was filled up to $500 \mathrm{~mL}$ of solution in total, and the prepared solution was kept at $4{ }^{\circ} \mathrm{C}$.

20x phosSTOP-phosphatase inhibitor: 2 tablets were diluted in $1 \mathrm{~mL}$ deionized $\mathrm{H}_{2} \mathrm{O}$. Aliquots were kept at $-20^{\circ} \mathrm{C}$ for maximum 6 months.

$1 \mathrm{mg} / \mathrm{ml} \mathrm{PLO}$ : $50 \mathrm{mg}$ PLO were diluted in $50 \mathrm{ml}$ borate buffer, sterilized by filtration, and kept at $4{ }^{\circ} \mathrm{C}$. 
10\% separating gel: $3.545 \mathrm{~mL} \mathrm{H} \mathrm{H}_{2} \mathrm{O} 2.125 \mathrm{~mL} 4 \times$ tris $\mathrm{pH} 8.8,2.83 \mathrm{~mL} 30 \%$ acrylamide, $42.5 \mu \mathrm{L}$ 10\% APS, $4.25 \mu \mathrm{L}$ TEMED.

Stacking gel: $3.05 \mathrm{~mL} \mathrm{H} \mathrm{H}_{2} \mathrm{O}, 1.25 \mathrm{~mL} 4 \mathrm{x}$ tris $\mathrm{pH} 6.8,0.65 \mathrm{~mL} 30 \%$ acrylamide, $25 \mu \mathrm{L} 10 \%$ APS, $5 \mu \mathrm{L}$ TEMED.

1x TBST: 10x TBST was diluted 10 times in $\mathrm{H}_{2} \mathrm{O}$. $\mathrm{pH}$ adjustment was not needed.

10x TBST : $24.2 \mathrm{~g}$ tris, $80 \mathrm{~g} \mathrm{NaCl}$, and $10 \mathrm{~mL}$ tween 20 were dissolved in $\mathrm{H}_{2} \mathrm{O}$ up to $1 \mathrm{~L}$ solution, and adjusted to $\mathrm{pH} 7.6$.

1x transfer buffer: $2.7 \mathrm{~g}$ tris, $12.96 \mathrm{~g}$ glycine, $180 \mathrm{~mL}$ methanol, $720 \mathrm{~mL} \mathrm{H}_{2} \mathrm{O}$.

10\% triton X-100: $900 \mu \mathrm{L}$ 0.05M Tris/1.5\% NaCl, $100 \mu \mathrm{L}$ triton X-100.

0.05M tris/1.5\% NaCl: $6.06 \mathrm{~g}$ tris, $15 \mathrm{~g} \mathrm{NaCl}, 1000 \mathrm{~mL} \mathrm{H} \mathrm{H}_{2} \mathrm{O}$.

25000 units/mL trypsin: 25000 units trypsin were diluted in $1 \mathrm{~mL} \mathrm{CMF}$, adjusted to $\mathrm{pH} 7.6$, and sterilized by filtration. Aliquots were kept at $-20^{\circ} \mathrm{C}$.

\subsubsection{Plasmids}

p.Bluescript (Stratagene, La Jolla, USA)

p.CMV-CRMP2-flag (generous gift from Mahnaz Moradi-Améli, Université Lyon 1, France)

p.CMV-EGFP (generous gift from Uwe Michel, University of Göttingen, Germany)

p.AAV.hSyn-CRMP2-hSyn-mcherry (generous gift from Uwe Michel, University of Göttingen, Germany)

p.AAV.hSyn-mcherry-hSyn (generous gift from Uwe Michel, University of Göttingen, Germany)

p.AAV.hSyn-EGFP (generous gift from Jan C. Koch, University of Göttingen, Germany)

p.AAV.hSyn-mito-RFP (generous gift from Zara d'Hedouville, University of Göttingen, Germany)

p.TurboRFP-mito (Evrogen, Heidelberg , Germany)

p.EGFP-N2 (Clontech, Heidelberg, Germany) 


\subsubsection{Animals}

Adult female Wistar rats (Charles River, Sulzfeld, Germany)

Embryonic day 18 Wistar rats (Central animal facility of University Medicine Göttingen, Germany)

\subsubsection{Equipment}

Analytical reversed phase-C18 column (Dr. Maisch, Ammerbuch-Entringen, Germany) Autoclave (Systec, Linden, Germany)

Axioplan 2 microscope equipped with AxioCam HRm camera (Carl Zeiss Microimaging, Göttingen, Germany)

Axiovert $200 \mathrm{M}$ inverted microscope (Carl Zeiss Microimaging, Göttingen, Germany)

Balance (Sartorius, Göttingen, Germany)

C1000 Touch thermal cycler (Bio-Rad, München, Germany)

Cell culture hood (Heraeus, Hanau, Germany)

Centrifuge 5810R (Eppendorf, Wesseling-Berzdorf, Germany)

Centrifuge 5418R (Eppendorf, Wesseling-Berzdorf, Germany)

Cover slides 24 x 60mm (Menzel, Braunschweig, Germany)

Cryomatrix (Thermo Scientific, Bremen, Germany)

Cryostat, CM 3050 S (Leica, Mannheim, Germany)

CTI-Controller 3700 (Carl Zeiss Microimaging, Göttingen, Germany)

Curix 60 Developer (Agfa, Koln, Germany)

Driller (Proxxon, Föhre, Germany)

Drying Oven (Thermo Scientific, Bremen, Germany)

Electrophoresis chamber (BioRad, München, Germany)

Electrophoresis power supply (BioRad, München, Germany)

Forceps (Fine Science Tools, Heidelberg, Germany)

Hamilton syringe (Hamilton, Planegg, Germany)

Heracell 150i $\mathrm{CO}_{2}$ incubator (Thermo Scientific, Bremen, Germany)

Ice machine (Scotman, Pogliano Milanese, Italy) 
Mass spectrometer (AB SCIEX, Darmstadt, Germany)

MR 3000 Shaker (Heidolph, Schwabach, Germany)

Nanodrop spectrophotometer (Thermo Scientific, Bremen, Germany)

Nitrocellulose transfer membrane (Applichem, Darmstadt, Germany)

Nucleofector Device (Lonza, Cologne, Germany)

Petri dish (Sarstedt, Nümbrecht, Germany)

pH Meter (Sartorius, Göttingen, Germany)

Pipettes (Eppendorf, Wesseling-Berzdorf, Germany)

10-0 polyamide suture (needle $3 / 8$ circle) (Ethicon, Norderstedt, Germany)

Protein ladder (BioRad, München, Germany)

Pseudo-confocal ApoTome device (Carl Zeiss Microimaging, Göttingen, Germany)

Pulsoximeter (Nonin Braunfels, Germany)

Scalpel, size 10 (Bard-Parker, Singen, Germany)

Scissors (Fine Science Tools, Heidelberg, Germany)

Self-packed reversed phase-C18 precolumn (Dr. Maisch, Dr. Maisch, Ammerbuch-Entringen, Germany)

Spacer plates (Biorad, München, Germany)

Tempcontrol 37-2 (Carl Zeiss Microimaging, Göttingen, Germany)

Thermocoagulator (Fine Science Tools, Heidelberg, Germany)

Thermomixter (Eppendorf, Wesseling-Berzdorf, Germany)

40x Water immersion objective (Carl Zeiss Microimaging, Göttingen, Germany)

6-well culture plate (Sarstedt, Numbrecht, Germany)

12-well culture plate (Sarstedt, Numbrecht, Germany)

24-well culture plate (Sarstedt, Numbrecht, Germany)

48-well culture plate (Costar, Wiesbaden, Germany)

Warming pad for rats (Kent Scientific, Connecticut, USA)

Western blot Electrophoresis chambers (BioRad, München, Germany)

Whatman gel blotting paper (GE Healthcare, Chalfont St. Gilles, UK) 


\subsubsection{Antibodies}

\subsubsection{Primary antibodies}

Mouse anti-Dynein IC (monoclonal, MMS-400P, Covance, München, Germany)

Mouse anti-Flag M2 antibody (monoclonal, F1804, Sigma, Darmstadt, Germany)

Mouse anti-GAPDH (monoclonal, G9545, Biotrend, Köln, Germany)

Mouse anti-Map-2 (monoclonal, MAB3418, Millipore, Darmstadt, Germany)

Mouse anti-Smi31 (monoclonal, SMI-31R, Covance, München, Germany)

Mouse anti-spectrin (monoclonal, BML-FG6090, Enzo, Ansbach, Germany),

Mouse anti-Tau (monoclonal, T9450, Sigma, Darmstadt, Germany)

Rabbit anti-Atg5 (polyclonal, AP1812b, Abgent, Hamburg, Germany)

Rabbit anti-Beclin-1 (polyclonal, \#3738, Cell signaling, Cambridge, UK),

Rabbit anti-CRMP2 (polyclonal, \#9393, Cell signaling, Cambridge, UK),

Rabbit anti-cleaved spectrin (a generous gift from $\mathrm{Dr}$ Robert Siman, University of Pennsylvania, USA)

\subsubsection{Secondary antibodies}

Donkey anti-mouse cy2 secondary antibody (polyclonal, 715-226-150, Dianova, Hamburg, Germany)

Donkey anti-rabbit cy3 secondary antibody (monoclonal, 711-165-152, Dianova, Hamburg, Germany)

Goat anti-mouse cy3 secondary antibody (polyclonal, 115-165-146, Dianova, Hamburg, Germany)

Goat anti-mouse horseradish peroxidase (HRP) (7076P2, Cell signaling, Cambridge, UK) Goat anti-rabbit HRP (7074P2, Cell signaling, Cambridge, UK)

\subsubsection{Software}

SPSS 16.0 (IBM, USA)

Image J 1.49a (NIH, USA) 
AxioVision 4.8 (Zeiss Carl Zeiss Microimaging, Göttingen, Germany)

ProteinPilot 5.0 software rev4769 (AB SCIEX, Germany).

Scaffold software version 4.4.1.1 (Proteome Software Inc., Portland)

Bio-Rad CFX manager 3.0 software (Bio-Rad, Germany)

CoreIDRAW X3 software (Corel Corporation, Canada)

\subsection{Methods}

\subsubsection{Plasmids}

The following plasmids were used to check the role of CRMP2 overexpression on axonal degeneration in vitro: p.CMV-CRMP2-flag (Rogemond et al., 2008), p.CMV-EGFP (Genbank ID: KT343252). The plasmids used for adeno-associated virus (AAV) production are: p.AAV.hSyn-CRMP2-hSyn-mcherry (Genbank ID: KT345944), p.AAV.hSyn-mcherry-hSyn (Genbank ID: KT345943), p.AAV.hSyn-mito-RFP (Genebank ID: KT358727). Both p.AAV.hSyn-CRMP2-hSyn-mcherry and p.AAV.hSyn-mcherry-hSyn contain two human synapsin promoters.

p.AAV.hSyn-mito-RFP was cloned with the following procedures: The insert containing the red fluorescent protein (RFP) and a mitochondrial targeting sequence (MTS) was cut from p.TurboRFP-mito. The resulting insert was then subcloned into pBS-Shuttle at the Nhel and Notl restriction sites. The insert containing RFP and MTS cut from the shuttle vector with a Nhel and Sall digestion was then ligated into p.EGFP-N2. Afterwards, the insert containing MTS and RFP was cut from pEGFP-N2 with Nhel and Acc65I digestion, and it was subcloned to p.AAV.hSyn-EGFP (Koch et al., 2011). After a digestion with BstAPI, the backbone was re-ligated. Finally, the resulting plasmid p.AAV-hSyn-TurboRFP-mito was sequenced and used for AAV production.

For cloning of p.AAV.hSyn-CRMP2-hSyn-mcherry, the CRMP2 fragment from p.CMV-CRMP2-flag was cut with EcoRV and HindlII. It was then ligated into a Hincll- and HindIII- cut pBluescript to get the resulting plasmid pBSKII-CRMP2. After pBSKII-CRMP2 was cut with Xmal and Sacl, the resulting insert was ligated into an Agel- and Sacl- cut pAAV 
vector in $5^{\prime}$ of a bGH polyA tail and $3^{\prime}$ of an hSyn promoter. The resulting plasmid pAAV-CRMP2-hSyn-mcherry was sequenced and used for AAV production.

\subsubsection{Adeno-associated virus}

To check the role of calpain inhibitor calpeptin on $A A D$ using in vivo live imaging, AAV.hSyn-EGFP virus was produced to visualize RGC axons as previously described (Koch et al., 2011). To image the kinetics of mitochondrial transport after axotomy in vitro, AAV.hSyn-RFP-mito virus was produced to label mitochondria. To check the role of CRMP2 on mitochondrial transport in vitro and on AAD in vivo, AAV.hSyn-CRMP2-hSyn-mcherry virus was produced to visualize the axons overexpressing CRMP2. AAV.hSyn-mcherry-hSyn virus was produced as control. All these viral vectors were provided by Uwe Michel and Jan C. Koch (University of Göttingen, Germany).

For all experiments, AAV of the pseudotype $1 / 2$ were used. To generate this pseudotype of AAV, AAV2 inverted terminal repeats (ITR) were packed into AAV1/AAV2 hybrid capsids in a molar ratio of 1:1. To produce both AAV.hSyn-CRMP2-hSyn-mcherry virus and AAV.hSyn-mcherry-hSyn virus, the pACG-2 helper-plasmid (kind gift of Arun Srivastava, University of Florida, USA) was used, which results in the hybrid serotype AAV1/mutAAV2 (Zhong et al., 2008). Viral vectors were produced as described before (Koch et al., 2011). Briefly, HEK293 cells were transfected with calcium phosphate and a plasmid mixture. This plasmid mixture consisted of the respective pHELPER, pAAV-expression vector, $\mathrm{pH} 21$ and pAAV-RC (molar ratio 1:1:0.5:0.5). Both plasmids pAAV-RC and pHELPER were from Stratagene. The plasmid pH21 (pAAV1) expressing AAV serotype 1 capsids, was a kind gift of Helen Fitzsimons (Neurologix, Inc. OSU Comprehensive Cancer Center, Columbus, USA) and Matthew During (Molecular Virology, Immunology, and Medical Genetics, Columbus, USA). At $48 \mathrm{~h}$ after transfection, the cells were harvested. Viral vectors were purified by dialysis and virus gradient centrifugation in iodixanol. To obtain high titer viral stocks, fast protein liquid chromatography was performed. To determine viral titers, the GPCR analysis with appropriate plasmid standards was performed. Toxicity and transduction rates of all the viral vectors were tested in the primary cortical neurons in vitro and after intravitreal injections in 
vivo.

\subsubsection{Surgical procedures}

All animal experiments were performed with regards to the regulations of the local animal research council and legislation of the State of Lower Saxony (Germany). In all the experiments, adult female Wistar rats (250-350 g) were used. For anesthesia, rats were injected intraperitoneally with $10 \%$ ketamine ( $95 \mathrm{mg} / \mathrm{kg}$ body weight) and $2 \%$ xylazine ( 7 $\mathrm{mg} / \mathrm{kg}$ body weight).

\subsubsection{Intravitreal injections}

For calpain inhibition, $10 \mathrm{mM}$ calpeptin in 7\% DMSO or 7\% DMSO in deionized $\mathrm{H}_{2} \mathrm{O}$ as control was intravitreally injected $2.5 \mathrm{~h}$ before ONC. A total volume of $3 \mu \mathrm{L}$ was injected per eye. For imaging the time course of $A A D$ after calpain inhibition, $1.7 \times 10^{8}$ transforming units (TU) AAV.hSyn-EGFP per eye were intravitreally injected 2 weeks before imaging. To confirm CRMP2 overexpression by AAV.hSyn-CRMP2-hSyn-mcherry in vivo, $2.8 \times 10^{8}$ TU AAV.hSyn-mcherry-hSyn or $2.1 \times 10^{8}$ TU AAV.hSyn-CRMP2-hSyn-mcherry per eye were intravitreally injected 4 weeks before dissecting optic nerves and retinas. For imaging the time course of AAD after CRMP2 overexpression in vivo, $2.8 \times 10^{8}$ TU AAV.hSyn-mcherry-hSyn or $2.1 \times 10^{8}$ TU AAV.hSyn-CRMP2-hSyn-mcherry per eye were injected 4 weeks before imaging. The total volume of injected viral vectors was $5 \mu \mathrm{L}$ per eye. The titers of AAV were previously optimized according to equal transduction rates of the retina with no obvious toxicity.

The procedures of intravitreal injections with a Hamilton syringe were performed as described previously (Koch et al., 2011). The anesthetized rat was placed on the surgery table under a Carl Zeiss operating stereomicroscope. While the eye bulb was fixed with the thumb and index finger of the left hand, the syringe was inserted behind the inferior temporal limbus and towards the upper nasal side with the right hand. The needle was pushed carefully until its tip was above the upper nasal quadrant of the retina. The substances were 
then slowly injected over $1 \mathrm{~min}$. After waiting for another $1 \mathrm{~min}$, the needle was pulled out of the eye slowly. Eye ointment (Bepanthen ${ }^{\circledR}$ ) was applied on both eyes. After AAV injection, the rat was placed on a warming pad until it woke up. After DMSO or calpeptin injection, the rat was placed on a warming pad for further surgery.

\subsubsection{Optic nerve crush}

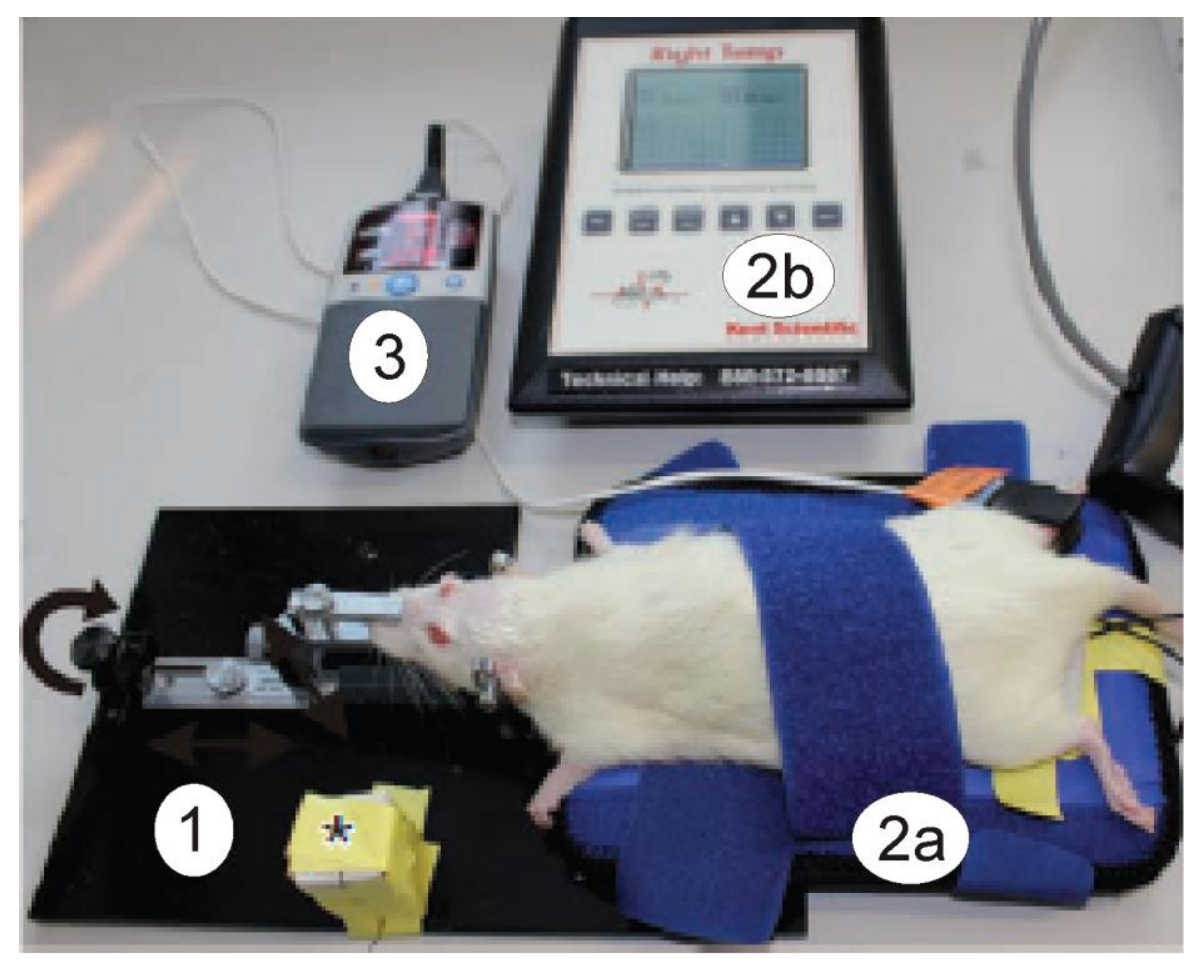

Figure 2.1 Optic nerve surgery setup.

During the surgery of optic nerve, the rat was fixed in the rat positioning setup (1). The body of the rat was placed on a warming pad (2a), which was controlled by a thermocontrol system (2b). A pulsoximeter (3) was used to monitor heart rate and blood oxygen saturation of the rat (modified from Koch et al., 2011).

Surgery of the optic nerve was performed as described before (Koch et al., 2011). The anesthetized rat was placed on a warming pad under a Carl Zeiss surgical microscope. A pulsoximeter was used to monitor heart rate and blood oxygen saturation of the rat. After 
clipping the fur on its head, the rat was fixed in the rat positioning setup and tilted at an angle of $\sim 30^{\circ}$ to its right side (Figure 2.1). The skin in the operating area was disinfected with $70 \%$ ethanol, and a median incision was performed using a scalpel. Afterwards, the operating field was opened with custom-made hooks. A thermocoagulator was used to transect the connective tissue along the orbital rim. After cutting layer by layer, the intraorbital gland became visible. While the gland was covered by small gauze swabs, the protruding bony parts of the orbital rim were removed carefully by a small driller. After washing away the bone chips with warm Ringer's solution, the supraorbital nerve and vein on top of the gland were transected by the thermocoagulator. After the gland was moved to the front, the eye was rotated by pulling the underlying superior rectus muscle carefully to the lateral side of the rat. Another hook was used to fix the eye in this position. The fat and connecting tissue on top of the optic nerve were removed, and this was followed by a longitudinal incision of the dura surrounding the optic nerve. Care was taken not to damage the blood vessels. The optic nerve was finally exposed by retracting the dura to both sides of the incision. For optic nerve crush, a 10-0 polyamide suture was tied tightly around the optic nerve for $30 \mathrm{~s}$.

\subsubsection{Primary cortical neuron culture}

\subsubsection{Fabrication of microfluidic chambers}

Microfluidic chambers were produced as described elsewhere (Park et al., 2006; Rhee et al., 2005). The chambers contain four wells and two main channels. The two channels are connected with 110 embedded microgrooves (7 $\mu \mathrm{m}$ wide, $3 \mu \mathrm{m}$ high and $450 \mu \mathrm{m}$ long). To produce the chambers, the master molds were prepared by Sarah Köster (University of Göttingen, Germany). The PDMS prepolymer and the catalyst were weighted out in a ratio of 10:1. About $6 \mathrm{~g}$ of the mixture were used for each chamber. The two components were mixed thoroughly in a disposable cup until the bubbles distributed homogenously. The mix was then poured over the master mold in a petri dish. Afterwards, the petri dish was placed in a leveled laboratory oven at $60{ }^{\circ} \mathrm{C}$ for $90 \mathrm{~min}$ until the PDMS mixture was solidified and became transparent. The cured PDMS piece was carefully separated from the master mold 
using a sharp blade. The reservoirs were punched, and the PDMS piece was trimmed. When handling it, care was taken not to touch the imprinted side. To reuse the chambers, they were first rinsed with deionized $\mathrm{H}_{2} \mathrm{O}$ for at least 10 times. They were then placed in a clean beaker with deionized $\mathrm{H}_{2} \mathrm{O}$ at $37{ }^{\circ} \mathrm{C}$ overnight. After further washing with deionized $\mathrm{H}_{2} \mathrm{O}$, they were left for drying. Each chamber can be reused for 10 times. Finally, they were stored in the clean petri dishes sealed with parafilm. The imprinted side of chambers was placed upside.

\subsubsection{Coating of culture plates and coverslips}

12-well and 24-well culture plates were coated with PLO and laminin. PLO was diluted 10 times with sterilized $\mathrm{H}_{2} \mathrm{O}$. 12-well or 24-well plates were incubated with $1 \mathrm{~mL}$ or $0.5 \mathrm{~mL}$ diluted PLO per well at room temperature (RT) for at least $4 \mathrm{~h}$. The plate with PLO was then rinsed with sterilized $\mathrm{H}_{2} \mathrm{O}$ for 2 times. Next, laminin was diluted with sterilized $\mathrm{H}_{2} \mathrm{O}$ in a ratio of 1:1000. The 12 -well or 24 -well plate was incubated with $1 \mathrm{~mL}$ or $0.5 \mathrm{~mL}$ diluted laminin per well in the incubator overnight, and then rinsed with neurobasal medium 2 times. Finally, the coated plate was filled with $1 \mathrm{~mL}$ cortex medium, and kept in the incubator until seeding of the cells.

For the scratch assay and microfluidic chamber experiments, cortical neurons were cultured onto the coverslips in 48-well culture plates and/or in microfluidic chambers. To clean the coverslips, they were first sonicated in deionized $\mathrm{H}_{2} \mathrm{O}$ at $60^{\circ} \mathrm{C}$ for $30 \mathrm{~min}$. Coverslips were further cleaned by shaking in acetone for $5 \mathrm{~min}$ and in 100\% ethanol for $5 \mathrm{~min}$. Finally, they were rinsed with deionized $\mathrm{H}_{2} \mathrm{O}$ and left to dry completely. They were kept in a clean culture dish and sealed with parafilm for later use. For sterilization, they were placed in $100 \%$ ethanol for a few seconds and then flamed. All the following procedures were performed in a cell culture hood. For coating, the sterilized coverslips were covered with $0.1 \mathrm{mg} / \mathrm{mL} P D L$ at $37{ }^{\circ} \mathrm{C}$ for $30 \mathrm{~min}$. After washing with sterilized $\mathrm{H}_{2} \mathrm{O}$ for 2 times, they were left to dry completely in the hood. After adding cortex medium, the plates or chambers with coverslips were kept in the incubator before seeding. 


\subsubsection{Assembling of microfluidic chambers}

Any remaining structures or dirt on the surface of the chambers were removed by a scotch tape. All the following procedures were performed in a cell culture hood. For sterilization, the chambers were immersed in $70 \%$ ethanol in petri dishes. After shaking the chambers by hand for $5 \mathrm{~min}$, the ethanol was removed by pump suction. The chambers were then left to dry in the culture hood for at least $3 \mathrm{~h}$. Afterwards, the sterilized chambers were placed on coated glass coverslips with the imprinted side downward. After gently pressing the chambers, they were sealed to the coverslips. $200 \mu \mathrm{l}$ pre-warmed cortex medium were immediately added in one well of each chamber. The medium was distributed to the other well through the main channel. The side with cortex medium was marked as soma side, while the other side was the axonal side accordingly. After the medium had gone across the microgrooves, warm medium was added to the axonal side of the chambers. Finally, the assembled chambers were kept in the incubator until the cortical neurons were prepared.

\subsubsection{Primary cortical neuron culture}

The preparation of primary rat cortical neurons was performed as described previously (Ivins et al., 1998). E18 rats were sacrificed with $\mathrm{CO}_{2}$ insufflation and fixed on a preparation table. After disinfecting the front side of the rat with $70 \%$ ethanol, the embryos were taken out and put in ice-cold calcium magnesium-free (CMF) buffer. The heads of the embryos were separated and put in another dish with CMF. The cortices were dissected in the lid of a petri dish under the microscope, and collected in a $10 \mathrm{~mL}$ conical tube ewith ice cold CMF. After centrifugation at $800 \mathrm{rpm}$ for $4 \mathrm{~min}$ at $4{ }^{\circ} \mathrm{C}$, the supernatant was replaced with $1 \mathrm{~mL}$ ice-cold trypsin $(25000$ units $/ \mathrm{mL})$. After incubation in a $37{ }^{\circ} \mathrm{C}$ water bath for $10 \mathrm{~min}, 50 \mu \mathrm{L}$ ice-cold DNAse $(5 \mathrm{mg} / \mathrm{mL}$ ) was added to dissolve DNA-aggregates released from damaged cells. After spinning the tube at $800 \mathrm{rpm}$ for $1 \mathrm{~min}$, the trypsin was immediately removed and the pellet was covered with $1 \mathrm{~mL}$ FCS. The tissues were triturated by gently pipetting up and down with a $1000 \mu \mathrm{L}$ pipette tip for 3 times. After spinning it down, the supernatant with the suspended cells was collected into another $10 \mathrm{~mL}$ conical tube. The pellet was further triturated in $1 \mathrm{~mL}$ fresh cortex medium with a fire-polished Pasteur pipette for 3 times gently. 
The supernatant was again collected. After the collected supernatant with re-suspended cells was centrifuged for $4 \mathrm{~min}$, the supernatant was removed and the cells were re-suspended in warm cortex medium. For counting the cells, the coverslip was adhered to the hemocytometer via suction. The diluted cells with PBS were mixed with trypan blue. $10 \mu \mathrm{L}$ of the mixture was pipetted to each side of a hemocytometer. Afterwards, the cells were seeded in the culture plates or microfluidic chambers. For transduced cells, $3 \times 10^{5}$ and $1.5 \times$ $10^{5}$ cells were loaded in each well of 24 -well and 48-well plates respectively. $1.5 \times 10^{5}$ cells were seeded per chamber. For transfected cells, $1 \times 10^{6}$ and $3 \times 10^{5}$ transfected cells were seeded in each well of 12-well culture plates and each microfluidic chamber respectively. The cells were then cultured in the incubator at $37^{\circ} \mathrm{C}$ with $5 \% \mathrm{CO}_{2}$ and $95 \%$ humidity. Half of the culture medium was changed in the culture plates every 3 days.

\subsubsection{Nucleofection}

To overexpress CRMP2 by p.CMV-CRMP2-flag, cortical neurons were transfected with $4 \mu \mathrm{g}$ p.CMV-EGFP alone or co-transfected with both $2.5 \mu \mathrm{g}$ p.CMV-CRMP2-flag and $1.5 \mu \mathrm{g}$ p.CMV-EGFP. To confirm CRMP2 overexperssion by p.AAV.hSyn-CRMP2-hSyn-mcherry, cortical neurons were transfected with 4 Mg pAAV.hSyn-CRMP2-hSyn-mcherry or pAAV.hSynmcherry-hSyn. The transfection was carried out using the Amaxa rat neuron nucleofector kit according to the manufacturer's instructions. For each sample, $4 \mu \mathrm{g}$ plasmid DNA was diluted with $\mathrm{H}_{2} \mathrm{O}$ up to $10 \mu \mathrm{L} .2 \times 10^{6}$ cells per sample were centrifuged at $1 \times 10^{3} \mathrm{rpm}$ for $1.5 \mathrm{~min}$ at RT. The nucleofector solution was prepared and pre-warmed at RT. After removing the supernatant carefully, the cell pellet was re-suspended in the mixture of $90 \mu \mathrm{L}$ nucleofector solution and $10 \mu \mathrm{L}$ diluted plasmid. The cell/DNA suspension was transferred to the cuvette. Program 0-05 was applied for the inserted cuvette in the nucleofector device. As soon as the program was finished, the sample was transferred to the pre-warmed mixture of $350 \mu \mathrm{L}$ cortex medium and $50 \mu \mathrm{L}$ FCS. The cells were seeded immediately after finishing the procedures for all samples. $1 \times 10^{6}$ transfected cells per well were seeded in 12-well culture plates. At $3 \mathrm{~h}$ after seeding, the medium was changed completely. 


\subsubsection{Viral transduction}

Viral transduction was performed 2-3 hours after seeding. The titers of the viral vectors were previously optimized resulting in equal transduction rates (90\% of all cells) and only minor toxicity. In the microfluidic chambers, $100 \mu \mathrm{L}$ medium mixed with the viral vector was added in both wells of the soma side per chamber. In each chamber, $1.5 \times 10^{5}$ cells were transduced with $1 \times 10^{7}$ transforming units (TU) AAV.mito-RFP, $5 \times 10^{6}$ TU AAV.hSynCRMP2-hSyn-mcherry or $1 \times 10^{6}$ TU AAV.hSyn-mcherry-hSyn. $24 \mathrm{~h}$ after seeding, half of the medium was exchanged. In the following days, half of the medium was changed every two days. In the 24-well plates, the medium per well was reduced to $300 \mu \mathrm{L}$ just before adding viral vectors. Afterwards, $50 \mu \mathrm{L}$ medium mixed with viral vector were added per well. In each well, $3 \times 10^{5}$ cells were transduced with $1 \times 10^{7}$ TU AAV.hSyn-CRMP2-hSyn-mcherry or 2 $x 10^{6}$ TU AAV.hSyn-mcherry-hSyn. $24 \mathrm{~h}$ after transduction, $150 \mu \mathrm{L}$ fresh medium were added back to each well. Afterwards, half of the medium was changed every 2 days.

\subsubsection{Cell seeding and culturing in microfluidic chambers}

The medium in the previously prepared microfluidic chambers was exchanged against fresh medium completely. As soon as the cortical neurons were prepared, the medium on the soma side was aspirated. The cells were gently pipetted at the entrance of the main channel on the soma side, and this channel was named 'soma compartment'. The other main channel was called 'axonal compartment'. For nucleofection experiments, $3 \times 10^{5}$ previously transfected cells were seeded per chamber in $75 \mu \mathrm{L}$ medium. At $3 \mathrm{~h}$ after seeding, the medium in the chambers was completely substituted by fresh medium. For viral transduction experiments, $1.5 \times 10^{5}$ cells in $75 \mu \mathrm{L}$ medium were seeded in each chamber. At 2 to 3 hours after seeding, $100 \mu \mathrm{L}$ medium mixed with the viral vector were added in the wells of the soma side in each chamber. In both cases, half of the medium was changed $24 \mathrm{~h}$ after seeding. In the following days, half of the medium was changed every 2 days. On DIV8, axons extended across the microgrooves and into the axonal compartment. 


\subsubsection{Axotomy in microfluidic chamber}

On DIV8, an axotomy was performed by a glass Pasteur pipette connected to vacuum. When the pipette was placed close to the entrance of the axonal compartment for $3-5 \mathrm{~s}$, the medium was emptied from the wells of the axonal side. The axons were lesioned by the passing of a resulting bubble through the axonal compartment. Immediately, new medium was added to one empty well. The medium flowed into the other empty well re-filling the axonal compartment. Cells on the soma side were not damaged by the gentle suction due to the high fluidic resistance of the microgrooves. To confirm the axotomy, the chamber was checked under the microscope. In the case that the axons were not sufficiently lesioned, the above procedures needed to be repeated once.

\subsubsection{Scratch assay}

Before seeding, the medium was exchanged against $175 \mu \mathrm{L}$ fresh medium per well in a 48-well culture plate. $1.5 \times 10^{5}$ cortical neurons in $75 \mu \mathrm{L}$ medium were seeded in each well. On DIV8, a scratch lesion (Tönges et al., 2011) was performed in a crossing pattern with a $200 \mu \mathrm{L}$ pipette tip in the 48-well plate. Afterwards, the plate was placed back into the incubator. At $6 \mathrm{~h}$ after scratch, cell lysates were prepared.

\subsubsection{Treatment with calpeptin and tianeptine}

To confirm the relation between CRMP2 and calpain in vitro, $0.1 \%$ DMSO or $50 \mu \mathrm{M}$ calpeptin in $0.1 \%$ DMSO was added to the culture medium at $1 \mathrm{~h}$ before scratch on DIV8. To investigate whether tianeptine could increase the levels of CRMP2, treatment with tianeptine alone or co-treatment with both tianeptine and calpeptin was applied to the cortical neurons. After treatment with $100 \mu \mathrm{M}, 500 \mu \mathrm{M}$ or $1000 \mu \mathrm{M}$ tianeptine, the cells were cultured in the incubator for $24 \mathrm{~h}$. For co-treatment, $50 \mu \mathrm{M}$ calpeptin in $0.1 \%$ DMSO was applied at 30 min before adding tianeptine. 


\subsubsection{HEK293 cells}

\subsubsection{HEK293 cells culturing}

HEK293 cells were maintained in the incubator at $37{ }^{\circ} \mathrm{C}$ with $5 \% \mathrm{CO}_{2}$ and $95 \%$ humidity. The culture medium was DMEM medium supplemented with FCS and penicillin/streptomycin. The medium was renewed every 2 days, and the cells were passaged when they reached confluence. To change medium, the cells were washed carefully with warm PBS, and then fresh medium was added. To passage cells, $1 \mathrm{~mL}$ trypsin was added directly on the cells in each flask after washing them with warm PBS. The flask was placed back into the incubator until the cells were detached from the flask. Fresh medium was added to stop trypsinization. The suspended cells were collected into a conical tube, and then centrifuged at $1000 \mathrm{rpm}$ for $1 \mathrm{~min}$. After aspirating the supernatant, the pellet was suspended in $20 \mathrm{~mL}$ fresh medium. The cell clumps were dispersed by pipetting up and down. $4 \mathrm{~mL}$ suspended cells were seeded into a new flask with $16 \mathrm{~mL}$ medium. For transfection, $500 \mu \mathrm{L}$ suspended cells and $2 \mathrm{~mL}$ medium were added to each well of a 6-well plate coated with PDL. The flask and plate were placed into the incubator for culturing.

\subsubsection{Calcium phosphate transfection}

After HEK293 cells in 6-well plate reached $70-80 \%$ confluence, calcium phosphate transfection was performed. $6 \mu \mathrm{gg}$ p.AAV-hSyn-CRMP2-hSyn-mcherry or p.AAV-hSyn-mcherry-hSyn were diluted to $108 \mu \mathrm{L}$ with $\mathrm{H}_{2} \mathrm{O}$ in $15 \mathrm{~mL}$ conical tube. For each sample, $12 \mu \mathrm{L} 2.5 \mathrm{M} \mathrm{CaCl}_{2}$ were added dropwise to the tube on the vortex. The time interval between drops was $3 \mathrm{~s}$. After adding the last drop of $\mathrm{CaCl}_{2}$, the tube was swirled on the vortex for 30 s. $120 \mu \mathrm{L} 2 \times$ BBS were added to the tube by the same way as adding $\mathrm{CaCl}_{2}$. After leaving the tube at RT for $15 \mathrm{~min}$, the medium in the 6-well plate was exchanged against $2 \mathrm{~mL}$ DMEM medium per well. After shortly swirling the mix with $\mathrm{HBS}, \mathrm{CaCl}_{2}$ and plasmid, the mix was added to the cells. $2 \mathrm{~h}$ after transfection, the cells were washed with DMEM medium. Afterwards, the cells were cultured with complete growing medium in the incubator. $24 \mathrm{~h}$ after transfection, a 50\% transfection rate was achieved. 


\subsubsection{Live imaging}

\subsubsection{Live imaging of acute axonal degeneration in vivo}

In vivo live imaging of $A A D$ in the optic nerve was performed as reported before (Koch et al., 2011). After surgical exposure of the optic nerve, the operating field was washed with warm Ringer's solution. The rat in the positioning setup was transferred and fixed on the stage of a Zeiss Examiner microscope. The 40x water immersion objective was positioned above the optic nerve. The cavity above the optic nerve was filled with Ringer's solution until the tip of the objective was surrounded by the solution. After verifying the integrity of the labeled axons, a crush lesion was performed. One to four areas $400 \mu \mathrm{m}$ proximal and distal to the crush site were selected for imaging. Z-stack images were taken of the chosen areas at $5,30,60,120,180,240,300,360 \mathrm{~min}$ after crush. To avoid bleaching, the fluorescence excitation was kept as weak and short as possible. During the whole period of imaging, the anesthesia of the rat was maintained by repeating anesthetic injections every 60 min based on close monitoring signs of awakening. To process the images, AxioVision 4.8 and CorelDraw X3 software were used. To quantify the time course of $A A D$, the axonal integrity ratio (AIR) was quantified for each axon. AIR represents a ratio of the sum lengths of the remaining axon divided by the total length of the initial axon.

\subsubsection{Live imaging of axonal degeneration in microfluidic chambers in vitro}

Live imaging of cortical neurons was performed in the microfluidic chamber system on DIV8. The cells were transfected with p.CMV-EGFP alone or co-transfected with p.CMV-EGFP and p.CMV-CRMP2. For imaging, the chambers were transferred to a microscope incubation system $\left(37^{\circ} \mathrm{C}, 5 \% \mathrm{CO}_{2}\right)$ attached to a Zeiss Axiovert inverted microscope. To monitor axonal changes after axotomy over time, 4 areas with EGFP fluorescent axons were chosen for imaging. Images were taken of the selected areas at 40x magnification. Imaging was performed before axotomy and up to $8 \mathrm{~h}$ after axotomy. For evaluation, the number of axonal bulbs $400 \mu \mathrm{m}$ proximal to the lesion site was quantified. Only the lesioned axons were included in these analyses. 


\subsubsection{Live imaging of mitochondrial movement in microfluidic chambers in vitro}

The mitochondrial transport was imaged in the microfluidic chamber system on DIV8. To check the kinetics of mitochondrial transport after axotomy, mitochondria were visualized by transduction with AAV.mito-RFP. To check the role of CRMP2 on mitochondrial transport after axotomy, $100 \mathrm{nmol}$ MitoTracker Green FM was added to each chamber on DIV7. The mitotracker was diluted in warm cortex medium. After removing the medium in the chamber, $200 \mu \mathrm{L}$ and $100 \mu \mathrm{L}$ diluted mitotracker were added to the soma and axonal sides of the chamber respectively. After placing the chamber in the incubator for $30 \mathrm{~min}$, the medium mixed with mitotracker was removed. The incubation time in the mitotracker was according to the datasheet of the mitotracker. $200 \mu \mathrm{L}$ and $100 \mu \mathrm{L}$ fresh medium were added to the soma and axonal sides of the chamber respectively. The volume difference between soma and axonal side resulted in a slow but continuous flow across the microgrooves. After washing overnight, the chamber was refilled with cortex medium.

On DIV8, mitochondria labeled with mitotracker or viral vector were imaged in the microscope incubation system $\left(37^{\circ} \mathrm{C}, 5 \% \mathrm{CO}_{2}\right)$ attached to a Zeiss inverted microscope. To monitor mitochondrial movement, a time-lapse movie was taken at $40 x$ magnification per area. Each movie was taken for $37 \mathrm{~s}$ with $500 \mathrm{~ms}$ exposure time. In each chamber, 4 areas were selected for imaging before axotomy and at different time points after axotomy. For evaluation, the mitochondrial movement in single axons $100 \mu \mathrm{m}$ proximal to the lesion site was quantified using the ImageJ plugin MultipleKymograph. Mitochondria with a speed of at least $0.07 \mu \mathrm{m} / \mathrm{s}$ were defined as motile mitochondria. Both percentage and speed of motile mitochondria were quantified. Only the lesioned axons were included for evaluation.

\subsubsection{Western blot analysis}

\subsubsection{Preparation of lysates}

$1 \mathrm{~mm}$ optic nerve segments proximal or distal to the crush site were dissected at the given time points before and after crush. Retinas were excised 4 weeks after intravitreal injections of viral vectors. Both retinas and optic nerves were dissected in PBS on ice immediately after the rats were sacrificed by $\mathrm{CO}_{2}$ insufflation. For Western blots of spectrin and CRMP2 in 
cortical neurons, cell lysates were collected at $6 \mathrm{~h}$ after scratch. To confirm CRMP2 overexpression mediated by p.CMV-CRMP2-flag, lysates of cortical neurons were collected 3 days after nucleofection. To check the effect of tianeptine on the levels of CRMP2, lysates of cortical neurons were prepared $24 \mathrm{~h}$ after treatment with tianeptine. To confirm CRMP2 overexpression mediated by p.AAV-hSyn-CRMP2-hSyn-mcherry, HEK293 cell lysates were prepared $24 \mathrm{~h}$ after calcium phosphate transfection.

To prepare lysates, tissues were immediately frozen in liquid nitrogen after dissection and then homogenized in ice-cold lysis buffer. For whole cell lysates, cells were washed with $5 \%$ glucose followed by ice-cold lysis buffer. Lysates were collected after scraping the cells off each well. The cell lysates were frozen and thawed for 2 times. Protein lysates of both tissues and cells were sonicated for $20 \mathrm{~s}$ at $4{ }^{\circ} \mathrm{C}$. After $10 \mathrm{~s}$, sonification was repeated for another time. After centrifugation at $1.32 \times 10^{4} \mathrm{rpm}$ for $30 \mathrm{~min}$ at $4{ }^{\circ} \mathrm{C}$, the supernatant was carefully transferred to a fresh tube.

\subsubsection{Western blot}

To prepare a SDS-PAGE gel, $10 \%$ separating gel solution was first added in the rack. Isopropanol was added to remove the bubbles. After about $40 \mathrm{~min}$, the separating gel was solidified. The $4 \%$ stacking gel solution was added on top of the separating gel and a comb was inserted. The stacking gel was solidified after $40 \mathrm{~min}$, and kept at $4{ }^{\circ} \mathrm{C}$ before use. To prepare each sample, equal amount (10-20 $\mu \mathrm{g})$ of proteins were diluted in $16 \mu \mathrm{L}$ lysis buffer. Each diluted sample was mixed with $4 \mu \mathrm{L}$ Laemmli buffer $(5 \mathrm{x})$, and then boiled at $95^{\circ} \mathrm{C}$ for 5 min. For gel electrophoresis, the gel was placed in the chamber with electrophoresis buffer and the comb was removed carefully. A protein ladder $(5 \mu \mathrm{L})$ was loaded in the first lane and the samples were loaded in the other lanes. The remaining empty lanes were loaded with a mixture of loading buffer with lysis buffer. The gel was first run at 30-50 V for about 40 min until the dye front entered the separating gel and then at $120 \mathrm{~V}$ until the dye front nearly ran off the bottom of the gel. For transferring membranes, a polyvinylidene difluoride (PVDF) or nitrocellulose (NC) membrane was cut in the size of $6 \mathrm{~cm} \times 9 \mathrm{~cm}$. PVDF membrane needed to be activated in methanol for $30 \mathrm{~s}$ before use. A transfer "sandwich" was assembled with 
sponge, filter paper, membrane and gel after wetting them in the transfer buffer. By rotating a tube on top of the "sandwich", air bubbles were removed. By performing the transfer at $100 \mathrm{~V}$ at $4{ }^{\circ} \mathrm{C}$ for 1 to $2 \mathrm{~h}$, proteins were blotted to the membrane. The blotted membrane was blocked with $5 \%$ BSA or $5 \%$ milk in TBST for $1 \mathrm{~h}$ at RT. After washing in TBST for $3 \times 10$ min, the membrane was incubated with primary antibodies: anti-spectrin (1:500), anti-CRMP2 (1:1000), anti-Atg5 (1:300), anti-Beclin-1 (1:1000), anti-Map-2 (1:1000), anti-Tau (1:1000), anti-Dynein IC (1:500), anti-Flag M2 antibody (1:1000), and anti-GAPDH (1:2500) at $4{ }^{\circ} \mathrm{C}$ overnight. The membrane was then washed with TBST for $3 \times 10 \mathrm{~min}$, and was incubated with HRP-coupled secondary antibodies at RT for $1 \mathrm{~h}$. After rinsing in TBST for $3 \mathrm{x}$ 10 min, enhanced chemiluminescence $(E C L)$ mixture was prepared by mixing $E C L$ reagent 1 and 2 in a ratio of 1:1. The membrane was incubated with the ECL-mix for $1 \mathrm{~min}$ and transferred to the dark room. In the dark room, a film was placed on top of the membrane in the cassette. After applying different exposure times from seconds to minutes, the film was developed with a Curix 60 Developer. The band intensity of target proteins was quantified by ImageJ 1.49a software (NIH).

\subsubsection{Immunofluorescence analysis}

\subsubsection{Immunohistochemistry}

Optic nerves were dissected on ice before or $1 \mathrm{~h}$ after ONC. The dissected optic nerves were fixed in $4 \%$ PFA at $4{ }^{\circ} \mathrm{C}$ overnight, and then transferred to $30 \%$ sucrose in PBS at $4{ }^{\circ} \mathrm{C}$ for $48 \mathrm{~h}$. The tissues were frozen at $-20^{\circ} \mathrm{C}$ until $16 \mu \mathrm{m}$ thick longitudinal sections were cut using a cryostat. The sections were mounted onto glass slides. After removing the air bubbles, slides with sections were dried at $37^{\circ} \mathrm{C}$ for $20 \mathrm{~min}$ and stored at $-20^{\circ} \mathrm{C}$ until further use.

For staining, $0.05 \mathrm{M}$ Tris/1.5\% $\mathrm{NaCl}(\mathrm{pH} 7.6)$ was used as washing buffer. All the washing steps were performed with gently shaking at RT. Slides were first thawed at $37^{\circ} \mathrm{C}$ for $20 \mathrm{~min}$, and then rehydrated in washing buffer at RT for $45 \mathrm{~min}$. For antigen retrieval, the sections were incubated in $0.05 \mathrm{M} \mathrm{Tris} / 1.5 \% \mathrm{NaCl}(\mathrm{pH} 9)$ in the water bath at $60{ }^{\circ} \mathrm{C}$ for $4 \mathrm{~h}$. After washing for $5 \mathrm{~min}$, the slides were permeabilized in methanol at $-20^{\circ} \mathrm{C}$ for $10 \mathrm{~min}$. The slides 
were then washed for $3 \times 10$ min. After incubation in $10 \%$ Triton X-100 for 15 min at RT, the

slides were washed for another $5 \mathrm{~min}$. The non-specific staining was blocked with Dako antibody diluent at RT for $30 \mathrm{~min}$. Primary antibodies including anti-Smi31 (1:1000) and anti-cleaved spectrin (1:5000) were diluted in Dako antibody diluent and applied to the sections at $4{ }^{\circ} \mathrm{C}$ overnight. After washing for $5 \times 10 \mathrm{~min}$, sections were incubated with secondary antibodies at RT for $1 \mathrm{~h}$. DAPI was applied at RT for $10 \mathrm{~min}$ after another 2 times of washing. After washing for 2 more times, the sections were embedded with Mowiol. To have an overview of the stained optic nerve, 20x magnification images were taken with a Zeiss AxioPlan microscope. The images were put together with Coreldraw software. For evaluation, the line plot profile tool of ImageJ 1.49a was used to measure the intensity of cleaved spectrin along the optic nerves. To evaluate the intensity of axonal cleaved spectrin, images were taken with the pseudo-confocal Zeiss ApoTome device at $63 x$ magnification. 8 images were taken in the area proximal or distal $300 \mu \mathrm{m}$ to the crush in each nerve. 3 optic nerves were included per group and 5 sections per optic nerve were evaluated in the analysis.

\subsubsection{Immunocytochemistry}

Immunocytochemistry of cortical neurons in microfluidic chambers after co-transfection with p.CMV-EGFP and p.CMV-CRMP2-flag was performed on DIV8. The cells were washed for 5 min in PBS at RT and then fixed in 4\% PFA at RT for 10 min. After washing for 3 times, the cells were permeabilized in $0.25 \%$ triton-100 at RT for 10 min and then blocked with $5 \%$ goat serum at RT for 30 min. Primary antibody against Flag-M2 (1:500) was applied at $4{ }^{\circ} \mathrm{C}$ overnight. After washing for 3 times, the cells were incubated with goat anti-mouse cy3 secondary antibody at RT for $1 \mathrm{~h}$. The cells were washed for 3 more times and incubated with DAPI at RT for 10 min. After another washing in PBS and deionized $\mathrm{H}_{2} \mathrm{O}$, the chamber was detached from the coverslip, and the cells on the coverslip were mounted with Mowiol. Images were taken with a Zeiss AxioPlan microscope at 40x magnification. 


\subsubsection{RNA isolation and analysis}

\subsubsection{RNA isolation}

To confirm CRMP2 overexpression by the plasmid p.AAV-hSyn-CRMP2-hSyn, total RNA was isolated on DIV8 after transfection of cortical neurons. To confirm CRMP2 overexpression by the viral vector AAV-hSyn-CRMP2-hSyn in vitro, total RNA was isolated on DIV8 after transduction of cortical neurons. To confirm CRMP2 overexpression by AAV-hSyn-CRMP2-hSyn in vivo, total RNA of optic nerves was isolated 4 weeks after intravitreal injection of the viral vector.

For homogenization of cortical neurons, cells were washed with ice cold PBS once. After removing the PBS, Trizol reagent was added to each well. After $5 \mathrm{~min}$, the cells were pipetted up and down for 3 times. The cells from 5 wells per sample were collected to $2 \mathrm{~mL}$ tube. For homogenization of tissues, the dissected optic nerve was immediately frozen in liquid nitrogen. As soon as it was taken out of liquid nitrogen, each sample was homogenized in $500 \mu \mathrm{L}$ Trizol completely. After $3 \mathrm{~min}$, another $500 \mu \mathrm{L}$ Trizol was added to each sample. Further homogenization was performed by pipetting up and down for 10 times.

After homogenization of both optic nerves and cortical neurons, $100 \mu \mathrm{L}$ 1-Bromo-3-chlor-propane were added per $1 \mathrm{~mL}$ Trizol. The sample was inverted at RT for 15 times. After $3 \mathrm{~min}$, the sample was centrifuged at $12000 \mathrm{rcf}$ at $4{ }^{\circ} \mathrm{C}$ for $15 \mathrm{~min}$. The upper aqueous layer was collected to another tube. $500 \mu \mathrm{L}$ isopropanol and $1 \mu \mathrm{L}$ glycolblue were added to the aqueous layer and mixed by inverting the tube for 15 times. The sample was kept at $-20^{\circ} \mathrm{C}$ overnight followed by centrifugation at $12000 \mathrm{rcf}$ at $4{ }^{\circ} \mathrm{C}$ for $20 \mathrm{~min}$. After discarding the supernatant, the RNA pellet was washed with ice cold $75 \%$ ethanol. DEPC $\mathrm{H}_{2} \mathrm{O}$ was added to the air-dried pellet and heated at $55^{\circ} \mathrm{C}$ for 2 min. Finally, the concentration of dissolved RNA was measured using a Nanodrop spectrophotometer.

\subsubsection{Reverse transcription}

QuantiTect reverse transcription kit was used to perform reverse transcription according to its datasheet. 2 samples were prepared per condition. In each sample, $2 \mu \mathrm{L}$ gDNA wipeout buffer and $250 \mathrm{ng}$ template RNA were prepared. DEPC $\mathrm{H}_{2} \mathrm{O}$ was added to reach the total 
volume $14 \mu \mathrm{L}$. After incubation at $42{ }^{\circ} \mathrm{C}$ for $2 \mathrm{~min}$, they were kept on ice. After genomic DNA elimination, each sample was mixed with $1 \mu \mathrm{L}$ Transcriptase, $4 \mu \mathrm{L}$ Quantiscript RT buffer and $1 \mu \mathrm{L} \mathrm{RT}$ primer mix. The samples were then incubated at $42{ }^{\circ} \mathrm{C}$ for $15 \mathrm{~min}$ and at $95{ }^{\circ} \mathrm{C}$ for 3 min. Finally, they were kept at $-20^{\circ} \mathrm{C}$ or on ice for further steps.

\subsubsection{Real-Time quantitative PCR}

The primer of GAPDH was Rn_Gapd_1_SG (NM_017008) from Qiagen. The human specific CRMP2 primers were designed by basic local alignment search tool (blast) and their sequences were: CRMP2 forward primer: 5'-CGTGAATCGTGCCATCACCA-3'; CRMP2 reverse primer: 5'-AGTAATGGGAGCCGTCCGTT-3'. QuantiTect SYBR green PCR kit was used to perform Real-Time quantitative PCR. Each reaction with CRMP2 primer was prepared with $10 \mu \mathrm{L}$ quantiTect SYBR Green PCR Master Mix, $1.25 \mu \mathrm{L}$ template DNA after reverse transcription, $0.5 \mu \mathrm{M}$ CRMP2 forward primer and $0.5 \mu \mathrm{M}$ CRMP2 reverse primer. For each reaction with GAPDH primer, $0.5 \mu \mathrm{M}$ GAPDH primer was added instead of CRMP2 primers. RNase free $\mathrm{H}_{2} \mathrm{O}$ was added to reach the final volume $20 \mu \mathrm{L}$ for each reaction. Each condition included 3 repeats. No template control was included for the reaction of CRMP2 or GAPDH primer. After pipetting template CDNA into a PCR plate, the mixture of quantiTect SYBR Green PCR Master Mix, primers and RNase free $\mathrm{H}_{2} \mathrm{O}$ was added. The PCR plate was placed in a thermal cycler, and the program was started. Bio-Rad CFX manager software was used to quantify the expression of CRMP2 mRNA relative to the mRNA expression of GAPDH according to the Bio-Rad CFX manager software. 3 independent cultures or optic nerves were included in each group.

\subsubsection{Proteomics analysis}

Proteomics analysis was performed in unlesioned optic nerves and lesioned optic nerves at $6 \mathrm{~h}$ after crush lesion. Experimental groups included unlesioned optic nerves, proximal and distal part of optic nerves at $6 \mathrm{~h}$ after crush. To prepare protein lysates, $1 \mathrm{~mm}$ optic nerve segments proximal or distal to the crush site were dissected on ice. To minimize 
intra-animal variability, 4 independent $1 \mathrm{~mm}$ optic nerve segments were pooled into one sample in each group. Further preparation procedures for protein lysates were performed as with Western blot analysis. After the protein concentration was measured by BCA assay, $45 \mu \mathrm{g}$ proteins were diluted in loading buffer to a final volume of $38 \mu \mathrm{L}$ in each sample. After heating samples at $95^{\circ} \mathrm{C}$ for $5 \mathrm{~min}$, they were transferred on ice to the proteomics service facility of the University Medicine Göttingen for further analysis.

After reconstituting the samples in 1x NuPAGE LDS Sample Buffer, they were separated on 4-12\% NuPAGE Novex Bis-Tris Minigels. For visualization purposes, the gels were stained with Coomassie Blue. Regardless of staining, each lane was sliced into 23 equidistant parts. After washing, gel slices were reduced with DTT, and alkylated with 2-iodoacetamide. After digesting the gel slices with trypsin overnight, the resulting peptide mixtures were extracted, dried in a SpeedVac, and reconstituted in $2 \%$ acetonitrile/0.1\% formic acid (v:v). Afterwards, they were prepared for nanoLC-MS/MS as described before (Atanassov and Urlaub, 2013).

For mass spectrometric analysis, samples were enriched on a self-packed reversed phase-C18 precolumn (0.15 mm ID x 20 mm, Reprosil-Pur120 C18-AQ, $5 \mu \mathrm{m}$ ). They were then separated on an analytical reversed phase-C18 column $(0.075 \mathrm{~mm}$ ID $\times 250 \mathrm{~mm}$, Reprosil-Pur 120 C18-AQ, $3 \mu \mathrm{m})$. A 30 min linear gradient of 5-35\% acetonitrile/0.1\% formic acid at $300 \mathrm{~nL} / \mathrm{min}$ ) was used during the enrichment and separation of the samples. By using a data-dependent acquisition method, the eluent was analyzed on a TripleTOF $5600+$ hybrid quadrupole/time-of-flight (QqTOF) mass spectrometer equipped with a nanospray III ion source and operated under Analyst TF1.6 software build 6211. Each experimental cycle was of the following form: To select up to the 15 most abundant peptide precursors of charge states 2 to 4 above a 250 cps intensity threshold, one full MS scan across the $350-1250 \mathrm{~m} / \mathrm{z}$ range was acquired at a resolution of 30,000 FWHM and an accumulation time of $250 \mathrm{~ms}$. Precursors were then isolated at 0.7 FWHM isolation width and fragmented with nitrogen at default rolling collision energy settings. The resulting product ion spectra recorded across the $180-1600 \mathrm{~m} / \mathrm{z}$ range at a resolution of $17,500 \mathrm{FWHM}$ and an accumulation time of $100 \mathrm{~ms}$. For the following $9 \mathrm{~s}$, selected precursor $\mathrm{m} / \mathrm{z}$ values were excluded. Two technical replicates were performed per sample. 
For data analysis, ProteinPilot 5.0 software rev4769 was used for protein identification. Proteins were identified against the UniProtKB rat reference proteome v2015.02 (58766 protein entries) and a set of 51 contaminants commonly identified in Christof Lenz's lab. The research was performed at "thorough" search settings, with trypsin as enzyme and iodoacetamide as cysteine blocking agent. For further processing, results were exported into mzldentML 1.1.0 format. To validate MS/MS based peptide and protein identifications, scaffold software version 4.4.1.1 was used. Peptide identifications were accepted if they could be established at greater than $95.0 \%$ probability by the Paragon algorithm (Shilov et al., 2007). Protein identifications were accepted if they could be established at greater than $31.0 \%$ probability to achieve an FDR less than $1.0 \%$ and contained at least 2 identified peptides. The Protein Prophet algorithm was used to assign protein probabilities (Nesvizhskii et al., 2003). Proteins that contained similar peptides and could not be differentiated based on MS/MS analysis alone were grouped to satisfy the principles of parsimony. Proteins sharing significant peptide evidence were grouped into clusters. Proteins annotation was performed with GO terms from NCBI downloaded Feb 23, 2015 (Ashburner et al., 2000). To allow for the calculation of low abundance protein ratios, a minimum value of 3 spectral counts was introduced where necessary to avoid division by zero issues. Relative quantification of proteins in the samples was achieved by Analysis of Variance (ANOVA) of normalized Spectral Counts. A Benjamini-Hochberg-corrected $p$ value of 0.05 was used to judge significance. For the significantly regulated proteins, a STRING ( 10 for rat) database search was performed by Caroline C. Friedel (Institute for Informatics, Ludwig-Maximilians-University Munich) to identify the proteins linked with CRMP2. Fold changes of the protein levels in proximal or distal part at $6 \mathrm{~h}$ after ONC were calculated relative to control group. The protein levels were considered changed when they had a fold change $\geq 1.3$ or $\leq-1.3$ at $6 \mathrm{~h}$ after ONC.

\subsubsection{Statistical analysis}

Statistical analysis was performed using SPSS software. For two-group experiments, the statistical difference was compared using independent samples t-test. For the experiments with more than two groups, statistical comparison was performed using one-way ANOVA 
followed by Dunnett's post-hoc test. All data are presented as mean \pm standard error of the mean (SEM). Differences are considered different as indicated with $* P<0.05, * * P<0.01$, ${ }^{* * *} P<0.001$. The performed statistical test and repeats of each experiment are shown in the figure legends. 


\section{Results}

\subsection{The role of calpain in acute axonal degeneration of the optic nerve}

\subsubsection{Time course and localization of calpain activation during acute axonal degeneration} in the optic nerve

To investigate the involvement of calpain during $A A D$, we analyzed the expression levels of the $145 \mathrm{kDa}$ breakdown product (BDP) of spectrin, which is specifically derived from calpain cleavage (Wang, 2000). Western blot of spectrin in the optic nerve was performed before crush and at different time points after crush. For protein lysates preparation, two regions $1 \mathrm{~mm}$ proximal and distal to the crush site were dissected from the lesioned optic nerves. As control, the corresponding areas of native optic nerves were dissected. Expression levels of the $145 \mathrm{kDa}$ spectrin-BDP as a marker for calpain activation showed a significant time-dependent increase on both proximal and distal sides of the crush (Figure 3.1A-B).

A
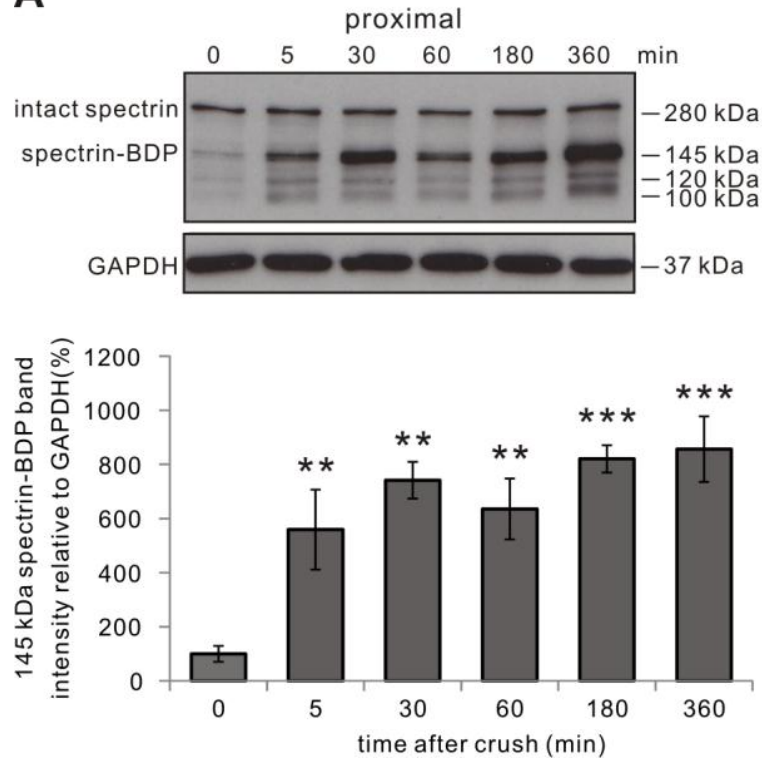

B
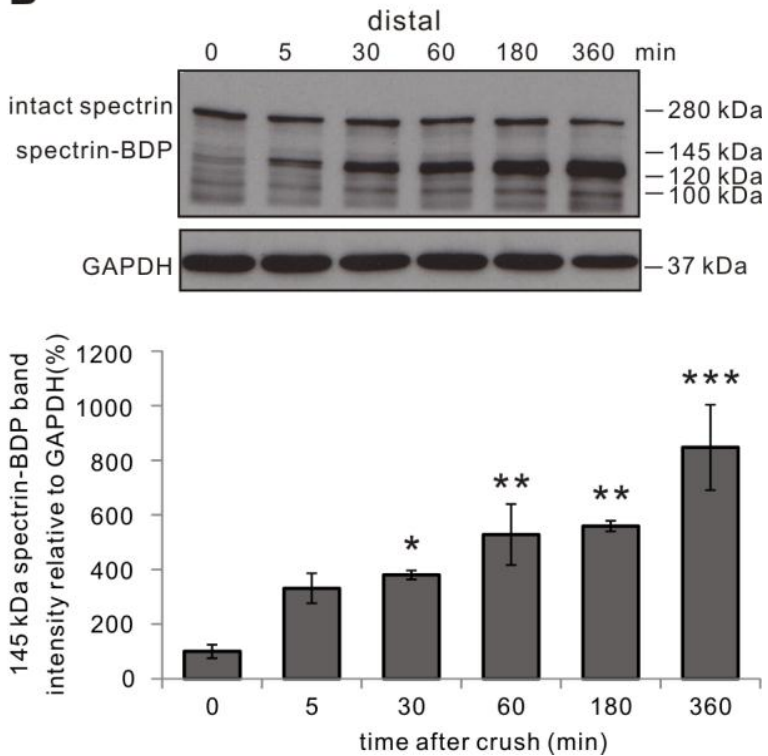

Figure 3.1 Immunoblot analysis of calpain-mediated spectrin proteolysis during acute axonal degeneration after optic nerve crush in vivo.

(A, B) Representative immunoblots of spectrin proximal (A) and distal (B) to the crush site in the upper 
panels. The protein lysates were prepared from native optic nerves ( 0 min after crush) and in the optic nerves at indicated time points after crush. BDP = breakdown product. The $145 \mathrm{kDa}$ spectrin-BDP is specifically produced by calpain cleavage. Below, the graphs show the quantification of $145 \mathrm{kDa}$ spectrin-BDP band intensity proximal (A) and distal (B) to the crush site. GAPDH was used as the loading control. 3-6 optic nerves are included in each time point. Error bars represent the standard error of the mean (SEM). Statistical significance is compared to 0 min after crush: ${ }^{*} p<0.05,{ }^{* *} p<0.01,{ }^{* * *} p<0.001$ by one-way ANOVA and Dunnett's test.

In order to further confirm the results and to specify the location of calpain activation, we performed an immunohistochemical staining of optic nerve sections with an antibody that specifically detects the calpain-generated spectrin-BDP (Roberts-Lewis et al., 1994). A Smi31-antibody (staining phosphorylated axonal neurofilaments) was used to label the axons. Longitudinal sections were prepared from optic nerves at $1 \mathrm{~h}$ after crush and native optic nerves (control group). The staining intensity along the longitudinal optic nerve sections was quantified by the plot profile tool of ImageJ. Compared to control group, we observed a higher staining intensity of the spectrin-BDP already at $1 \mathrm{~h}$ after ONC in the regions close to the crush site (Figure 3.2A). Interestingly, it was confined to the area $300 \mu \mathrm{m}$ proximal and distal to the crush site, which is the corresponding region affected by AAD (Knöferle et al., 2010). Next, we quantified the spectrin-BDP staining intensity specifically in the Smi31-labeled axons in this area. We found that the intra-axonal spectrin-BDP staining intensity was significantly increased in both proximal and distal parts compared to the unlesioned control (Figure 3.2B).

In summary, these results demonstrate that calpain is activated early during AAD after ONC and that the activation localizes specifically intraaxonally within the area that is affected by $A A D$. 
A
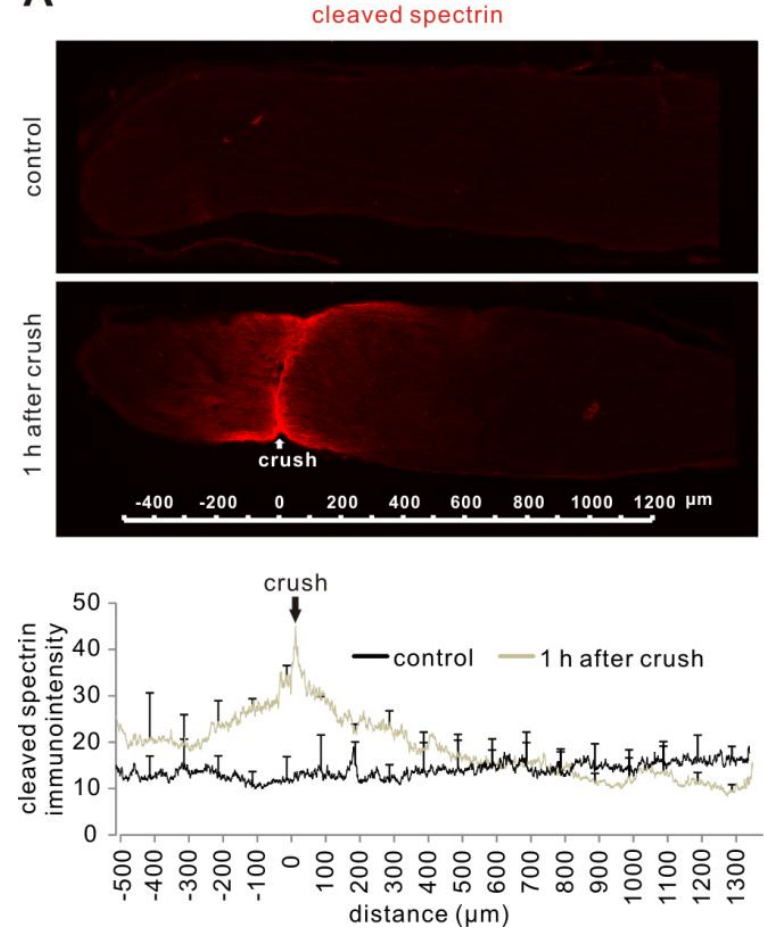

B

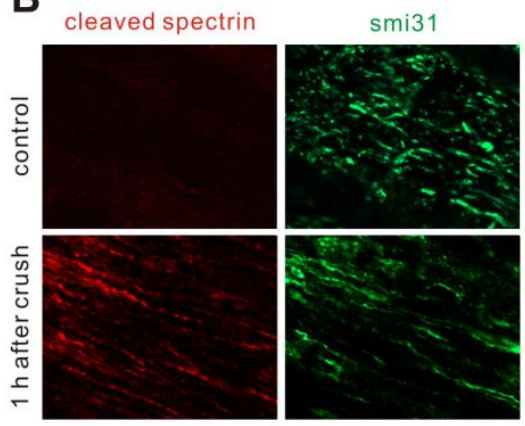

merged
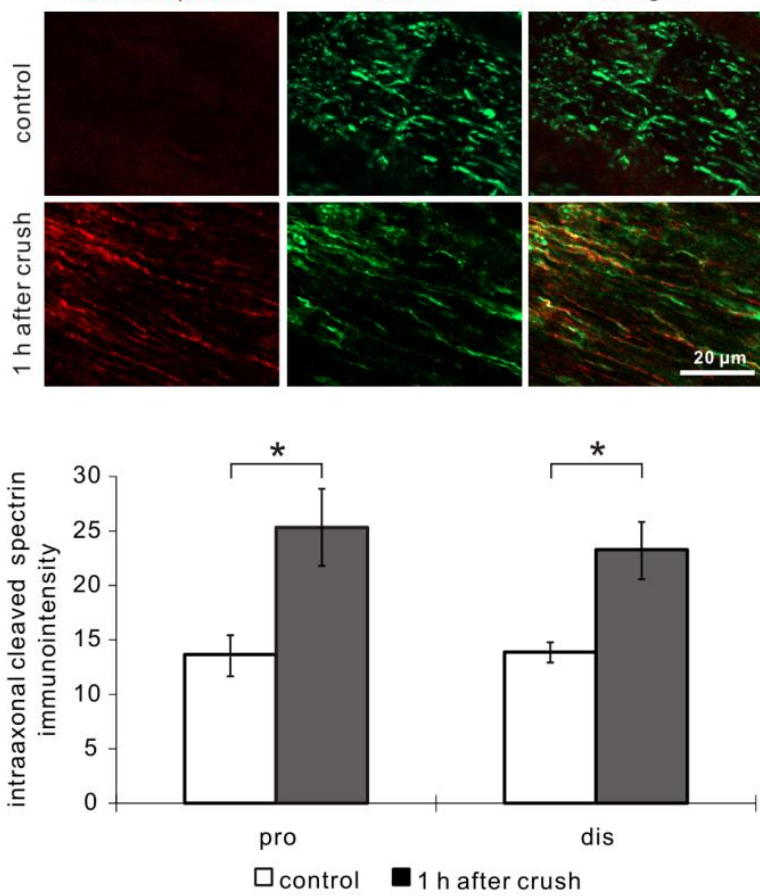

Figure 3.2 Immunohistochemical analysis of calpain-mediated spectrin proteolysis during acute axonal degeneration after optic nerve crush in vivo.

(A) Representative images (20x) immunostained with an antibody specifically recognizing cleaved spectrin in a native optic nerve (control) and an optic nerve at $1 \mathrm{~h}$ after crush. The antibody against cleaved spectrin selectively detects the spectrin-BDP generated by active calpain. Below, the graph shows the quantification of the staining intensity along the longitudinal sections by the plot profile tool of ImageJ.

(B) Higher magnification images (63x) of the representative areas in a native optic nerve and in an optic nerve at $1 \mathrm{~h}$ after crush. The images were immunostained against cleaved spectrin and the axonal marker Smi31 in the area $300 \mu \mathrm{m}$ proximal to the crush site. At the bottom, the graph shows the quantification of the spectrin-BDP staining intensity in the axons proximal and distal $300 \mu \mathrm{m}$ to the crush site. 3 optic nerves are included in each group. ${ }^{*} p<0.05$ by independent samples t-test.

\subsubsection{Live imaging of crush-induced acute axonal degeneration after calpain inhibition in} vivo

Since calpain was activated during crush-induced AAD, we determined whether calpain 
inhibition would interfere with the time course of AAD. For calpain inhibition, the pharmacological calpain inhibitor calpeptin was employed (Tsujinaka et al., 1988). The intravitreal injection of $10 \mathrm{mM}$ calpeptin in 7\% DMSO at $2.5 \mathrm{~h}$ before crush was found to effectively inhibit calpain activation induced by crush lesion in the optic nerve. This was confirmed by Western blot analysis of the calpain-generated $145 \mathrm{kDa}$ spectrin-BDP. The protein lysates were prepared from two regions of optic nerves $1 \mathrm{~mm}$ proximal and distal to the crush site at $6 \mathrm{~h}$ after crush. The analysis showed that $10 \mathrm{mM}$ calpeptin in $7 \%$ DMSO significantly attenuated the increase of the $145 \mathrm{kDa}$ spectrin-BDP at $6 \mathrm{~h}$ after ONC compared to $7 \%$ DMSO treatment (control) (Figure 3.3A-C).

To assess the time course of $A A D$, we performed an in vivo live imaging of the rat optic nerve, which was previously developed by our group (Koch et al., 2011). To visualize the axons in the optic nerve, intravitreal injection of AAV.hSyn-EGFP was performed 2 weeks before live imaging (Figure 4A). For calpain inhibition, intravitreal injection of $10 \mathrm{mM}$ calpeptin in 7\% DMSO or 7\% DMSO (control) was performed at $2.5 \mathrm{~h}$ after ONC. Single axons $500 \mu \mathrm{m}$ proximal and distal to the crush site were imaged before ONC and at various time points after ONC. For each labeled axon, the axonal integrity ratio (AIR) was quantified, which is defined as the sum lengths of the remaining axonal fragments at a given time-point divided by the total initial axon length. A higher AIR represents a more intact axon (Knöferle et al., 2010; Koch et al., 2011). In both proximal and distal parts, calpeptin treatment significantly attenuated the time course of AAD compared to control group (Figure 3.4A-E). This attenuation of AAD by calpeptin treatment started to be significant at 120 min after ONC, and continued up to $360 \mathrm{~min}$ after ONC. The axon stabilizing effect of calpeptin was more pronounced in the proximal part (AIR at $360 \mathrm{~min}$ : control: $0.23 \pm 0.06$; calpeptin: $0.87 \pm$ 0.07 ) as compared to the distal part (AIR at $360 \mathrm{~min}$ : control: $0.24 \pm 0.03$; calpeptin: $0.41 \pm$ 0.06) (Figure 3.4A-E). In summary, these results demonstrate that calpain inhibition attenuates $A A D$ after ONC in vivo, especially in the proximal part. 


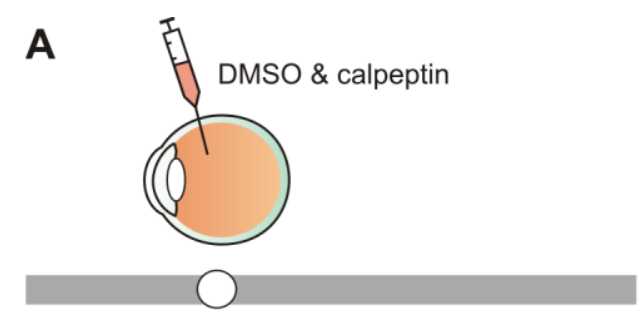

$-2.5 \mathrm{~h}$

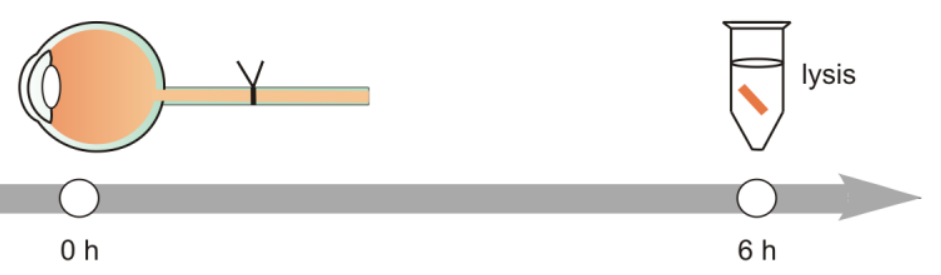

B
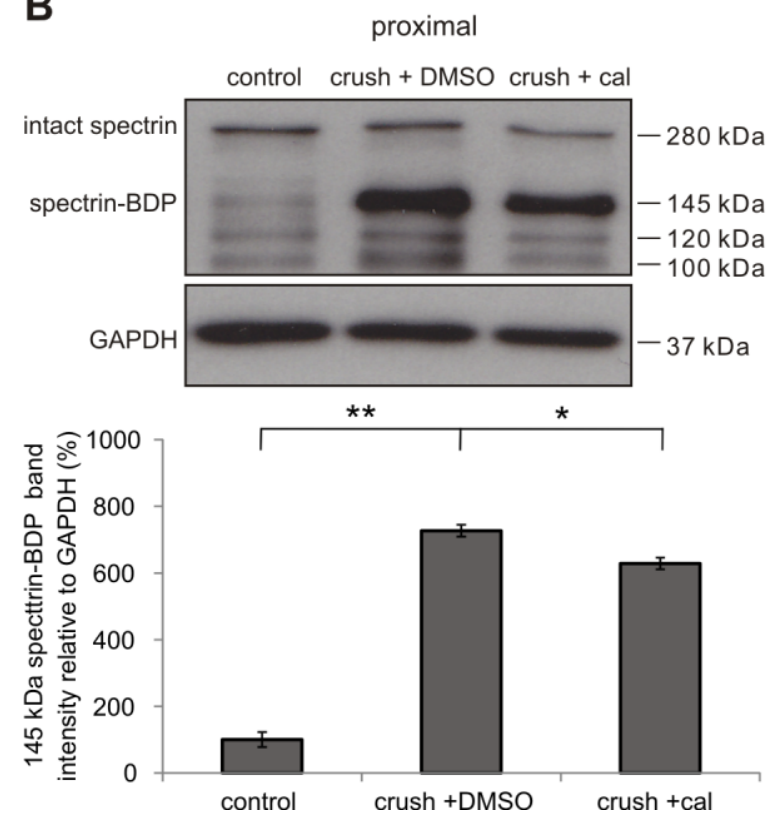

C
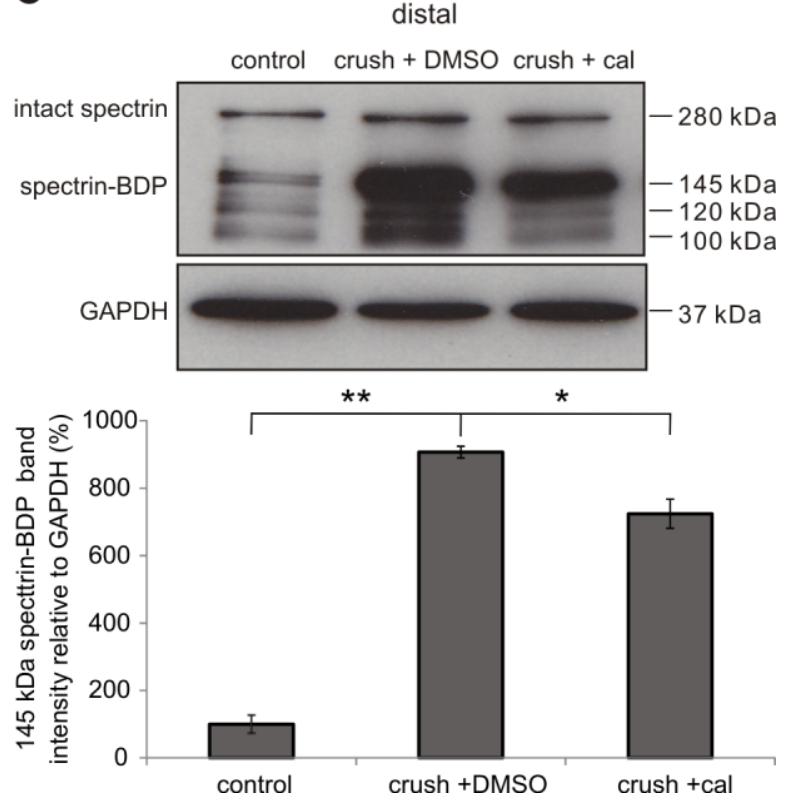

Figure 3.3 Effect of the calpain inhibitor calpeptin on calpain-mediated spectrin proteolysis after optic nerve crush in vivo.

(A) The experimental setup and time scale: Intravitreal injection of $10 \mathrm{mM}$ calpeptin in $7 \%$ DMSO or $7 \%$ DMSO ( $3 \mu \mathrm{L}$ for each eye) was performed $2.5 \mathrm{~h}$ before ONC. Segments of optic nerves were dissected from two areas $1 \mathrm{~mm}$ proximal and distal parts to the crush site, and protein lysates were prepared at $6 \mathrm{~h}$ after ONC.

(B, C) Representative immunoblots of spectrin proximal (B) and distal (C) to the crush site in the upper panels. $\mathrm{Cal}=$ calpeptin. Experimental groups include native optic nerves (control), optic nerves at $6 \mathrm{~h}$ after ONC pretreated with 7\% DMSO (crush + DMSO), and optic nerves at $6 \mathrm{~h}$ after ONC pretreated with $10 \mathrm{mM}$ calpeptin in 7\% DMSO (crush + cal). At the bottom, the $145 \mathrm{kDa}$ spectrin-BDP band intensity was quantified and normalized to GAPDH in both proximal (B) and distal (C) parts. 3 independent optic nerves are included in each group. Error bars represent the standard error of the mean (SEM). ${ }^{*} p<0.05,{ }^{* *} p<$ 
0.01 by one-way ANOVA and Dunnett's test.

A
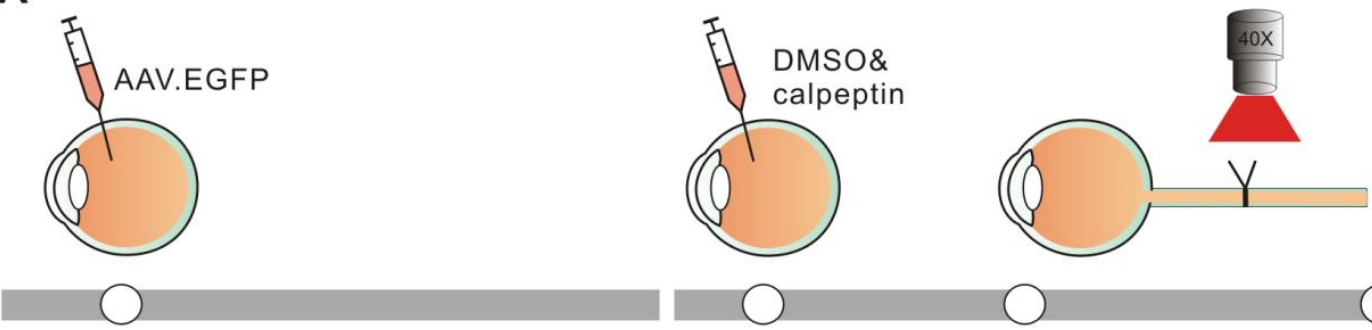

$-14 d$

$-2.5 \mathrm{~h}$

$\mathrm{oh}$

$6 \mathrm{~h}$

B
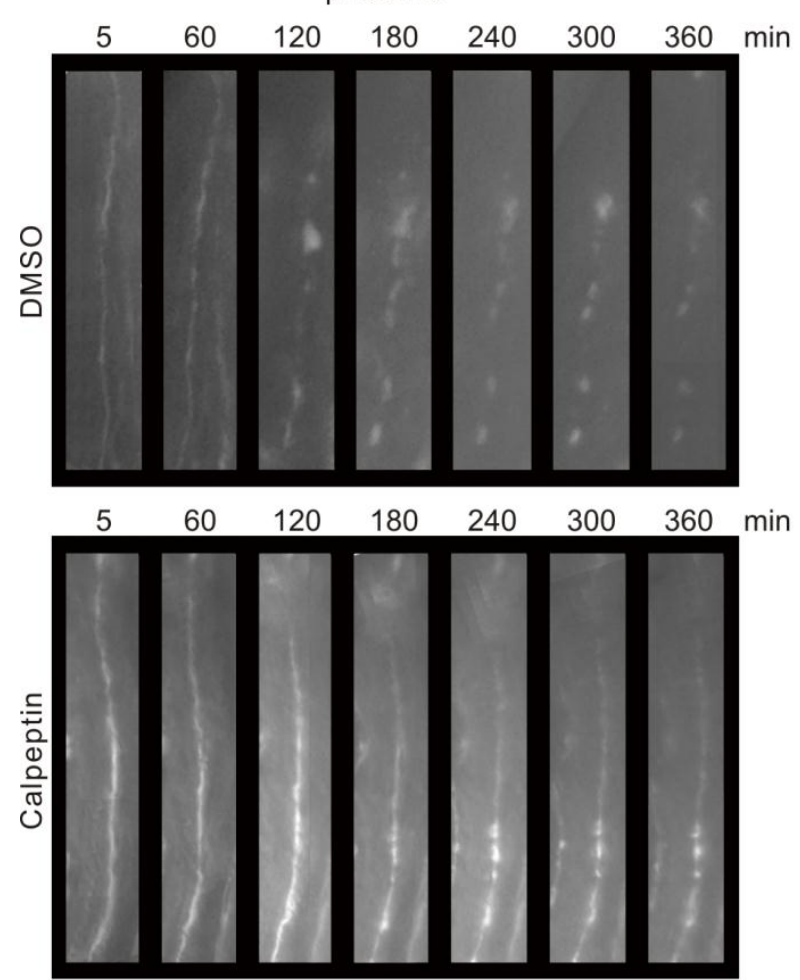

$50 \mu \mathrm{m}$

D

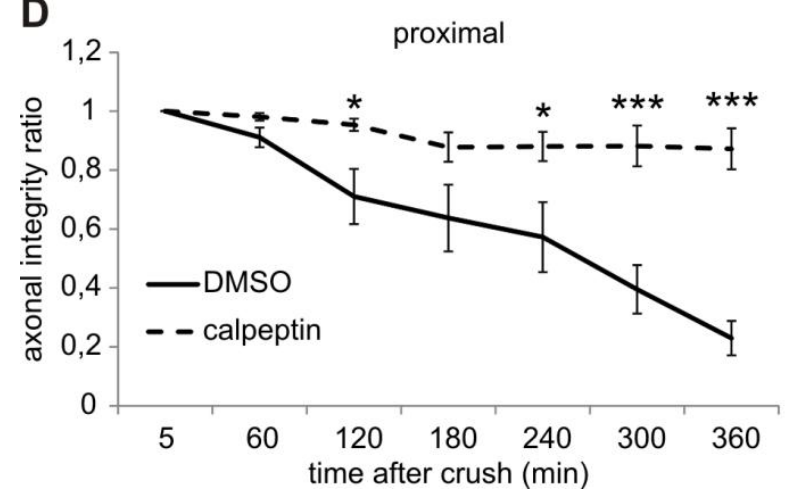

C
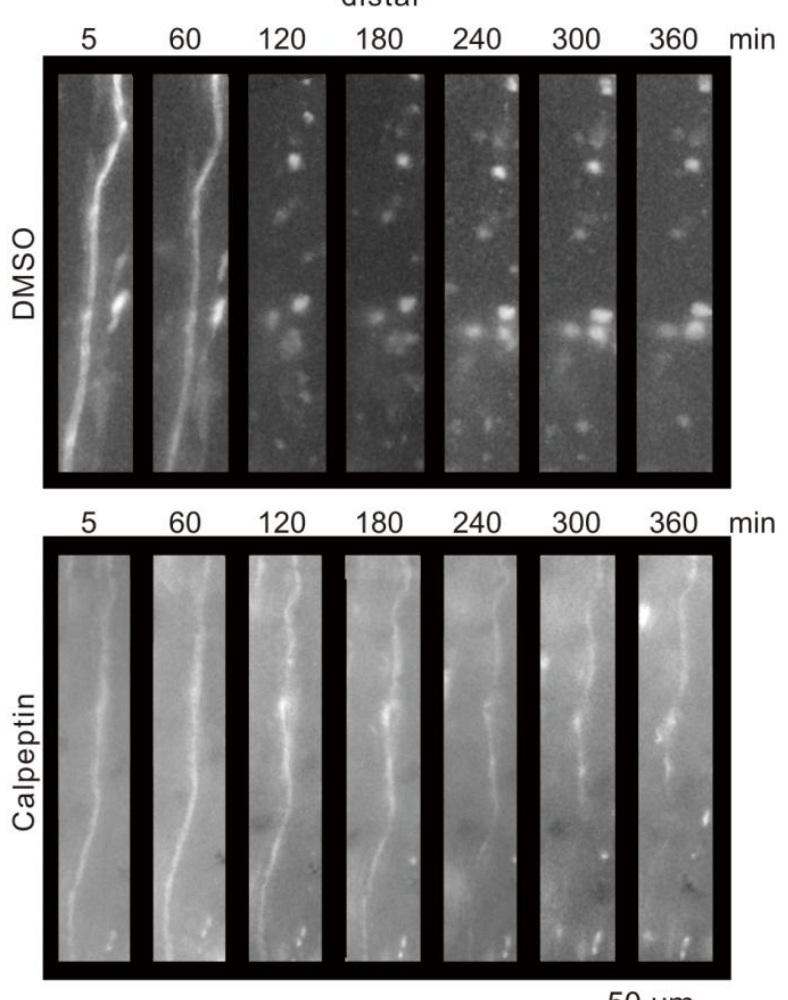

$50 \mu \mathrm{m}$

E

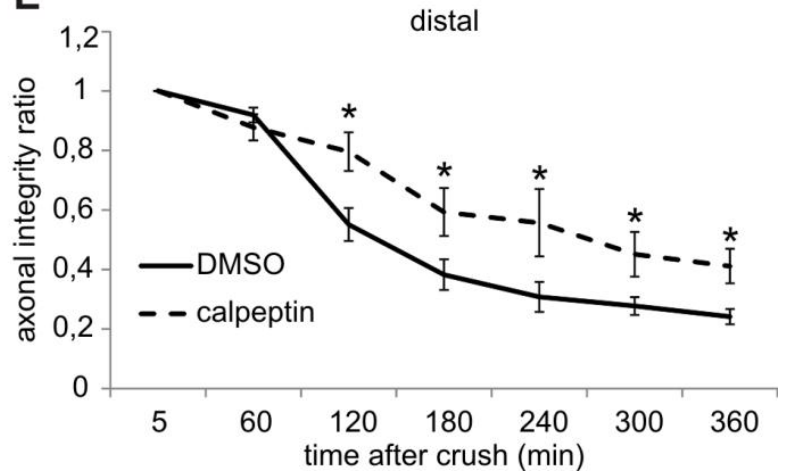

Figure 3.4 Time course of $A A D$ in the optic nerve after calpain inhibition in vivo.

(A) Experimental setup and time scale: Intravitreal injection of AAV.EGFP was performed to visualize 
retinal ganglion cell axons. After two weeks, intravitreal injection of $3 \mu \mathrm{l}$ 7\% DMSO or $10 \mathrm{mM}$ calpeptin in 7\% DMSO was performed $2.5 \mathrm{~h}$ before ONC. Single axons in the optic nerve were imaged in the area spanning $400 \mu \mathrm{m}$ to both sides from the crush. Z-stack images were taken before ONC and over $6 \mathrm{~h}$ after ONC.

(B, C) Representative images of axonal changes proximal (B) and distal (C) to the lesion site at the given time points after ONC. The upper panels represent control group (7\% DMSO) and the bottom panels show the calpeptin treatment group.

(D, E) Time course of axonal integrity ratios proximal (D) and distal (E) to the lesion at the indicated time points after ONC. The axonal integrity ratio is the sum length of the remaining axonal fragments at a given time-point divided by the initial total axon length. Statistical significance is compared between calpeptin treatment group and control group at each corresponding time point. 5-6 rats are included in each group. Error bars represent the standard error of the mean (SEM). ${ }^{*} p<0.05,{ }^{* * *} p<0.001$ by independent samples t-test.

\subsubsection{Characterization of downstream targets of calpain during acute axonal degeneration}

To identify biologically relevant downstream targets of activated calpain during AAD, we evaluated the expression levels of several proteins in the rat optic nerve at $6 \mathrm{~h}$ after lesion compared to the unlesioned control. We chose proteins that had been described as calpain cleavage targets in other model systems before (Atalay et al., 2007; Liu et al., 2011; Russo et al., 2011; Yoon et al., 2008; Yousefi et al., 2006; Zhang et al., 2007). Among these, we selected the proteins that are known to be involved in central molecular pathways of axonal degeneration like autophagy (ATG5, beclin-1), cytoskeleton integrity (tau, MAP-2) and axonal transport (dynein, CRMP2) (Coleman, 2005; Knöferle et al., 2010). Optic nerve protein lysates were prepared from the two adjacent regions $1 \mathrm{~mm}$ proximal and distal to the crush site at $6 \mathrm{~h}$ after crush. As control, protein lysates were prepared from the corresponding regions of the native optic nerve. Expression levels of each protein in both proximal and distal parts were compared between lesioned optic nerves at $6 \mathrm{~h}$ after ONC and native optic nerves. If a protein is cleaved by the protease calpain, its expression levels are expected to decrease. 
The expression levels of the autophagy related proteins ATG5 and beclin-1 did not show any significant changes between both groups (Figure 3.5). Similarly, the levels of the protein tau were not significantly altered between both groups (Figure 3.6A,B). The levels of the microtubule-associated protein MAP-2 showed a significant decrease on the proximal side at $6 \mathrm{~h}$ after crush compared to control group. On the distal side of the crush there was also a clear trend towards decreased MAP-2 levels at $6 \mathrm{~h}$ after crush, which did, however, not reach significance (Figure 3.7). Levels of the axonal transport protein dynein (Vallee et al., 1989) were not changed (Figure 3.6C,D).

A
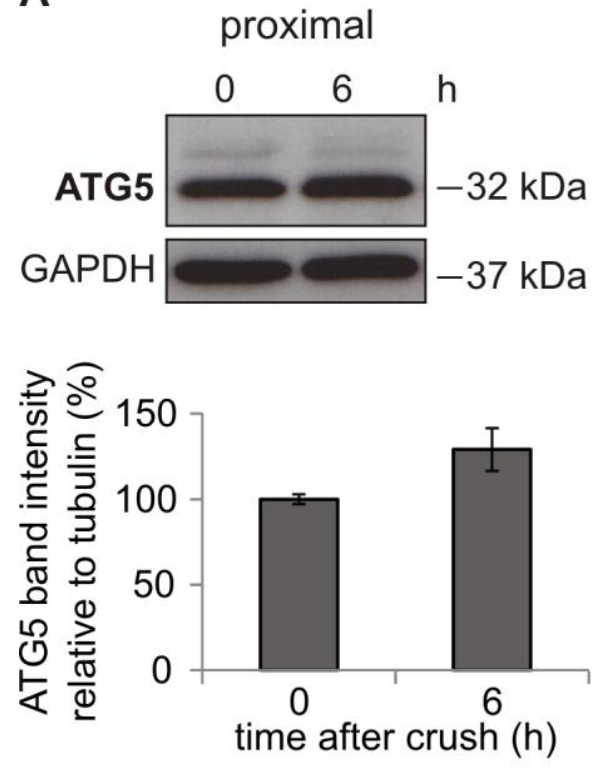

C

proximal
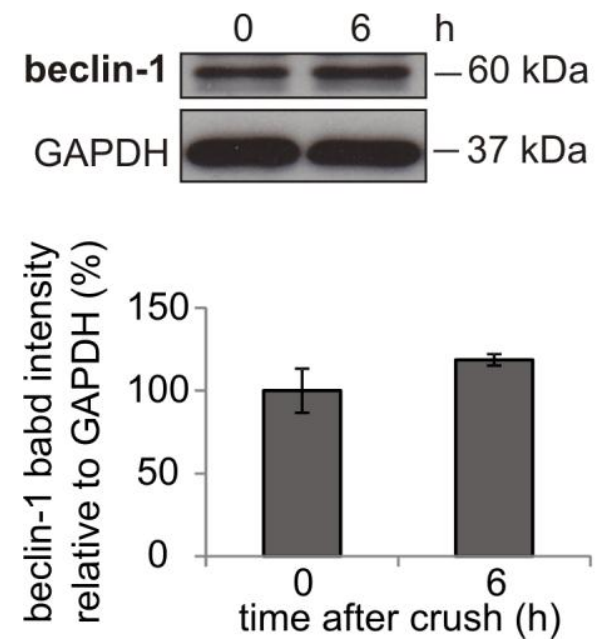

B
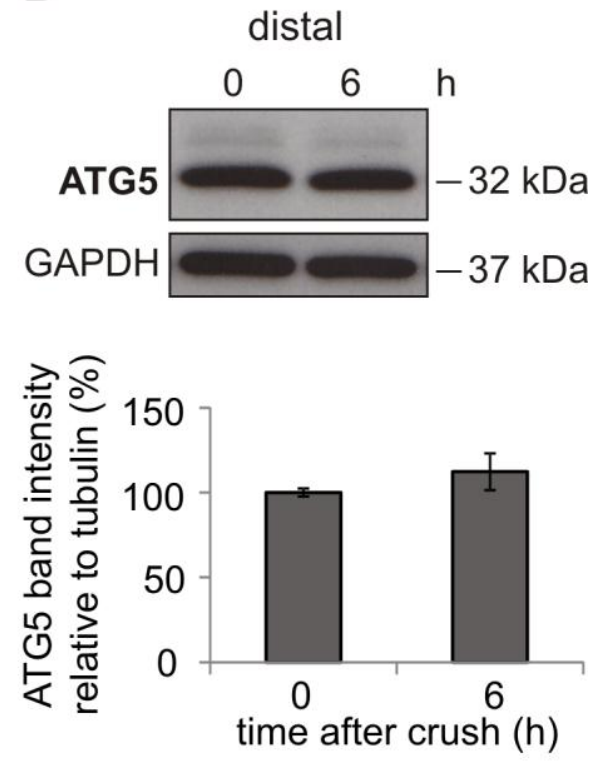

D
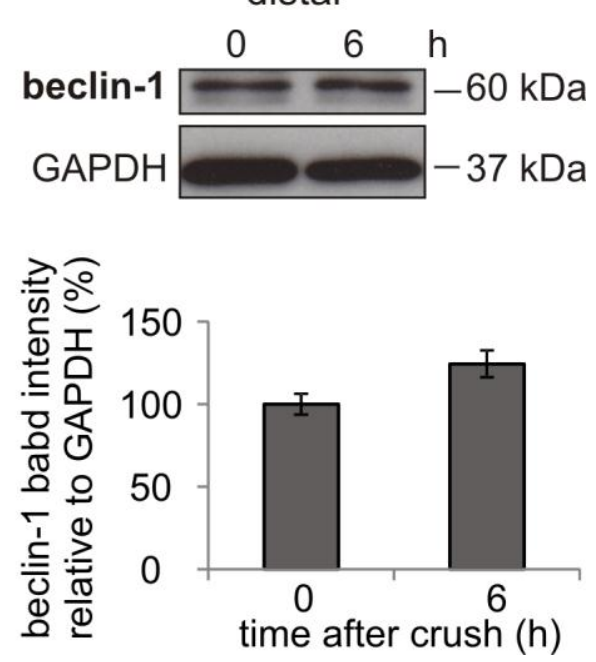
Figure 3.5 Immunoblot analyses of ATG5 and beclin-1 during acute axonal degeneration in vivo.

(A-D) Representative immunoblots of ATG5 and beclin-1 proximal and distal to the crush site in the upper panels. The protein lysates were made from native optic nerves ( $0 \mathrm{~h}$ after crush) and optic nerves $6 \mathrm{~h}$ after crush. The quantification of ATG5 and beclin-1 band intensity relative to GAPDH is shown at the bottom. 4 optic nerves are included in each group. Error bars represent the standard error of the mean (SEM).

A

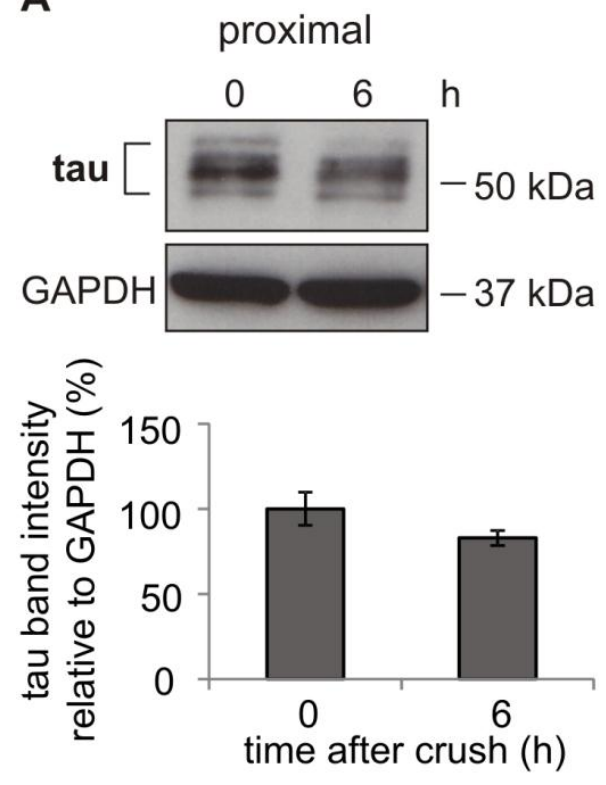

C
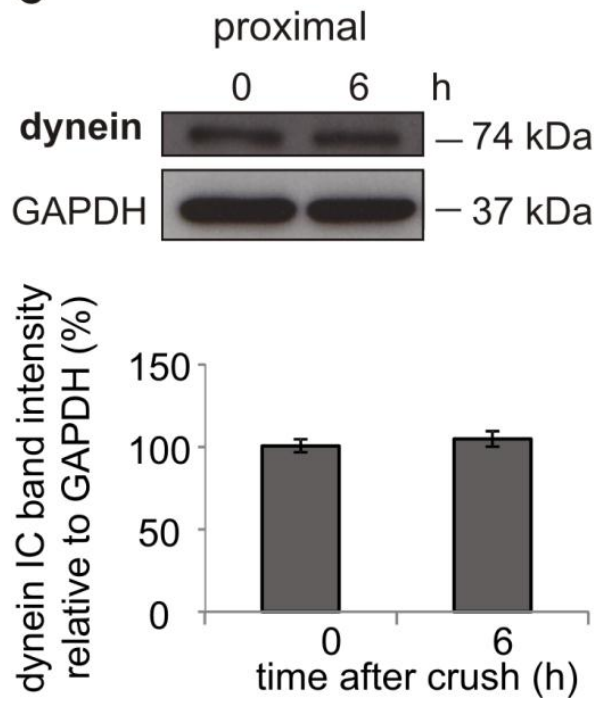

B
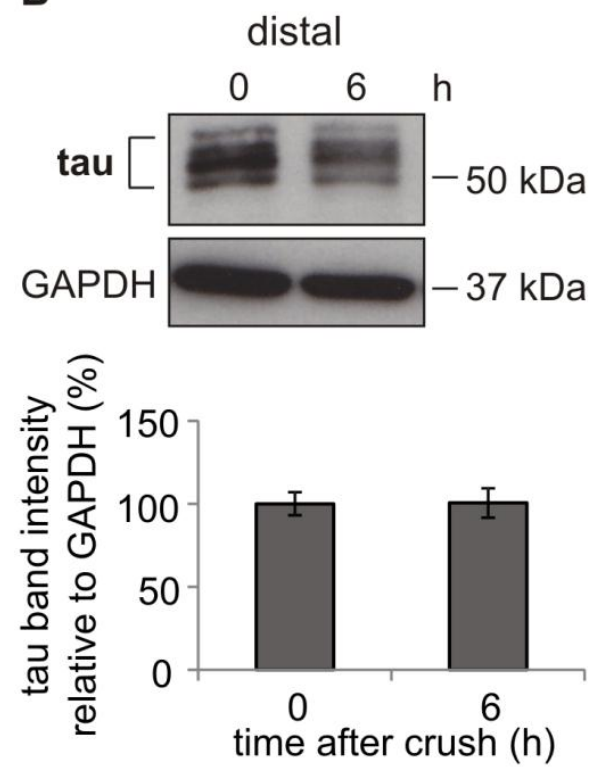

D
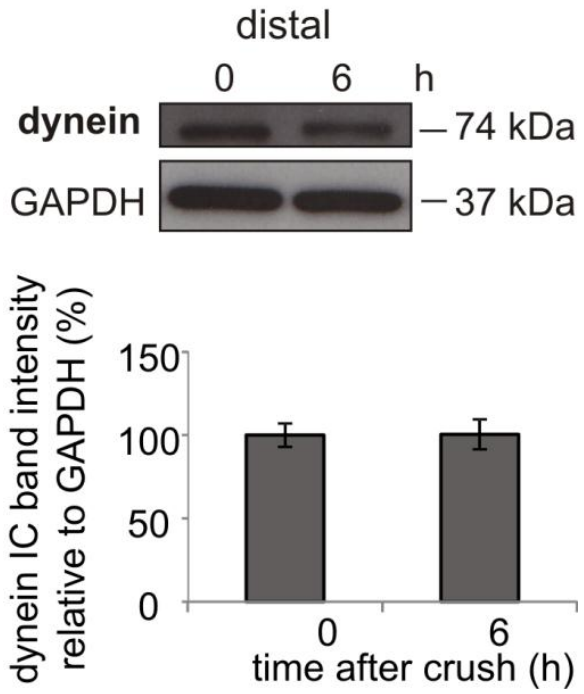

Figure 3.6 Immunoblot analyses of tau and dynein during acute axonal degeneration in vivo.

(A-D) Representative immunoblots of tau and dynein intermediate chain (dynein IC) proximal and distal to the crush site in the upper panels. The protein lysates were made from native optic nerves $(0 \mathrm{~h}$ after crush) 
and optic nerves $6 \mathrm{~h}$ after crush. The graphs at the bottom represent the quantification of the tau and dynein IC band intensity relative to GAPDH. 4 optic nerves are included in each group. Error bars represent the standard error of the mean (SEM).

A
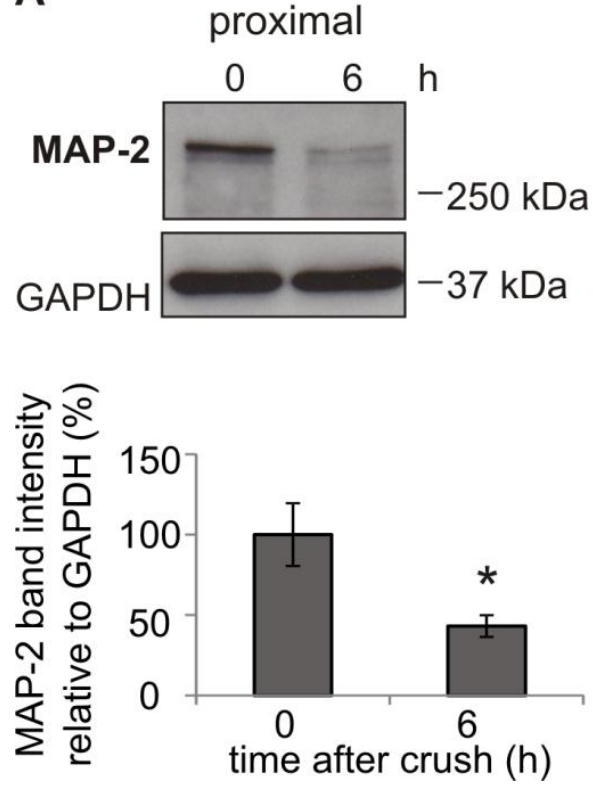

B
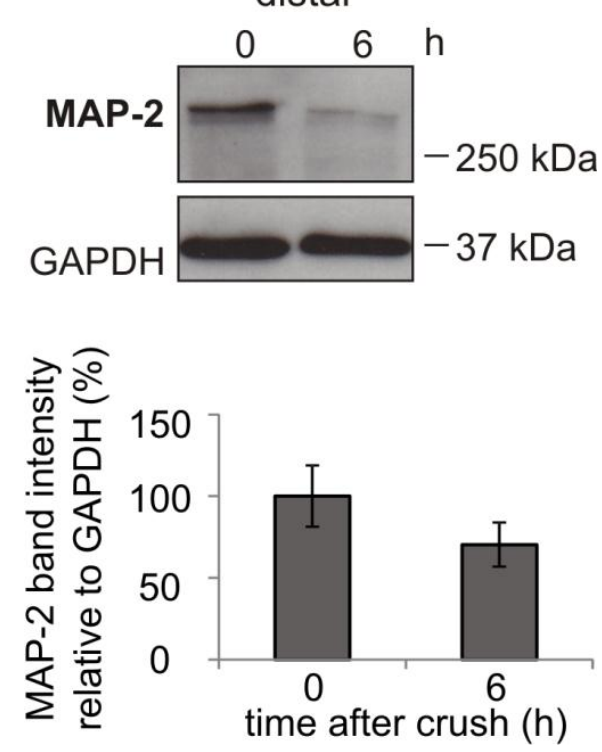

Figure 3.7 Immunoblot analysis of MAP-2 during acute axonal degeneration in vivo.

(A, B) Representative Western blots of MAP-2 proximal $(A)$ and distal $(B)$ to the crush site in the upper panels. The protein lysates were prepared from in the native optic nerves ( $0 \mathrm{~h}$ after crush) and optic nerves at $6 \mathrm{~h}$ after crush. At the bottom, the graphs show the quantification of MAP-2 band intensity relative to GAPDH. 4 optic nerves are included in each group. Error bars represent the standard error of the mean (SEM). ${ }^{*} p<0.05$ by independent samples t-test.

CRMP2 is a central protein linking axonal transport to the cytoskeleton (Arimura et al., 2005; Arimura, Hattori, et al., 2009; Fukata et al., 2002; Kimura et al., 2005). On the Western blot, the two isoforms CRMP2A and CRMP2B as well as a $58 \mathrm{kDa}$ calpain-derived cleavage product of CRMP2 can be discriminated. Interestingly, expression levels of this cleavage product of CRMP2 were significantly increased on both proximal and distal sides of the crush at $6 \mathrm{~h}$ after ONC (Figure 8A-B). Since the cleavage of CRMP2 was found to be the most 
prominent effect during AAD, we focused on the role of CRMP2 during AAD in the following experiments.

A

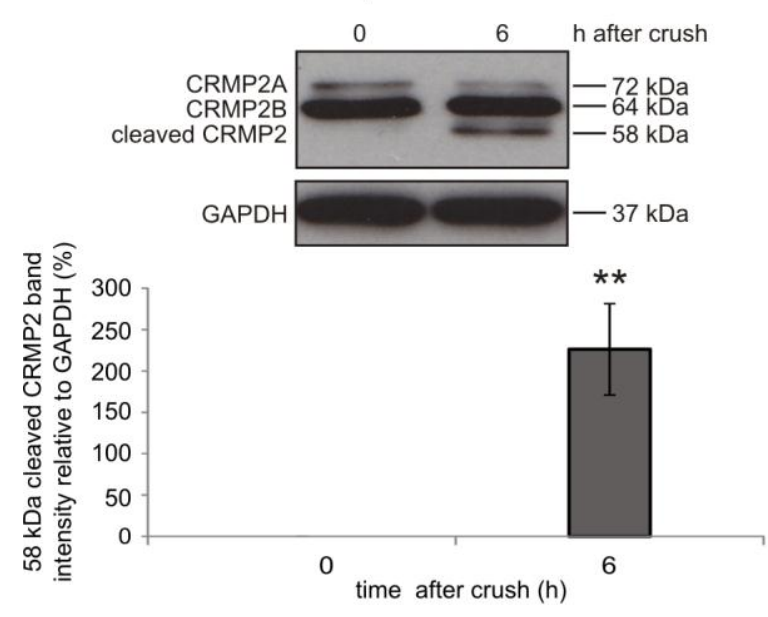

C

proximal
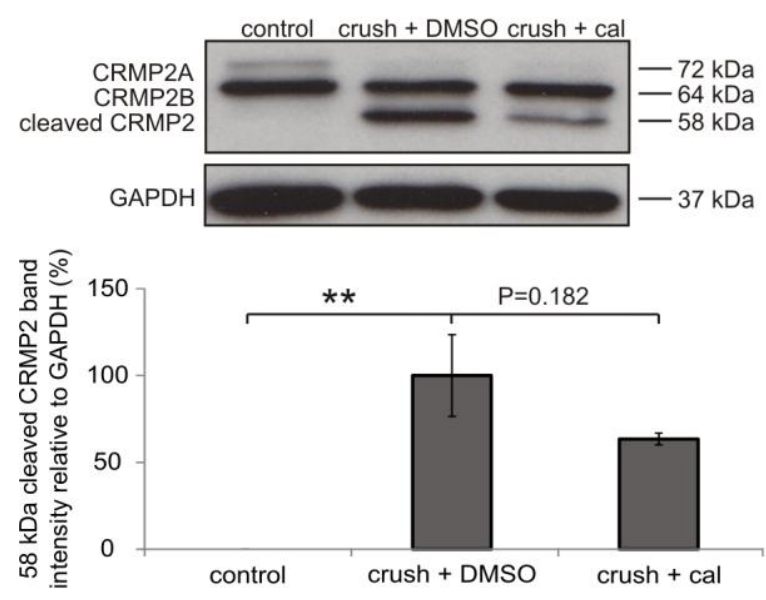

B

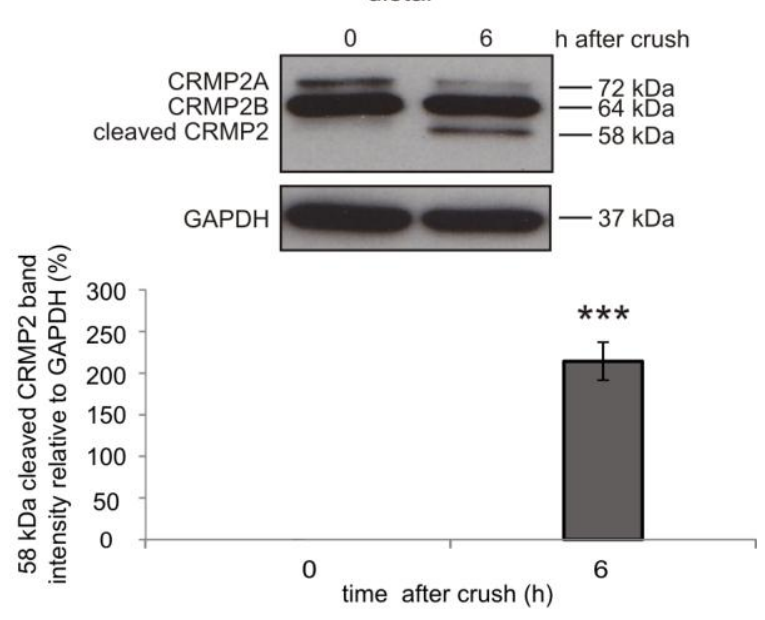

D

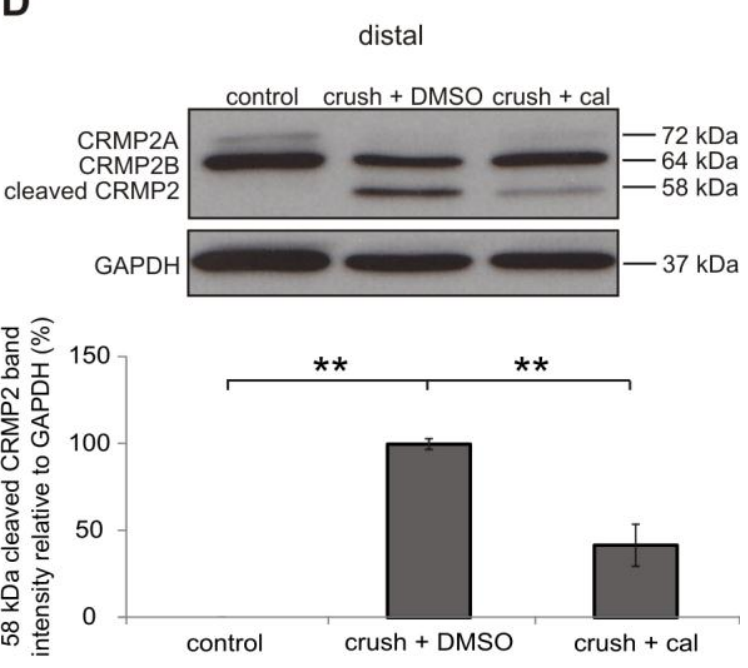

Figure 3.8 Calpain-mediated cleavage of CRMP2 during acute axonal degeneration in vivo.

(A, B) Representative CRMP2-immunoblots of optic nerve lysates proximal (A) and distal (B) to the crush site. The protein lysates were made from native optic nerves ( $0 \mathrm{~h}$ after crush) and optic nerves at $6 \mathrm{~h}$ after crush. The band intensity of cleaved CRMP2 was quantified and normalized to GAPDH, as displayed at the bottom. 4 optic nerves are included in each group. Error bars represent standard error mean (SEM). ${ }^{* *} p<$ $0.01, * * * p<0.001$ by independent samples t-test. 
(C, D) Representative immunoblots of CRMP2 proximal (A) and distal (B) to the crush site after calpeptin treatment in the upper panels. The protein lysates were prepared from native optic nerves, from optic nerves at $6 \mathrm{~h}$ after ONC pretreated with 7\% DMSO, and from optic nerves at $6 \mathrm{~h}$ after crush pretreated with $10 \mathrm{mM}$ calpeptin in 7\% DMSO (control, crush + DMSO \& crush + cal). Cal = Calpeptin. Below, the band intensity of cleaved CRMP2 was quantified relative to GAPDH. 4 optic nerves are included in each group. Error bars represent the standard error of the mean (SEM). Statistical significance is compared to crush + DMSO group: ${ }^{* *} p<0.01$ by one-way ANOVA and Dunnett's test.

To examine whether cleaved CRMP2 is indeed generated by calpain activation, we evaluated the effect of calpain inhibiton by calpeptin on the levels of cleaved CRMP2 at $6 \mathrm{~h}$ after ONC in vivo. Experimental groups included native optic nerves, optic nerves at $6 \mathrm{~h}$ after ONC pretreated with 7\% DMSO, and optic nerves at $6 \mathrm{~h}$ after crush pretreated with $10 \mathrm{mM}$ calpeptin in 7\% DMSO. Calpeptin or DMSO was injected intravitreally $2.5 \mathrm{~h}$ before ONC. Optic nerve protein lysates were prepared from the two adjacent regions $1 \mathrm{~mm}$ proximal and distal to the crush site at $6 \mathrm{~h}$ after ONC or from the corresponding regions of native optic nerves. In both proximal and distal parts, the increase of cleaved CRMP2 at $6 \mathrm{~h}$ after ONC was attenuated following calpeptin treatment compared to control (mean band intensity of the $58 \mathrm{kDa}$ CRMP2 cleavage product relative to GAPDH, proximal to the crush site: ONC + DMSO: $100 \pm 23.5 \%$; ONC + calpeptin: $63.4 \pm 3.3 \%$; distal to the crush site: ONC + DMSO: $100 \pm 3.1 \%$; ONC + calpeptin: $41.7 \pm 12.1$; without ONC: no cleaved CRMP2 detectable on both sides; $\mathrm{n}=3$ independent experiments) (Figure 3.8C,D).

The lesion-induced cleavage of CRMP2 by calpain was further confirmed in a scratch lesion model in primary cortical neurons in vitro (Tönges et al., 2011). The experimental groups included unlesioned cells pretreated with 0.1\% DMSO, lesioned cells pretreated with $0.1 \%$ DMSO, and lesioned cells pretreated with $50 \mu \mathrm{M}$ calpeptin in 0.1\% DMSO. On DIV8, DMSO or calpeptin was added to the culture medium at $1 \mathrm{~h}$ before scratch and cell lysates were collected at $6 \mathrm{~h}$ after scratch. Western blot analysis of spectrin and CRMP2 were performed. Compared to unlesioned cells, the levels of $145 \mathrm{kDa}$ BDP-spectrin and cleaved CRMP2 were significantly increased at $6 \mathrm{~h}$ after scratch in the lesioned cells. Pretreatment 
with the calpain-inhibitor calpeptin, however, significantly suppressed the increase of both $145 \mathrm{kDa}$ BDP-spectrin and cleaved CRMP2 at $6 \mathrm{~h}$ after scratch compared to the vehicle group (0.1\% DMSO) (Figure 3.9A-C).

Taken together, these results demonstrate that CRMP2 is cleaved by activated calpain during $A A D$ in vitro and in vivo.

A

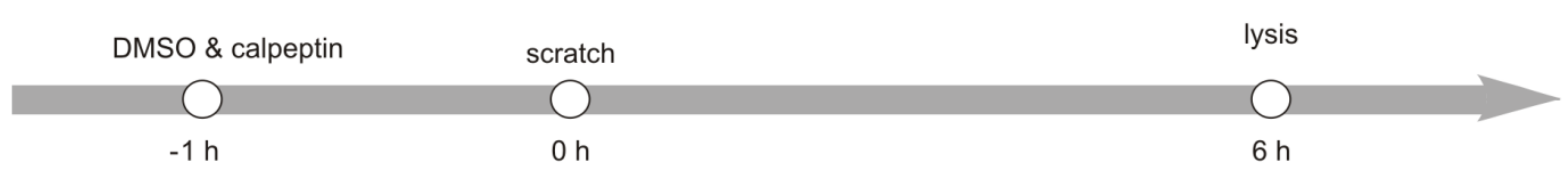

B

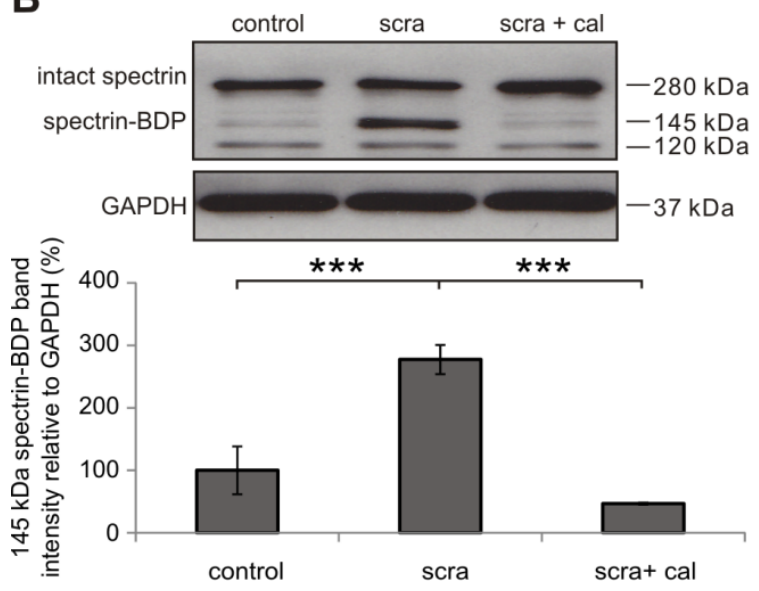

C

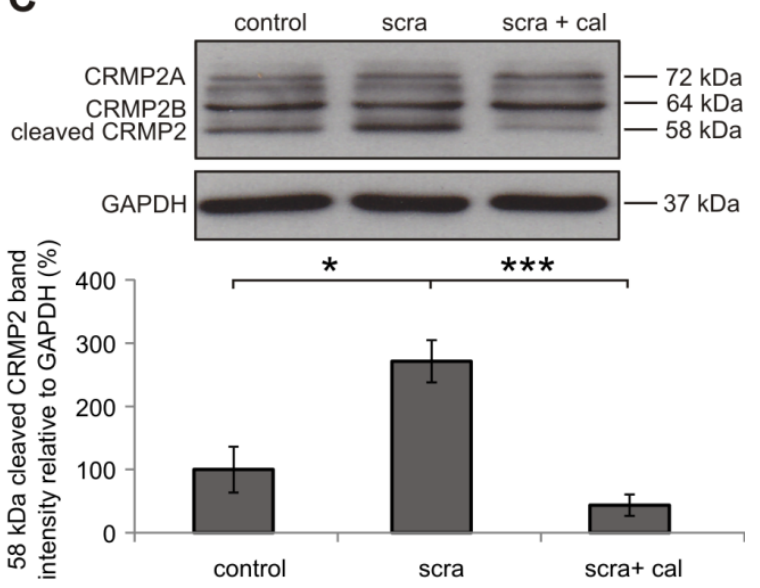

Figure 3.9 Calpain-mediated cleavage of CRMP2 in cortical neurons after scratch lesion in vitro.

(A) Experimental setup and time scale: On DIV8, $0.1 \%$ DMSO or $50 \mu \mathrm{M}$ calpeptin in $0.1 \%$ DMSO was applied to cortical neurons at $1 \mathrm{~h}$ before scratch lesion. Cell lysates were made and collected at $6 \mathrm{~h}$ after scratch.

(B, C) Representative immunoblots of spectrin (B) and CRMP2 (C) in cortical neurons. The experimental conditions include unscratched cells pretreated with $0.1 \%$ DMSO, scratched cells pretreated with $0.1 \%$ DMSO and scratched cells pretreated with calpeptin in $0.1 \%$ DMSO (control, scra + DMSO \& scra + cal). Cal $=$ calpeptin. Scra $=$ scratch. The graphs at the bottom show the quantification for the band intensity of $145 \mathrm{kDa}$ spectrin-BDP and cleaved CRMP2. GAPDH was used as loading control. 3 independent cultures are included per group. Error bars represent the standard error of the mean (SEM). ${ }^{*} p<0.05,{ }^{* * *} p<$ 0.001 by one-way ANOVA and Dunnett's test. 


\subsection{The role of CRMP2 in acute axonal degeneration}

\subsubsection{Establishment of an in vitro acute axonal degeneration model in the microfluidic} chamber system

Given that CRMP2 is cleaved by activated calpain during AAD, we hypothesized that an increase of intact CRMP2 protects axons from axonal degeneration. To examine this hypothesis, the microfluidic chamber system was used to establish an in vitro AAD model.

The experimental setup was optimized with regards to the following conditions. Each chamber was seeded with $3 \times 10^{5}$ cortical neurons after nucleofection with the plasmid CMV-EGFP. On DIV8, axons had grown across the microgrooves and extended into the axonal compartment. An axotomy was performed by vacuum aspiration through the axonal compartment for 5-10 s. This resulted in a specific lesion of the distal axons while the somata, dendrites and proximal axons were left unharmed due to the high fluidic resistance of the microgrooves. The axons were then imaged in a defined area spanning up to $400 \mu \mathrm{m}$ proximal from the lesion site. The imaging was performed before axotomy and at different time points after axotomy in a conditioned observation chamber.

Different from AAD in vivo, we did not observe a clear fragmentation of the axons up to $8 \mathrm{~h}$ after axotomy in vitro. The lesioned axons did, however, form axonal bulbs in a time-dependent manner (Figure 3.10). Since bulb formation is a well-known sign of axonal degeneration (Coleman, 2005), we quantified the number of newly formed bulbs along the axons after axotomy as a measure for axonal degeneration. There was a significant increase of the number of bulbs already at 5 min after lesion, which was followed by a small but significant decrease at $30 \mathrm{~min}$ and then again a continuous increase over the next 8 hours. 


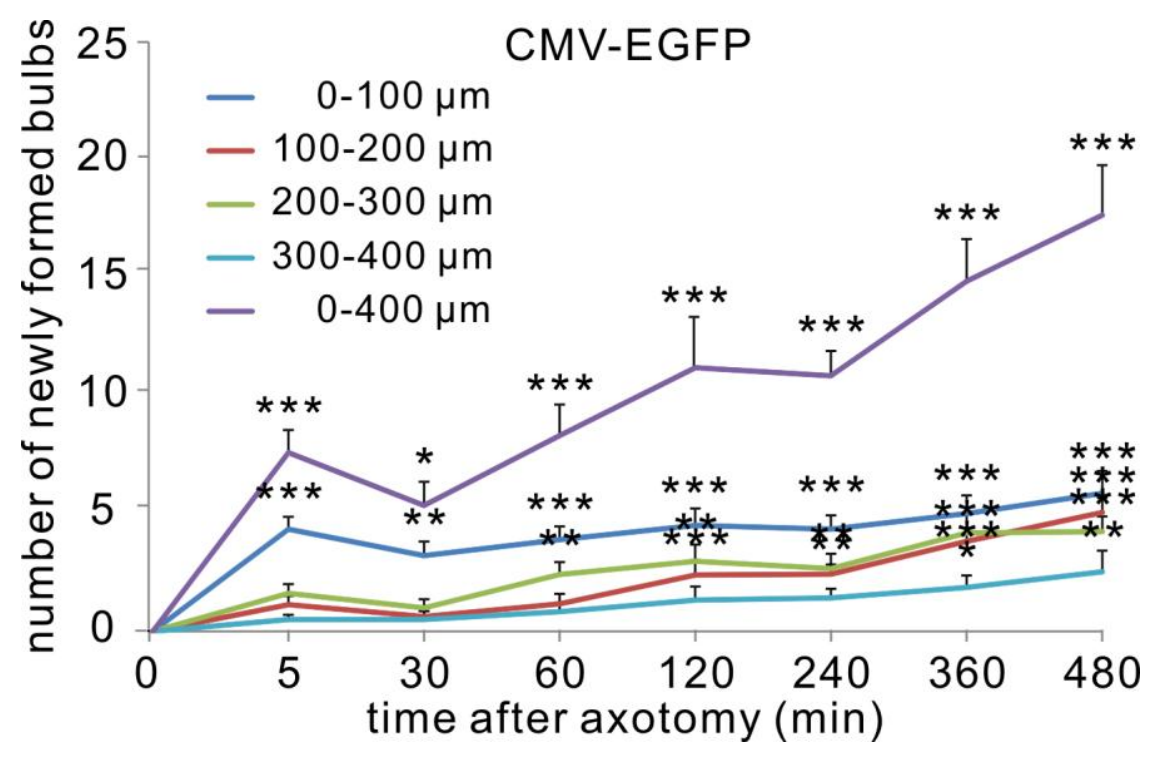

Figure 3.10 Imaging of bulb formation after axotomy of cortical neurons in the microfluidic chamber system in vitro.

The number of newly formed bulbs along the axons was quantified at the given distances to the lesion site. Error bars represent the standard error of the mean (SEM). 36, 36, 32, 36, 33, 29 and 21 axons are included at $0,5,30,60,120,240,360$ and 480 min after axotomy, respectively. Statistically significant differences are compared to before axotomy: ${ }^{*} p<0.05,{ }^{* *} p<0.01,{ }^{* * *} p<0.001$ by one-way ANOVA and Dunnett's test.

\subsubsection{Effects of CRMP2 overexpression on axonal degeneration after axotomy of cortical neurons in vitro}

We next evaluated the role of CRMP2 overexpression in the now established in vitro AAD model. To increase the levels of CRMP2, we first tested the drug tianeptine, which is a tricyclic antidepressant and was reported to rapidly increase the total levels of CRMP2 in cortical neurons (Hensley et al., 2011). To check whether tianeptine elicits the same effect in our conditions, it was added to the culture medium of cortical neurons in different concentrations on DIV8. After $24 \mathrm{~h}$, the cells were lysed for Western blot analysis of CRMP2. In the cultures treated with different concentrations of tianeptine, we could, however, not detect altered levels of intact CRMP2 compared to untreated cultures. Nevertheless, 
tianeptine induced increased levels of cleaved CRMP2. In a second approach based on our finding that the calpain-inhibitor calpeptin suppresses the cleavage of CRMP2, cortical neurons were therefore pretreated with calpeptin at $30 \mathrm{~min}$ before treatment with tianeptine (Figure 3.11A,B). As expected, calpeptin alone decreased the levels of cleaved CRMP2 (Figure 3.11A,B). It also significantly increased the levels of intact CRMP2, which was not observed elsewhere in this study. This might be due to the fact that the cells were incubated with calpeptin here for longer time than in all other experiments ( $24 \mathrm{~h}$ here versus $2.5 \mathrm{~h}$ in vivo and $6 \mathrm{~h}$ in vitro in other experiments). However, co-treatment with both calpeptin and tianeptine did not further increase the levels of intact CRMP2 compared to treatment with calpeptin alone (Figure 3.11A,B). These results thus did not support the use of tianeptine to increase intraneuronal levels of CRMP2.

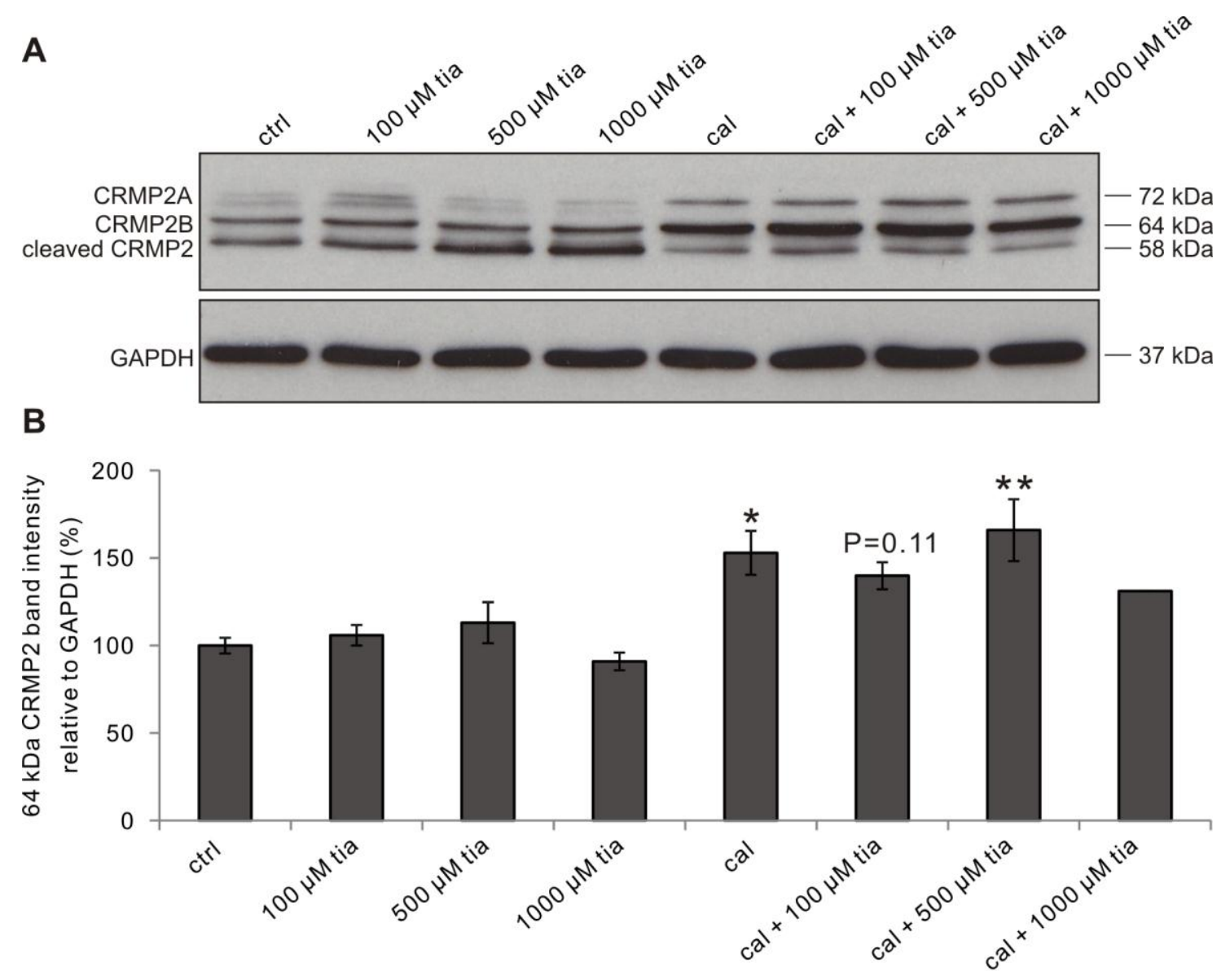

Figure 3.11 Effect of tianeptine on the levels of CRMP2 in cortical neurons in vitro. 
(A) A representative Western blot of cortical neuron whole cell protein lysates stained against CRMP2. The neurons were treated with tianeptine, calpeptine, or co-treated with both tianeptine and calpeptin. Tia $=$ Tianeptine. Cal $=$ Calpeptin. The band intensity of $64 \mathrm{kDa}$ intact CRMP2 was quantified relative to GAPDH in (B). Error bars represent the standard error of the mean (SEM). 3 cultures are included, ${ }^{*} p<$ $0.05, * * p<0.01$ by one-way ANOVA and Dunnett's test.

Since there are no other published pharmacological methods to increase the intraneuronal levels of CRMP2, we used nucleofection of neuronal cultures with the plasmid p.CMV-CRMP2-flag to overexpress CRMP2. This plasmid was provided by Mahnaz Moradi-Améli (Rogemond et al., 2008). To confirm the overexpression of the flag-tagged CRMP2 by this plasmid, Western blot analysis of flag was performed in cortical neurons transfected with the plasmid and revealed a flag-positive band with the molecular weight of CRMP2 (Figure 3.12A), thus suggesting the correct expression of CRMP2 by the plasmid. Since p.CMV-CRMP2-flag does not express fluorescent marker proteins, cortical neurons were regularly co-transfected with both p.CMV-EGFP and p.CMV-CRMP2-flag to identify the transfected axons. The co-transfection rate of the two plasmids was confirmed by immunocytochemistry against flag (Figure 3.12B). As control, cortical neurons were transfected with p.CMV-EGFP alone. In an area $400 \mu \mathrm{m}$ proximal to the lesion site, the number of newly formed bulbs was quantified in EGFP labeled axons in both groups. In both groups, the number of newly formed bulbs continued to increase over time. However, the number of bulbs was significantly decreased in the axons overexpressing CRMP2 at 30 min after axotomy compared to control group. This decrease was sustained up to $480 \mathrm{~min}$ after axotomy (at 30 min after axotomy: control: $5.3 \pm 1.0$; CRMP2: $1.2 \pm 1.0$; at $240 \mathrm{~min}$ after axotomy: control: $10.8 \pm 1.1$; CRMP2: $5.5 \pm 2.1$; at 480 min after axotomy: control: $17.7 \pm 2.1$; CRMP2: $10.5 \pm 2.5$ ) (Figure 3.12C,D).

Given that bulb formation is a correlate of axonal degeneration (Coleman, 2005), these results demonstrate that CRMP2 overexpression attenuates axonal degeneration after axotomy in vitro. 
A

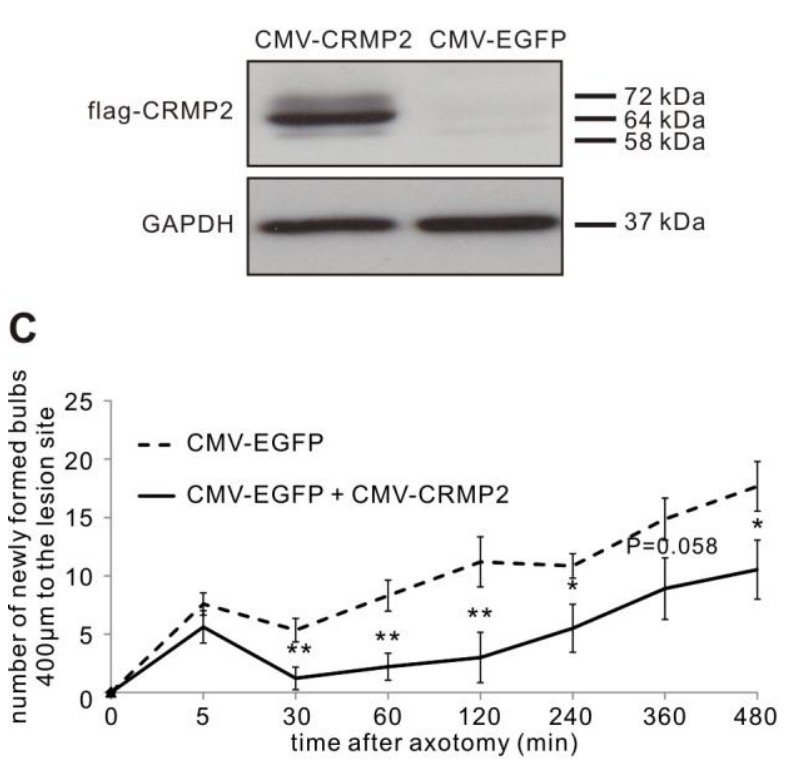

B

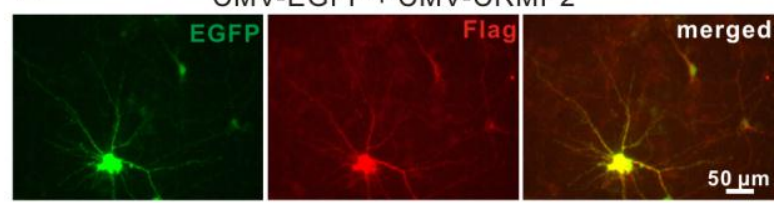

D

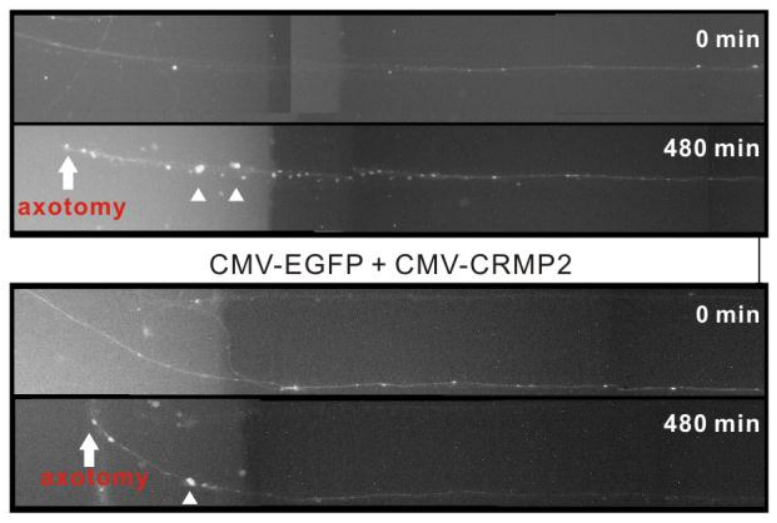

Figure 3.12 Effect of CRMP2 overexpression on bulb formation after axotomy of cortical neurons in vitro.

(A) Representative Western blot of cortical neuron whole cell protein lysates stained with an anti-flag antibody. The neurons were transfected with p.CMV-CRMP2-flag or p.CMV-EGFP as control. The blot confirms that p.CMV-CRMP2-flag mediates overexpression of CRMP2-flag.

(B) An anti-flag-immunostaining of cortical neurons co-transfected with p.CMV-CRMP2-flag and p.CMV-EGFP, confirming the successful co-transfection.

(C) The number of newly formed bulbs along the axons was quantified in the area $400 \mu \mathrm{m}$ proximal to the lesion at the given time points after axotomy. The axons were transfected with p.CMV-EGFP alone as control or co-transfected with both p.CMV-CRMP2-flag and p.CMV-EGFP. For p.CMV-EGFP alone, 36, 36, $32,36,36,33,29$ and 21 axons are included at 0, 5, 30, 60, 120, 240, 360 and 480 min after axotomy respectively. For cotransfection with both p.CMV-CRMP2-flag and p.CMV-EGFP, 29, 29, 23, 29, 28, 25, 21 and 19 axons are included at 0, 5, 30, 60, 120, 240, 360 and 480 min after axotomy respectively. Error bars represent the standard error of the mean (SEM). ${ }^{*} p<0.05,{ }^{* *} p<0.01$ by independent samples t-test. (D) The bulb formation in representative axons proximal to the lesion site before axotomy and at $480 \mathrm{~min}$ after axotomy. Arrows point to the site of axotomy. Arrowheads point to the bulbs. 


\subsubsection{Overexpression of CRMP2 mediated by the viral vector AAV.CRMP2}

In order to study the effects of CRMP2 overexpression in the rat optic nerve in vivo, we produced a viral vector to induce neuron-specific CRMP2 overexpression. To produce the viral vector, we cloned the plasmid p.AAV.hSyn-CRMP2-hSyn-mcherry based on the plasmid p.CMV-CRMP2-flag described above. Overexpression of CRMP2 mediated by this plasmid was first confirmed in HEK293 cells by Western blot of CRMP2 (Figure 3.13A) and further confirmed in cortical neurons by qRT-PCR analysis of human CRMP2 (Figure 3.13B). AAV (AAV.hSyn-CRMP2-mcherry) was then produced based on this plasmid and CRMP2 overexpression by the AAV confirmed in cortical neurons by qRT-PCR analysis (Figure 3.14A). Compared to transfection with the plasmid, CRMP2 expression was significantly stronger in cortical neurons transduced with AAV.hSyn-CRMP2-mcherry (as indicated by the appearance of the PCR product 10 cycles earlier than in the control plasmid transfected cells). In vivo, CRMP2 overexpression was confirmed in transduced optic nerves by qRT-PCR analysis (Figure 3.14B) and in retina lysates by Western blot analysis of CRMP2 (Figure 3.14C). 
A HEK 293 cells:

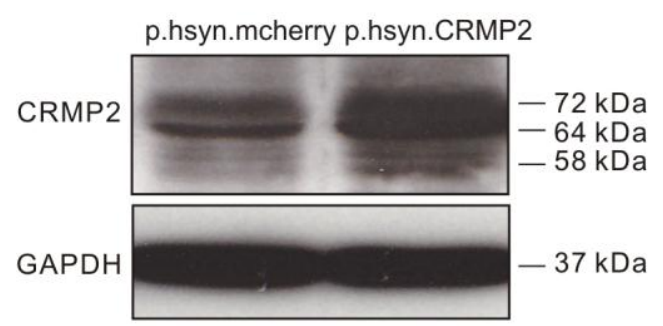

B cortical neurons:

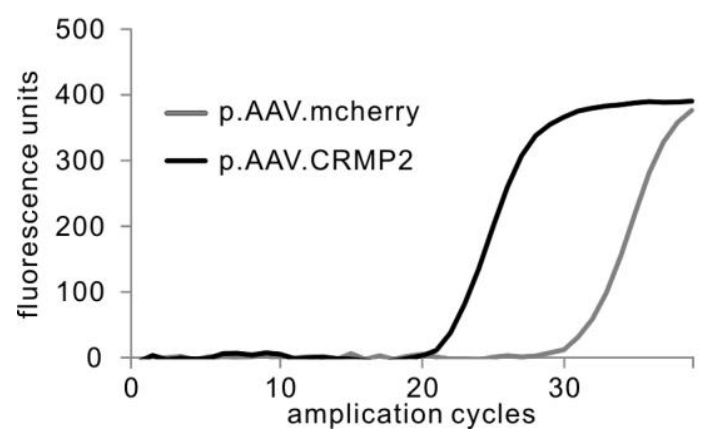

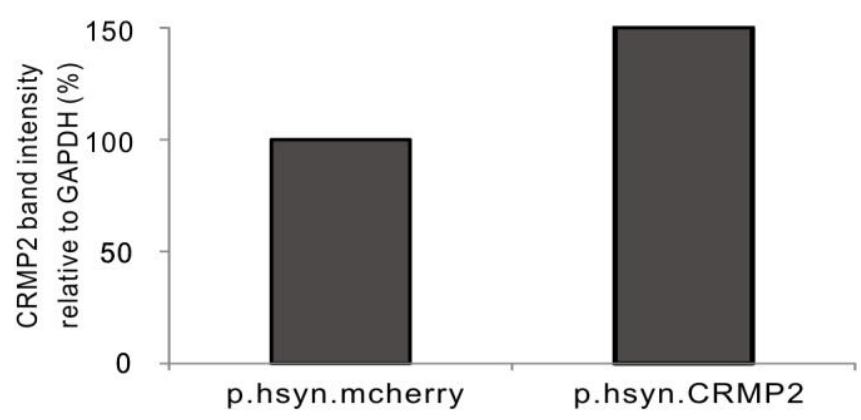

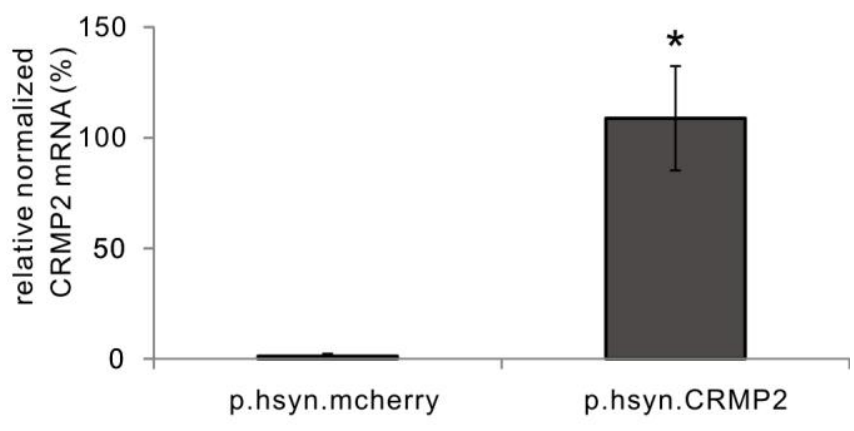

Figure 3.13 Testing of the CRMP2-expressing plasmid p.AAV.hSyn-CRMP2-hSyn-mcherry.

(A) A representative immunoblot of CRMP2 in HEK293 cells after transfection with p.AAV.mcherry or p.AAV.CRMP2. The right panel shows the quantification of CRMP2 band intensity relative to GAPDH.

(B) The results of a qRT-PCR analysis of rat cortical neurons using a human CRMP2 primer, which specifically detects the human CRMP2 expressed by the plasmid. The cells were transfected with p.AAV.mcherry or p.AAV.CRMP2. The left panel shows a representative amplification result ( $y$-axis: SYBR green fluorescence intensities; $x$-axis: number of PCR cycles). The right panel shows the quantification of human CRMP2 mRNA expression levels normalized to GAPDH mRNA expression. 3 cultures are included, ${ }^{*} p<0.05$ by independent samples t-test. 
A

cortical neurons:
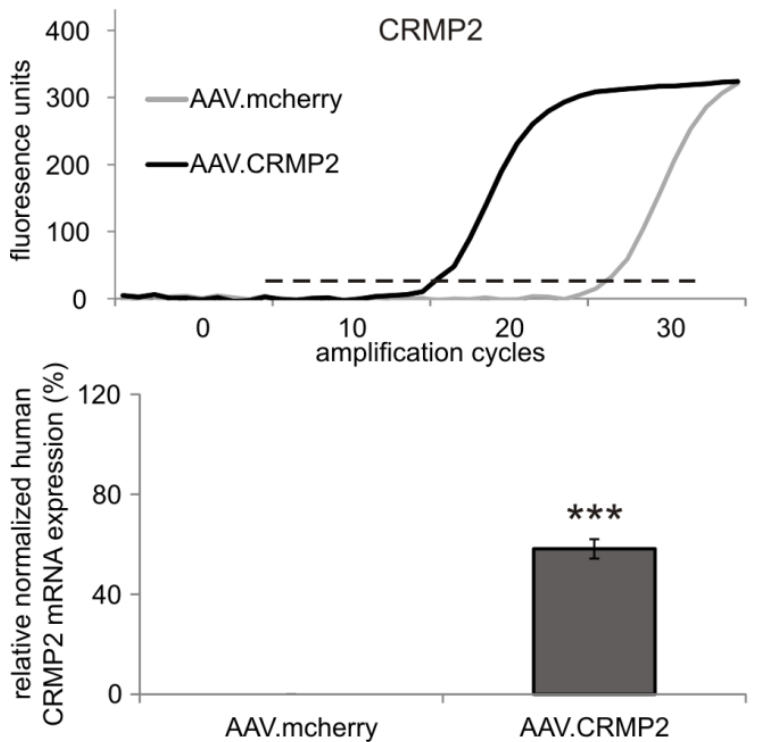

C
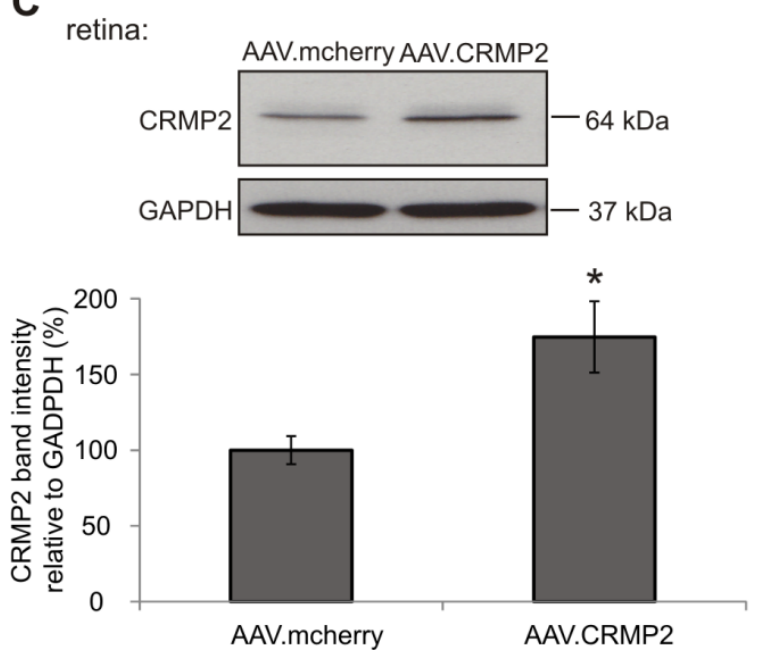

B optic nerve:
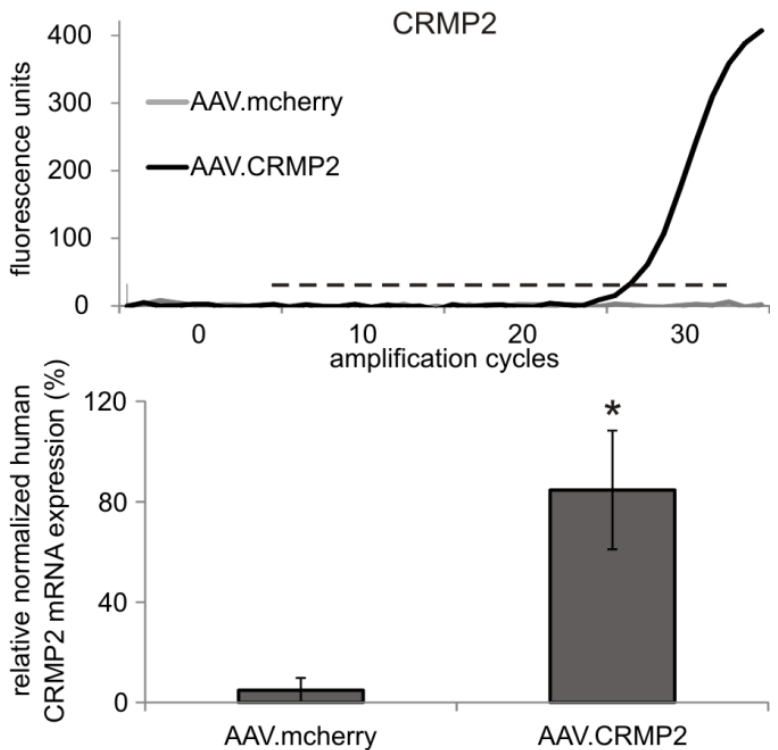

Figure 3.14 AAV-mediated overexpression of CRMP2 in vitro and in vivo.

(A, B) QRT-PCR analysis in cortical neurons and optic nerves using a human CRMP2 primer, which specifically detects the human CRMP2 expressed by the AAV. The upper panels show representative amplification graphs (y-axis: SYBR green fluorescence intensity; $x$-axis: number of PCR cycles), the lower panels show the relative human CRMP2 mRNA expression levels normalized to GAPDH mRNA expression. 3 independent cultures are included per group in (A). 3 optic nerves are included per group in (B). Error bars represent the standard error of the mean (SEM). ${ }^{* * *} p<0.001$ by independent samples t-test.

(C) Representative CRMP2 Western blot of retina lysates 4 weeks after intravitreal injections of 
AAV.CRMP2 or AAV.mcherry (upper panel). The lower panel shows the quantification of the CRMP2 band intensity relative to GAPDH. 3 retinas are included in each group. Error bars represent the standard error of the mean (SEM). ${ }^{*} p<0.05$ by independent samples t-test.

\subsubsection{Live imaging of crush-induced acute axonal degeneration in the rat optic nerve in vivo} after AAV-mediated CRMP2 overexpression

To study the effects of CRMP2 on AAD in vivo, RGC axons were transduced with AAV.hSyn-CRMP2-hSyn-mcherry overexpressing human CRMP2 and the fluorophore mcherry. As control, RGC axons were transduced with AAV.hSyn-mcherry-hSyn overexpressing only mcherry. Both AAV were injected intravitreally 4 weeks before imaging, resulting in sufficient fluorescent labeling of axons and good transduction rates. To study the time course of $A A D$, in vivo live imaging of the optic nerve was performed before crush and at various time points after crush. The AIR was quantified to evaluate axonal integrity during $A A D$. On the proximal side, CRMP2 overexpression almost completely blocked axonal fragmentation during the entire imaging period of $6 \mathrm{~h}$ compared to control group although some axonal bulbs were observed also in this group (Figure 3.15B,D). On the distal side, we did not detect any significant differences of the AIR between both groups but there was a trend towards a higher AIR (i.e. less degeneration) in the axons overexpressing CRMP2, especially at the later time-points (Figure 3.15C,E).

These results demonstrate that CRMP2 overexpression attenuates AAD on the proximal side after axonal lesion in the rat optic nerve in vivo. 
A
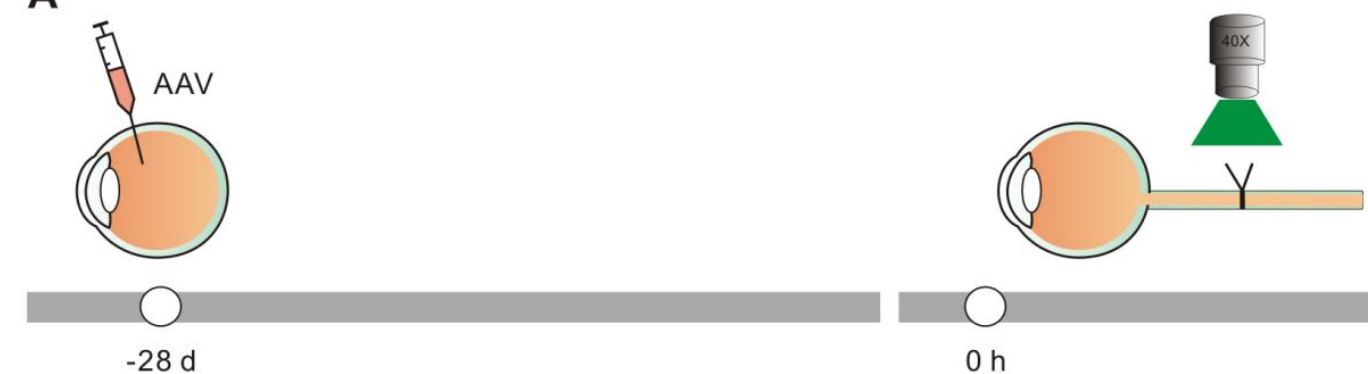

B

proximal

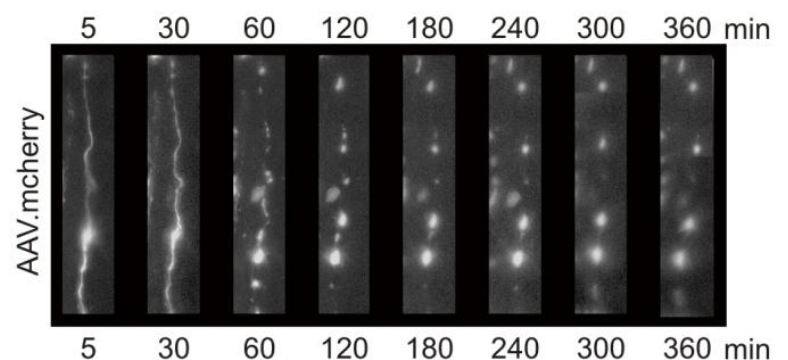

C

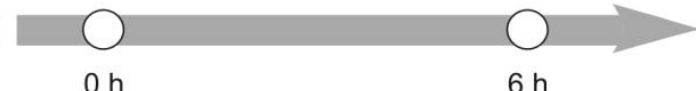

distal
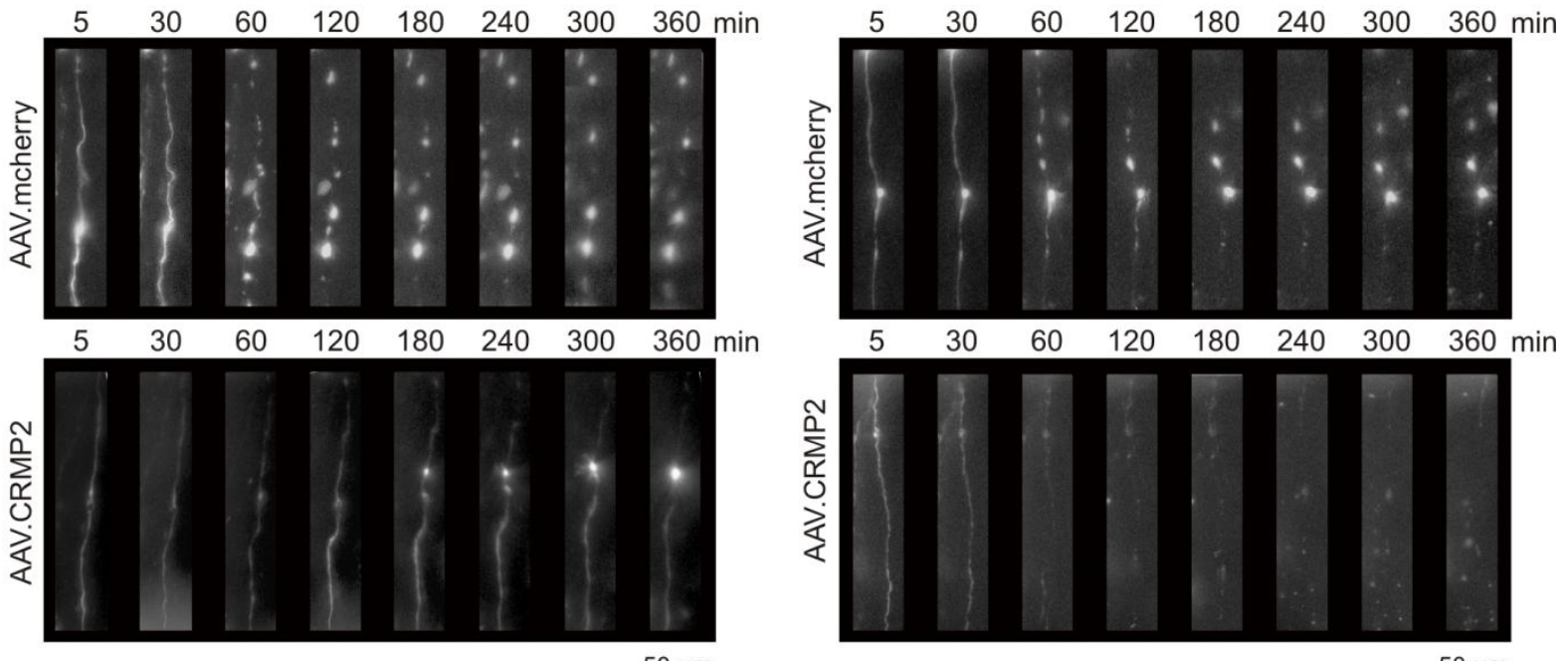

$\underline{50 \mu \mathrm{m}}$
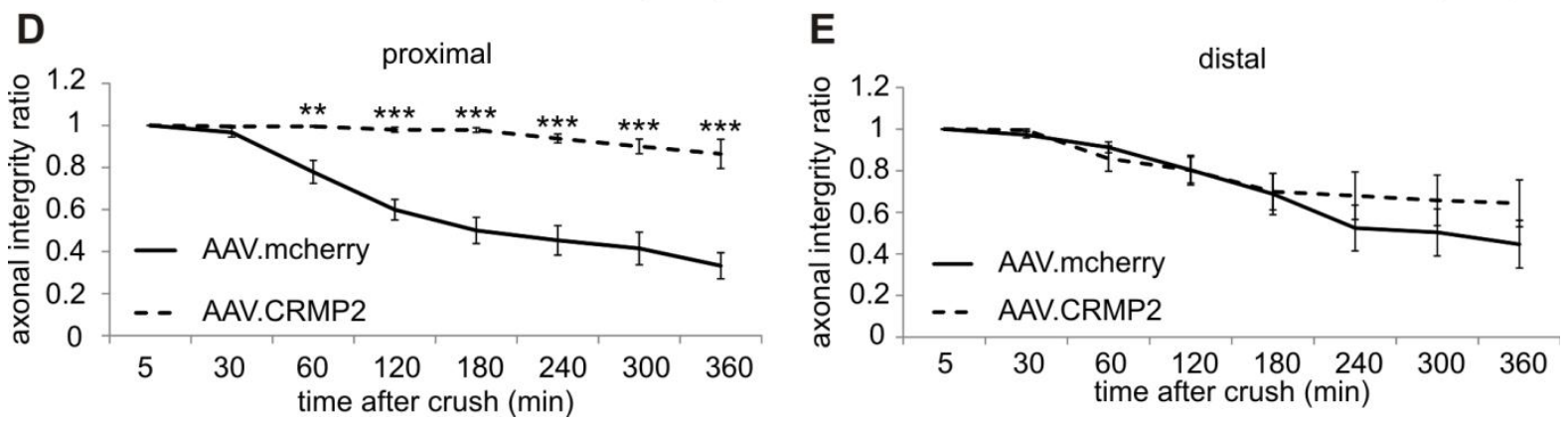

Figure 3.15 Time course of AAD in the optic nerve after AAV-mediated overexpression of CRMP2.

(A) Experimental setup and time-line: AAV.mcherry or AAV.CRMP2 was injected intravitreally to visualize the axons of retinal ganglion cells. After 4 weeks, the labeled axons were imaged $400 \mu \mathrm{m}$ proximal and distal to the crush site. Z-stack images were taken before ONC and over $6 \mathrm{~h}$ after ONC.

(B, C) Representative images of axonal changes proximal (B) and distal (C) to the crush site at the indicated time points after crush. The upper panels show axons transduced with AAV.mcherry as control, the lower panels represent axons transduced with AAV.CRMP2. 
(D, E) Quantification of axonal integrity ratios proximal (D) and distal (E) to the crush site at the indicated time points after crush. 5-7 rats are included per group. Error bars represent the standard error of the mean (SEM). Statistical significance is compared between the AAV.mcherry and AAV.CRMP2 groups at each time-point: ${ }^{* *} p<0.01,{ }^{* * *} p<0.001$ by independent samples t-test.

\subsubsection{Effects of CRMP2 overexpression on mitochondrial transport after axotomy}

Axonal bulbs are observed already at an early stage of AAD and are characterized by an accumulation of different organelles including mitochondria (Knöferle et al., 2010). A possible explanation for the formation of axonal bulbs is an impairment of axonal transport during AAD. To examine this hypothesis, we imaged the kinetics of mitochondrial transport in cortical neurons after axotomy. The imaging was performed in the microfluidic chambers system, which allowed us to image mitochondrial transport specifically in the axons and to differentiate between anterograde and retrograde transport. To visualize mitochondria, primary cortical neurons were transduced with AAV.hSyn-mito-RFP, expressing red fluorescent protein (RFP) specifically targeted to mitochondria. On DIV8, mitochondrial transport was imaged in single axons before axotomy and at different time points after axotomy. We quantified mitochondrial transport by kymographs in the region $100 \mu \mathrm{m}$ proximal to the lesion site since this was the area most affected by AAD after axotomy (see above). The mitochondria with a speed of at least $0.07 \mu \mathrm{m} / \mathrm{s}$ were defined as motile mitochondria. Compared to before axotomy $(27.3 \% \pm 1.7 \%)$, the percentage of motile mitochondria was significantly decreased within $60 \mathrm{~min}$ after axotomy $(5 \mathrm{~min}: 8.3 \% \pm 1.5 \%$; $30 \mathrm{~min}: 8.0 \% \pm 2.5 \%$; $60 \mathrm{~min}: 14.8 \% \pm 3.1 \%$ ) (Figure 3.16B,C). However, at $2 \mathrm{~h}$ after axotomy $(24.5 \% \pm 2.8 \%)$, the percentage returned to normal levels (Figure 3.16B,C). Among the motile mitochondria, we quantified the transport speed in both anterograde and retrograde direction. At $5 \mathrm{~min}$ after axotomy, the anterograde transport speed of motile mitochondria decreased significantly compared to before axotomy but it recovered to normal levels already at $30 \mathrm{~min}$ after axotomy (before axotomy: $0.32 \pm 0.02 \mu \mathrm{m} / \mathrm{s} ; 5 \mathrm{~min}$ after axotomy: $0.20 \pm 0.02 \mu \mathrm{m} / \mathrm{s} ; 30 \mathrm{~min}$ after axotomy: $0.37 \pm 0.05 \mu \mathrm{m} / \mathrm{s}$ ) (Figure 3.16B,D). The retrograde speed of motile mitochondria did not show any significant changes at any of the analyzed 
time points (Figure 3.16B,D). These results show that axonal transport of mitochondria is impaired early but transiently during AAD after axotomy.

A

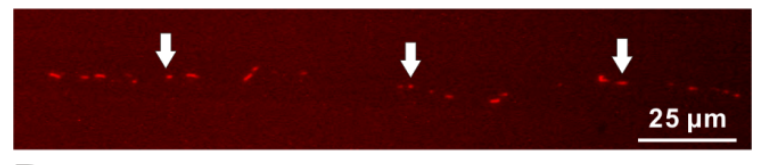

B

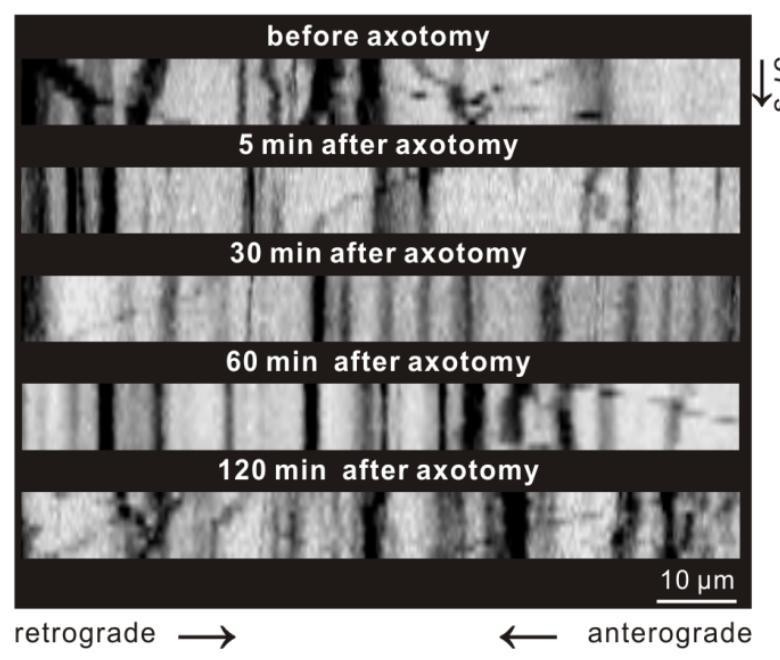

C

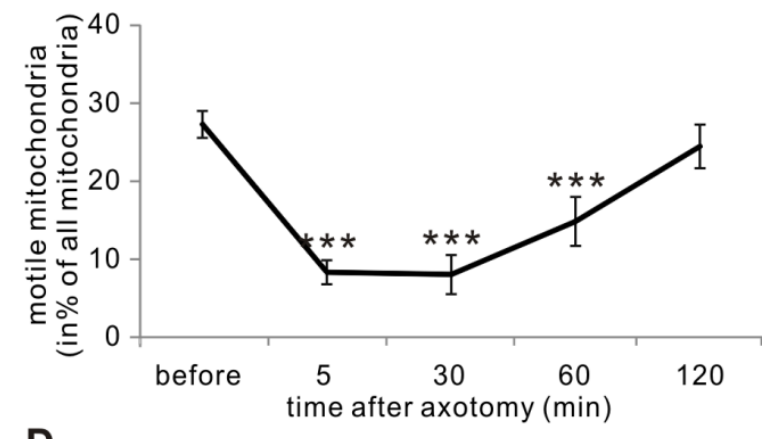

D

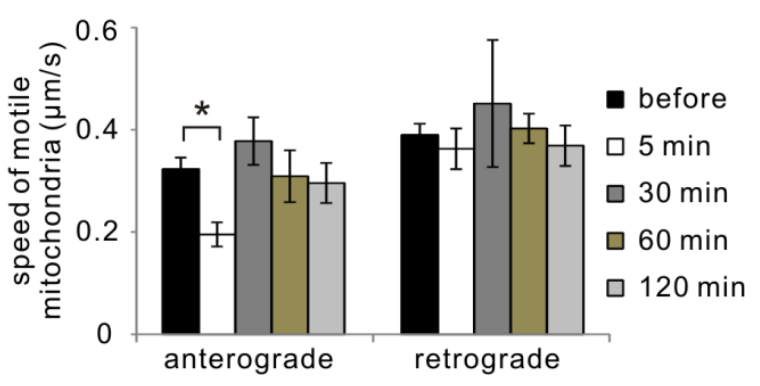

Figure 3.16. Live imaging of mitochondrial transport after axotomy of cortical neurons in the microfluidic chamber system in vitro.

(A) Labeled mitochondria along an axon of a cortical neuron transduced with AAV.mito-RFP. Arrows point to mitochondria.

(B) Representative kymographs of mitochondrial transport before axotomy and at the indicated time points after axotomy. The kymographs were made along the $100 \mu \mathrm{m}$ axonal segments proximal to the lesion site within $37 \mathrm{~s}$ (y-axis).

(C, D) Quantification of mitochondrial transport along the $100 \mu \mathrm{m}$ axon segments proximal to the lesion site. The percentage of motile mitochondria is displayed in (C). The anterograde and retrograde speed of motile mitochondria are represented in (D). 18-20 axons are included for each time point and each group. Error bars represent the standard error of the mean (SEM). Statistical significance is compared to before axotomy: ${ }^{*} p<0.05,{ }^{* * *} p<0.001$ by one-way ANOVA and Dunnett's test. 
CRMP2 contributes to the regulation of axonal transport by adapting the motor protein kinesin-1 to transport packets (Arimura, Kimura, et al., 2009; Kimura et al., 2005). Based on our findings that CRMP2 delays axonal degeneration and bulb formation during AAD, we hypothesized that alterations in CRMP2-levels affect mitochondrial transport in AAD. Therefore we investigated the effects of CRMP2 overexpression on mitochondrial transport in the microfluidic chamber system after axotomy. To overexpress CRMP2, cortical neurons were transduced with AAV.hSyn-CRMP2-hSyn-mcherry. As control, AAV.hSyn-mcherry-hSyn was employed. As AAV.hSyn-CRMP2-hsyn-mcherry expresses a red fluorescent protein and therefore the AAV.mito-RFP could not be used in this experiment, we added mitotracker green to label mitochondria. This allowed us to image mitochondrial transport specifically in the axons overexpressing CRMP2 (labeled red). Based on our results that mitochondrial transport was impaired within the first hour after axotomy, live imaging of mitochondrial transport was performed before axotomy and within $1 \mathrm{~h}$ after axotomy. In the unlesioned axons, neither the percentage of motile mitochondria (AAV.hSyn-mcherry-hSyn: $25.3 \% \pm$ 2.0\%; AAV.hSyn-CRMP2-hSyn-mcherry: $29.8 \% \pm 2.2 \%$ ) nor the speed of motile mitochondria (anterograde speed: AAV.hSyn-mcherry-hSyn: $0.33 \pm 0.04 \mu \mathrm{m} / \mathrm{s} ;$ AAV.hSyn-CRMP2-hSynmcherry: $0.28 \pm 0.02 \mu \mathrm{m} / \mathrm{s}$; retrograde speed: AAV.hSyn-mcherry-hSyn: $0.41 \pm 0.03 \mu \mathrm{m} / \mathrm{s}$; AAV.hSyn-CRMP2-hSyn-mcherry: $0.41 \pm 0.05 \mu \mathrm{m} / \mathrm{s}$ ) showed any significant differences between AAV.hSyn-mcherry-hSyn or AAV.hSyn-CRMP2-hSyn-mcherry-treated cultures (Figure 3.17). In the lesioned axons, however, the decreased percentage of motile mitochondria at $30 \mathrm{~min}$ after axotomy was almost completely rescued after overexpression of CRMP2 (AAV.hSyn-mcherry-hSyn: $9.4 \pm 2.3 \%$ of all mitochondria; AAV.hSyn-CRMP2-hsynmcherry: $23.2 \pm 3.3 \%$ of all mitochondria). However, at $60 \mathrm{~min}$ after axotomy, the percentage of motile mitochondria was not significantly different anymore between both groups. Overexpression of CRMP2 did not affect the speed of motile mitochondria at all analyzed time-points after axotomy. Thus, these results show that CRMP2 overexpression temporally rescues mitochondrial transport after axotomy in vitro. 
A

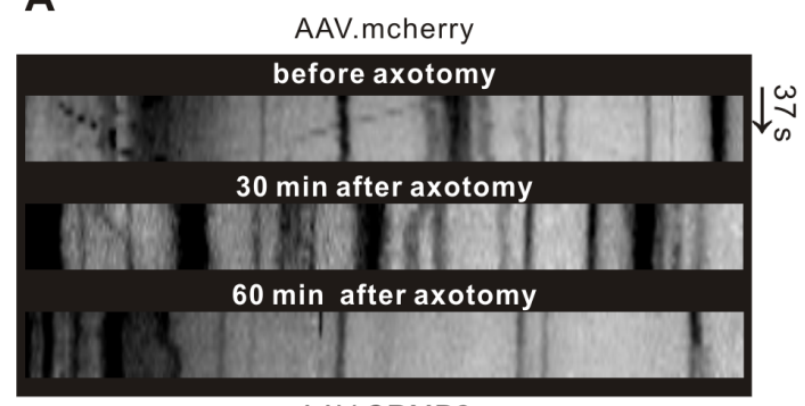

AAV.CRMP2

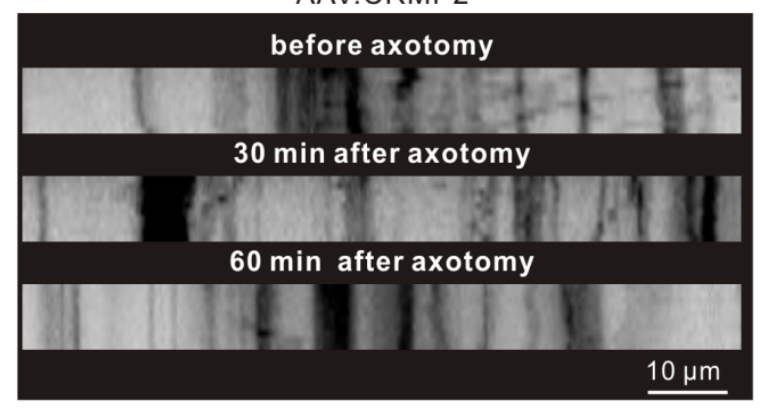

retrograde $\longrightarrow$
B
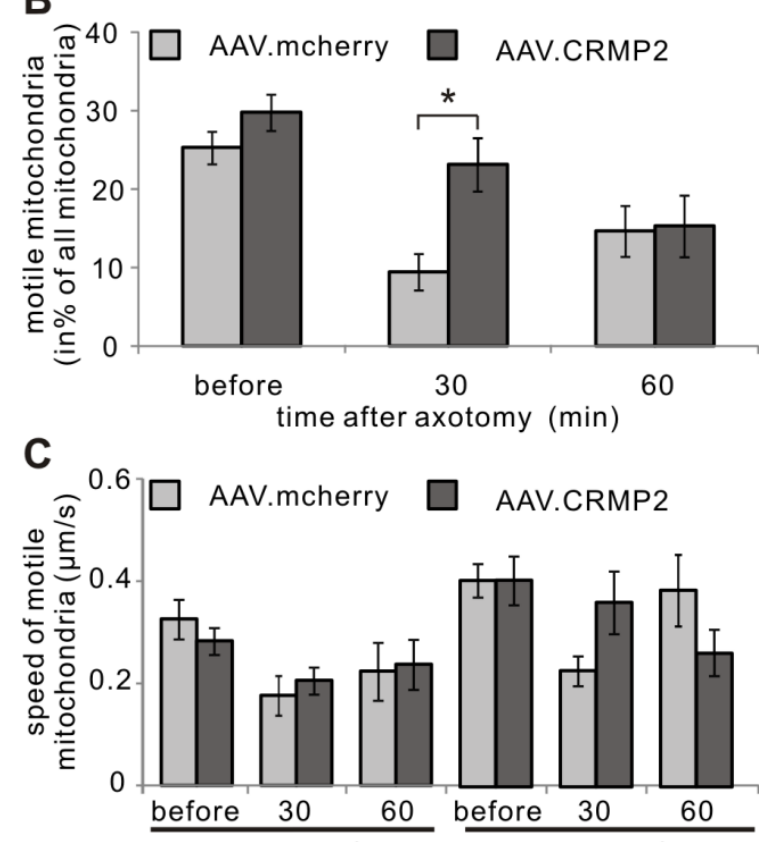

anterograde

Figure 3.17 Effect of CRMP2 overexpression on mitochondrial transport after axotomy of cortical neurons in the microfluidic chamber system in vitro.

(A) Representative kymographs of mitochondrial transport within $37 \mathrm{~s}$ (y-axis) before axotomy and at the indicated time points after axotomy. The kymographs were made along the $100 \mu \mathrm{m}$ axonal segments proximal to the lesion site. The upper panel shows representative kymographs from an axon transduced with AAV.mcherry, the lower panel from an axon transduced with AAV.CRMP2.

(B, C) Quantification of mitochondrial transport along the $100 \mu \mathrm{m}$ axonal segments proximal to the lesion site. The percentage of motile mitochondria is displayed in (B). The anterograde and retrograde speed of motile mitochondria are represented in (C). $\mathrm{N} \geq 14$ axons per group. Error bars represent the standard error of the mean (SEM). ${ }^{*} p<0.05$ by independent samples t-test.

\subsection{Proteomics analysis of acute axonal degeneration in the optic nerve}

To identify further molecular targets and CRMP2-interacting partners, which contribute to $A A D$, a proteomics analysis of the rat optic nerve during $A A D$ was performed. Optic nerve lysates were prepared from two areas comprising $1 \mathrm{~mm}$ proximal or distal from the crush site 
or the corresponding areas of native optic nerves. In order to reduce the variations due to inter-animal variability, the protein lysates of four independent optic nerves were pooled into one sample per group. In total, 2685 proteins were identified in the optic nerve lysates. The expression levels of 135 proteins were differentially expressed between the groups. For all these 135 regulated proteins, a STRING database (v10 for rat) search (Szklarczyk et al., 2014) was performed to check for reported interactions with CRMP2. 12 CRMP2-interacting proteins were identified (Figure 3.18 and Table 3.1). The expression levels of these proteins in proximal or distal part at $6 \mathrm{~h}$ after ONC were compared to control group. When the expression ratio was more than 1.3 or less than -1.3 , the levels of these CRMP2-interacting partners were considered changed at $6 \mathrm{~h}$ after ONC relative to control group. Among these 12 CRMP2-interacting partners, the ones related to the cytoskeleton were increased in expression during AAD compared to control group. These proteins included the alpha-II and beta-Il subunits of spectrin on both proximal and distal sides of the lesion, the actin-binding protein alpha-actinin 4 (ACTN4) (Otey CA, 2004) on the proximal side of the lesion, as well as the cytoskeleton regulators septin 2 (SEPT2) (Spiliotis, 2010) and cell division control protein 42 (CDC42) (Etienne-Manneville, 2004) on the distal side of the lesion. The mitochondrial enzyme malate dehydrogenase $2(\mathrm{MDH} 2)$ was increased on both proximal and distal sides of the lesion. On the other hand, the motor protein kinesin-like protein 1C (KIF1C) (Hirokawa et al., 2009), the activator of the ERK/MAPK pathway astrocytic phosphoprotein 15 (PEA-15) (Formstecher et al., 2001; Ramos et al., 2000), and the metabolic enzyme omega-amidase (NIT2) were decreased on both proximal and distal sides of the lesion. The levels of aspartate transaminase (GOT1) and G protein beta subunit 1 (GNB1) were decreased only on the proximal side of the lesion. The expression levels of 14-3-3 protein epsilon (YWHAE) were decreased on the proximal side but increased on the distal side. 
Table 3.1 List of proteins linked to CRMP2 with altered expression during acute axonal degeneration in the rat optic nerve identified by proteomics analysis.

\begin{tabular}{|l|l|l|l|l|}
\hline uniProtID & protein & function & $\begin{array}{l}\text { ratio } \\
\text { (proximal) }\end{array}$ & $\begin{array}{l}\text { ratio } \\
\text { (distal) }\end{array}$ \\
\hline P04636 & MDH2 & mitochondrial protein & 2.0 & 1.8 \\
\hline P16086 & SPTAN1 & cytoskeletal protein & 2.3 & 1.4 \\
\hline G3V6S0 & SPTBN1 & cytoskeletal protein & 3.1 & 1.9 \\
\hline Q9QXQ0 & ACTN4 & regulation of actin cytoskeleton & 1.9 & -1.1 \\
\hline Q91Y81 & SEPT2 & regulation of actin and tubulin & -1.1 & 1.5 \\
\hline Q8CFN2 & CDC42 & regulation of actin and tubulin & -1.1 & 2.2 \\
\hline P62260 & YWHAE & regulation of intermediate filaments & -1.4 & 1.5 \\
\hline P54311 & GNB1 & signal transduction & -1.6 & 1.0 \\
\hline P13221 & GOT1 & glutamate metabolism & -1.6 & -1.1 \\
\hline Q5U318 & PEA-15 & activation of the ERK/MAPK pathway & -3.3 & -2.1 \\
\hline P54311 & GNB1 & signal transduction & -1.6 & 1.0 \\
\hline Q497B0 & NIT2 & glutamate and asparagine metabolism & -1.7 & -1.3 \\
\hline F1M9C8 & KIF1C & motor protein of axonal transport & -3.7 & -3.7 \\
\hline
\end{tabular}

The given proteins are CRMP2-interacting partners identified by a STRING database (v10 for rat) search. Their expression levels are significantly regulated at $6 \mathrm{~h}$ after ONC in the proximal and/or distal part to the lesion compared to control. The fold changes relative to control are shown on the right side of the table. Each value represents the mean of two technical replicates for a pooled sample. Each pooled sample includes 4 independent optic nerves. Statistical significance is according to ANOVA analysis using a Benjamini-Hochberg-corrected p-value of 0.05 to judge significance. The protein levels are considered regulated when the fold change is more than 1.3 or less than -1.3 . 

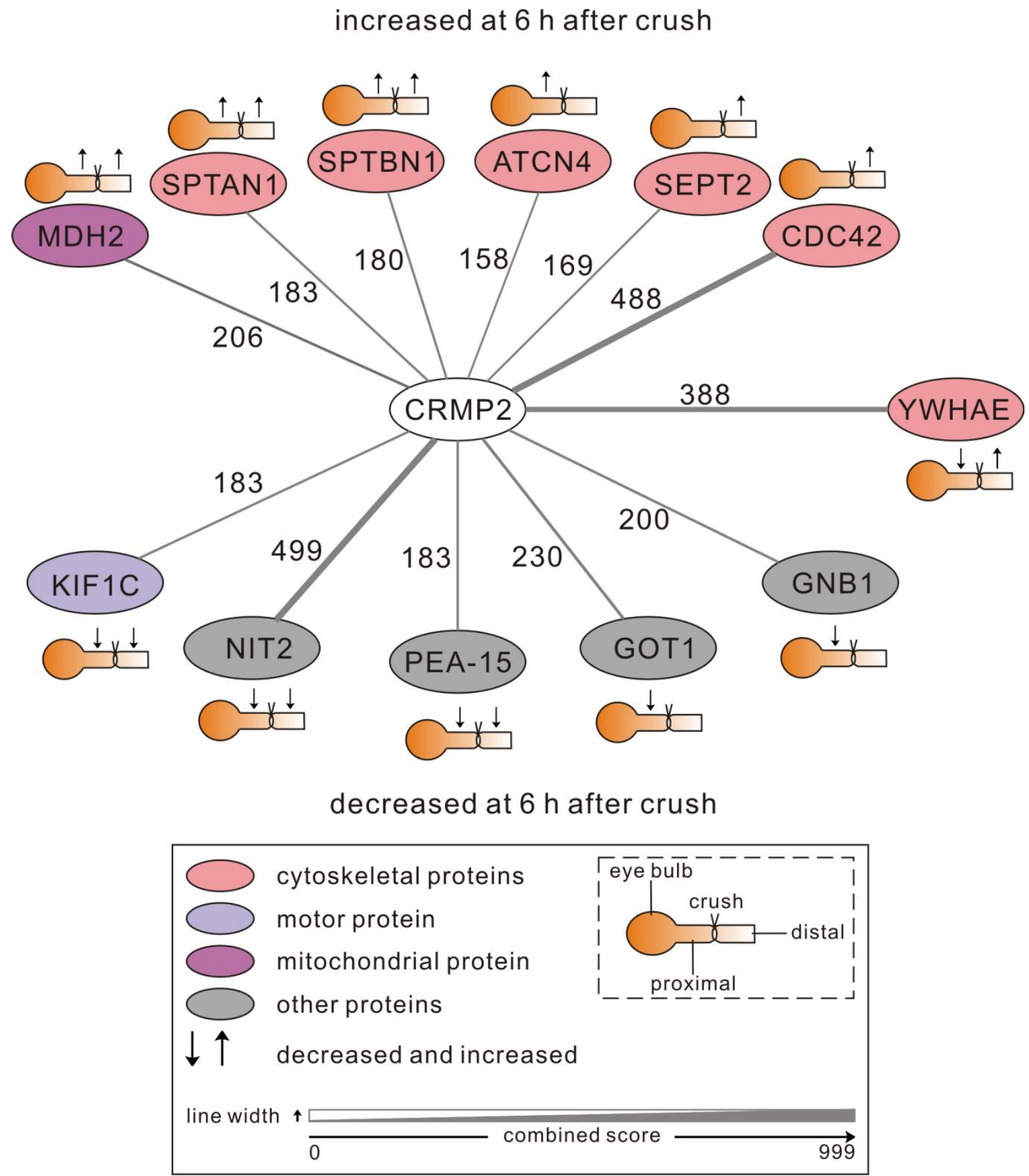

Figure 3.18 CRMP2-interacting partners with altered expression levels during acute axonal degeneration.

Optic nerve lysates were prepared from two areas comprising $1 \mathrm{~mm}$ proximal or distal from the lesion at $6 \mathrm{~h}$ after crush. Out of 2685 identified proteins, the expression levels of 135 proteins were significantly regulated between groups (assessed by ANOVA analysis). 12 proteins of these 135 regulated proteins were 
identified as CRMP2-interacting partners by a STRING (v10 for rat) database search. These proteins are shown in this figure. When the fold change relative to control is more than 1.3 or less than -1.3 , the protein levels are considered increased $(\boldsymbol{T})$ or decreased $(\boldsymbol{})$ in proximal and/or distal part during AAD. These 12 proteins are classified according to their functions represented in different colors. The edge width of the connecting lines and the adjacent numbers represent the combined scores from the STRING database. The combined score correlates to the level of evidence of a protein-protein interaction.

Abbreviations for the 12 regulated CRMP2-interacting partners: ACTN4 = alpha-actinin 4; CDC42 = cell division control protein 42; GNB1 = G protein beta subunit $1 ;$ GOT1 = aspartate transaminase 1 ; $\mathrm{KIF}=$ kinesin-like protein; $\mathrm{MDH} 2$ = malate dehydrogenase 2; NIT2 = omega-amidase; $\mathrm{PEA}-15$ = astrocytic phosphoprotein 15; SEPT2 = septin 2; SPTAN1 = alpha-II spectrin; SPTBN1 = beta-II spectrin; YWHAE = 14-3-3 protein epsilon. 


\section{Discussion}

Axonal degeneration plays a central pathophysiological role in many neurodegenerative, neuroinflammatory and neurotraumatic diseases. Therefore it is important to better understand its underlying molecular mechanisms. Traumatic lesion models are used to study the mechanisms of traumatic axonal degeneration, which can, at least partly, be transferred to other forms of axonal degeneration (Raff et al., 2002; Wang et al., 2012). AAD is of special interest not only because it resembles other relevant forms of axonal degeneration like FAD, but also because it affects the proximal part of the axon, which is the basis for later axonal regeneration. Prior to the present study, calcium influx was shown to be the crucial initiating event in AAD (Knöferle et al., 2010). Moreover, a rapid calpain activation had been demonstrated in AAD of the mouse spinal cord and in some in vitro models (Kerschensteiner et al., 2005). However, the further molecular mechanisms of AAD following calcium influx were still unknown and have thus been studied here.

In the present work, the crucial role of calpain in AAD was demonstrated in the optic nerve and characterized with regards to the time kinetics and localization of its activation. CRMP2 was newly identified as the most important downstream target of calpain in AAD. Effects of CRMP2 on axonal mitochondria transport were examined in vitro suggesting that they are central to CRMP2 function in AAD. Finally, molecular interactors of CRMP2 in AAD were identified using proteomics analysis of the optic nerve.

\subsection{The role of calpain in acute axonal degeneration of the optic nerve in vivo}

$A A D$ is initiated by a rapid transient calcium influx into the axon induced by the lesion (Knöferle et al., 2010). One of the putative molecular downstream targets is the calcium dependent protease calpain (Kampfl et al., 1997). Calpain activation has been previously reported to play an important role in many neurodegenerative disease models (Vosler, 2009). Several studies have shown that calpain is activated early during traumatic axonal degeneration using different in vitro and in vivo models (George et al., 1995; Huh et al., 2006; 
Kilinc et al., 2009; Saatman et al., 2003; Spira et al., 2003). For example, calpain was activated at 30 min after spinal cord injury (Kerschensteiner et al., 2005) as well as in all cortex layers of 11- and 17-day old rats at $6 \mathrm{~h}$ after traumatic brain injury (Huh et al., 2006). In cultured chick forebrain neurons in vitro, calpain activation was observed in the first minutes after shear stress (Kilinc et al., 2009). The kinetics of calpain activation varies in different models. Here, we investigated the role and kinetics of calpain activation during AAD of the optic nerve in vivo following ONC.

\subsubsection{Calpain is activated early during acute axonal degeneration}

To investigate the role of calpain in AAD of the optic nerve, we evaluated the expression of the $145 \mathrm{kDa}$ spectrin-BDP before and at various time points after ONC. We found that calpain activation is detected as early as $5 \mathrm{~min}$ after ONC. A continuous increase of calpain activity was observed on both sides of the crush over the following $6 \mathrm{~h}$. Calpain activation was initially faster on the proximal side as compared to the distal side, but reached similar levels at $6 \mathrm{~h}$ after ONC.

In the rat ONC model, structural changes of the cytoskeleton have been observed already at $30 \mathrm{~min}$ after lesion (Knöferle et al., 2010). Moreover, calpain-mediated neurofilament proteolysis has been reported at later time-points after transection of the optic nerve in mice (Ma et al., 2013). We demonstrate here a significant cleavage of the cytoskeletal protein spectrin already at $5 \mathrm{~min}$ after ONC. Thus, early calpain activation plays an important role in the rapid and prolonged cytoskeleton breakdown during $A A D$ of the optic nerve.

We performed immunohistochemical analysis of optic nerve sections to specify the location of calpain activation. The employed antibody specifically recognizes the calpain-derived spectrin-BDP (Roberts-Lewis et al., 1994). We found that intra-axonal spectrin-BDP staining intensity was significantly increased in both proximal and distal parts at $1 \mathrm{~h}$ after ONC, showing that calpain was activated specifically in the axons of the optic nerve. Interestingly, we found that the increased staining signal of spectrin-BDP was limited to the area affected by $A A D$. In line with this finding, a previous study showed that the increased levels of the calpain-derived spectrin-BDP were observed in a similar area adjacent 
to the lesion side at 30 min after spinal cord injury (Kerschensteiner et al., 2005). Another recent study from our group showed that the levels of the autophagy proteins ATG5 and ULK1 were increased specifically in the same area near the lesion site after spinal cord injury (Ribas et al., 2015). Therefore the spread of calpain activation most probably defines the spatial boundaries of AAD along the axon and is reflected by the later spatial distribution of other downstream molecules in AAD followed by axonal fragmentation.

\subsubsection{Calpain inhibition attenuates crush-induced acute axonal degeneration in vivo}

The neuroprotective effect of calpain inhibition by pharmacological compounds or genetic techniques has been shown in several models of neurodegeneration (Araújo et al., 2004; Das et al., 2014; Hanlon et al., 2003; Ma et al., 2012; Samantaray et al., 2015; Yang et al., 2013). For example, it was shown before that a calpain inhibitor was neuroprotective during WD in

the opossum optic nerve (Araújo et al., 2004). Calpain inhibition was also reported to attenuate demyelination and axonal damage in the EAE mouse model (Das et al., 2014). The effects of calpain inhibition on AAD in the mammalian optic nerve have, however, not been studied before.

In order to determine whether calpain inhibition interferes with AAD in vivo, we evaluated the effects of the cell-penetrating calpain inhibitor calpeptin (Mehdi, 1991) on AAD by in-vivo live imaging of the optic nerve. Intravitreal injection of calpeptin at $2.5 \mathrm{~h}$ before ONC effectively inhibited calpain activation during the first $6 \mathrm{~h}$ after ONC. Importantly, calpeptin treatment completely inhibited axonal fragmentation up to $6 \mathrm{~h}$ after $\mathrm{ONC}$ in the proximal part of the axon. The protective effect was less pronounced in the distal part of the axon. Our results are in line with a previous study of $A A D$ in the spinal cord, which showed that calpain inhibition attenuated axonal fragmentation at 1 hour after injury (Kerschensteiner et al., 2005). We show for the first time that the axon protective effects of calpain inhibition in AAD persist over $6 \mathrm{~h}$ after lesion. These findings demonstrate that calpain activation is a major molecular step in AAD following calcium influx. 


\subsubsection{CRMP2 is an important downstream target of calpain during acute axonal degeneration}

Until now it is not known which molecular mechanisms follow downstream of calcium influx and calpain activation in AAD. To identify further downstream targets of the protease calpain, we checked whether calpain targets that had been previously described in other models were also cleaved during AAD in the optic nerve. We focused on proteins that are involved in central mechanisms of axonal degeneration including autophagy, cytoskeleton integrity and axonal transport (Atalay et al., 2007; Coleman, 2005; Knöferle et al., 2010; Liu et al., 2011; Russo et al., 2011; Yoon et al., 2008; Yousefi et al., 2006; Zhang et al., 2007). Besides a cleavage of the microtubule-associated protein MAP2 on the proximal side of the crush only, we found a strong and specific cleavage of CRMP2 in both proximal and distal axon parts during AAD. To investigate whether calpain activation is indeed responsible for the cleavage of CRMP2, we evaluated the effect of calpain inhibition on the levels of cleaved CRMP2 during AAD of the optic nerve. We could show that the calpain inhibitor calpeptin significantly suppresses the cleavage of CRMP2 until $6 \mathrm{~h}$ after ONC. This was confirmed in a scratch lesion model in primary cortical neurons in vitro.

The protein CRMP2 is known to regulate axonal outgrowth by linking axonal transport to the cytoskeleton (Fukata et al., 2002; Kawano et al., 2005; Kimura et al., 2005). Altered expression of CRMP2 has been described in a few other neurodegenerative disease models before (Khanna, 2013). In primary cortical neurons, it was shown before that intact CRMP2 was decreased after neurotoxic injury (Zhang et al., 2007). Another study showed that phosphorylated CRMP2 was increased in the regenerating spinal cord in chicks after injury (Gögel et al., 2010). Our current data provides the first evidence that calpain-mediated cleavage of CRMP2 plays a central role in AAD. 


\subsection{The role of CRMP2 in acute axonal degeneration in vitro and in vivo}

Based on these findings, we speculated that overexpression of intact CRMP2 might protect against axonal degeneration. Therefore, we investigated the role of CRMP2 overexpression in $A A D$ in vitro and in vivo.

\subsubsection{CRMP2 overexpression delays acute axonal degeneration}

To examine the effects of CRMP2 overexpression on AAD in vitro, we imaged axonal changes of primary cortical neurons in the microfluidic chamber system after axotomy. Interestingly, up to $8 \mathrm{~h}$ after axotomy in control neurons, we did not detect any axonal fragmentation, which is different from the findings in vivo. However, an increasing number of axonal bulbs was formed which could be correlated to axonal degeneration. The differences of axonal degeneration in vitro and in vivo can be explained by the different developmental stage of the lesioned neurons (embryonal, outgrowing axons in vitro versus adult, synaptically connected axons in vivo) and the differences in the cellular environment (pure neuronal in vitro versus glial cells and other tissues in vivo). The fact that the embryonal axons in vitro are more resistant against lesion is an interesting finding that should be followed up in the future with regards to the putatively different molecular prerequisites.

We found that CRMP2 overexpression significantly delays the formation of axonal bulbs up to $8 \mathrm{~h}$ after axotomy. Since the formation of axonal bulbs is considered an early hallmark of axonal degeneration prior to axonal fragmentation (Coleman, 2005; Lingor et al., 2012; William et al., 1997), our in-vitro data suggest that CRMP2 overexpression inhibits the process of axonal degeneration. It had been shown previously that neurite swellings of lesioned mouse superior cervical ganglia cells contain increased levels of cleaved CRMP2 (Touma et al., 2007). However, this study did not compare levels of intact CRMP2 along the axon nor did it perform any functional testing with regards to CRMP2 levels. Our results show that increasing the levels of intact CRMP2 is sufficient to inhibit bulb formation and to overcome putative local detrimental effects of the CRMP2 cleavage product in vitro.

We further evaluated the effects of CRMP2 overexpression on AAD by live imaging of the optic nerve in vivo. We found that viral vector-mediated CRMP2 overexpression effectively 
protected the proximal part of the axon against axonal fragmentation during AAD of the optic nerve. Also on the distal side, $A A D$ was attenuated by trend in the axons overexpressing CRMP2. The degree of the attenuating effect of CRMP2 overexpression on AAD in the optic nerve was comparable to the effect of calpain inhibition, which suggests that CRMP2 is a central downstream target of calpain during $A A D$ and which further elucidates the molecular pathway of AAD. Besides, we show here for the first time that increasing CRMP2 levels effectively counteracts axonal degeneration in vivo.

There are divergent data regarding the biological effects of the calpain derived cleavage product of CRMP2. In primary mouse cortical neurons it was shown that cleaved CRMP2 increased the resistance against excitotoxic insults through modulating the expression of NMDA receptors and synaptic plasticity (Bretin et al., 2006). However, another study in cortical neurons in vitro and in a traumatic brain injury model in vivo showed that the cleavage of CRMP2 contributes to neuronal cell death and inhibits neuronal regeneration (Zhang et al., 2007). These divergent results suggest differential effects of the CRMP2 cleavage product depending on the time-scale of its action. Moreover, it is still not understood whether the effects of CRMP2 on neurodegenerative disorders are mediated by a loss-of-function of the intact CRMP2 (which is being cleaved) or a gain-of-function of the cleavage product. In our study, overexpression of intact CRMP2 markedly interfered with axonal degeneration, while no changes in the level of cleaved CRMP2 could be detected. These results argue for an important function of the intact CRMP2 for axonal integrity during $A A D$ rather than a specific toxic effect of the CRMP2 cleavage product.

\subsubsection{CRMP2 overexpression rescues the impairment of mitochondrial transport during} acute axonal degeneration

Our group previously reported that the cytoskeleton is affected early in AAD and that axonal bulbs consist of a local accumulation of organelles (Knöferle et al., 2010). These results suggest an impairment of axonal transport as one of the earliest and important events during AAD. Therefore, we examined the kinetics of mitochondrial transport after axotomy in the microfluidic chamber system. We found that the percentage of motile 
mitochondria was strongly decreased within the first hour after axotomy. Interestingly, all analyzed transport parameters recovered to normal levels at $2 \mathrm{~h}$ after lesion. These results demonstrate for the first time an early but transient impairment of mitochondrial transport after axotomy. The time-course correlates with the beginning of bulb formation and organelle accumulation as seen in the cortical neurons in vitro and as reported before in vivo (Knöferle et al., 2010). Thus, impairment of axonal transport is most probably one of the important causal factors for the early morphological changes seen in AAD. This notion is further supported by our findings showing that CRMP2 overexpression can rescue both AAD and axonal transport, as discussed below. Future experiments are necessary to examine whether the kinetics of mitochondrial transport are similar in vivo. Especially the recovery of axonal transport at $2 \mathrm{~h}$ after lesion might be specific for the in vitro condition where no axonal fragmentation occurs and where axons have been shown to successfully regenerate within $72 \mathrm{~h}$ after lesion, which is not the case in vivo.

In our current study, we have demonstrated that CRMP2 overexpression delays bulb formation after axotomy and attenuates AAD in vivo. However, the underlying mechanisms still need to be studied. It has been reported that CRMP2 interacts with the motor proteins kinesin and dynein (Arimura, Hattori, et al., 2009; Kimura et al., 2005). Thus, we investigated the effect of CRMP2 overexpression on the kinetics of mitochondrial transport after axotomy in vitro. We found that overexpression of CRMP2 did not affect the percentage or speed of mitochondrial transport in unlesioned axons. However, CRMP2 overexpression completely rescued the impaired mitochondrial transport at 30 min after axotomy. Interestingly, the most pronounced attenuating effect of CRMP2 overexpression on axonal bulb formation coincided at the same time point, 30 min after axotomy. At later time points, continuous bulb formation was observed also in the CRMP2 overexpressing axons in vitro. These results suggest that overexpression of CRMP2 inhibits bulb formation mainly by counteracting the early transient impairment of axonal transport after axotomy. Moreover, there seem to be different time-dependent stages of bulb formation in AAD with putatively different molecular mechanisms. If this was true, then axonal transport impairment would result in the formation of bulbs only at early time-points in AAD. The mechanisms underlying the later 
bulb formation in AAD still need to be explored. Mechanistically, the impairment of axonal transport could be caused by a decreased binding of cleaved CRMP2 to motor proteins, which is one of the most important functions of intact CRMP2 (Arimura, Hattori, et al., 2009; Kawano et al., 2005; Kimura et al., 2005).

\subsection{Proteomics analysis of acute axonal degeneration in the optic nerve}

Proteomics analysis of axonal degeneration in the CNS has so far only been performed at later time points, for example at $24 \mathrm{~h}$ and $48 \mathrm{~h}$ after ONC, where the expression levels of 27 actin cytoskeleton associated proteins were significantly altered (Garland et al., 2012). Here, we performed proteomics analysis at $6 \mathrm{~h}$ after ONC compared to control group to characterize further molecular targets that contribute to AAD. Out of the differentially expressed proteins during $A A D$, we identified 12 CRMP2-interacting proteins.

Notably, the motor protein KIF1C was decreased on both proximal and distal sides of the crush. KIF1 is a member of the kinesin-3 family, which regulates axonal transport along microtubules (Hirokawa et al., 2009). It was previously reported that, after CRMP2 directly binds to KIF and forms a complex to regulate tubulin transport (Kimura et al., 2005). Thus, the reduced level of KIF1C might augment the negative effect of CRMP2 cleavage on axonal transport.

Several cytoskeleton associated proteins that interact with CRMP2 were increased during AAD, including SPTAN1 and SPTBN1, CDC42, SEPT2 and ACTN4. The proteins SPTAN1 and SPTBN1 are different subunits of spectrin that play an important role to maintain membrane stability and link motor proteins to filament systems (De Matteis and Morrow, 2000). Cdc42, a member of the Rho GTPase family, regulates the growth and stability of microtubules and actin structures (Etienne-Manneville, 2004). SEPT2 belongs to a family of GTP-binding proteins, and it has been reported to regulate the actin and microtubule cytoskeleton (Spiliotis, 2010). ACTN4 has also been reported to regulate the actin cytoskeleton (Otey and Carpen, 2004). The accumulation of these cytoskeleton proteins might be induced by the impairment of axonal transport during $A A D$ as they are usually transported continuously 
along the axon. Such a rapid accumulation of synaptic vesicle proteins after blocking axonal transport was reported before for synaptophysin and alpha-synuclein in the optic nerve after crush (Li and Dahlström, 1997).

The increased levels of the mitochondrial protein $\mathrm{MDH} 2$ on both sides of the crush most probably reflect the accumulation of mitochondria during $A A D$ due to impaired mitochondrial transport.

PEA-15 was decreased on both sides of the crush. PEA-15 can activate the ERK/MAPK signaling pathway, which regulates neuronal cell survival (Formstecher et al., 2001; Ramos et al., 2000). Thus, PEA-15 is a promising molecular target to biologically confirm and to follow up on in the future.

The levels of GOT1, NIT2 and GNB1 were decreased during AAD. These proteins are involved in glutamate metabolism and signal transduction (Erecińska and Silver, 1990). How PEA-15, GOT1, NIT2 and GNB1 contribute to AAD still needs to be further investigated.

Taken together, the proteomics analysis further confirmed that CRMP2 is an important molecular mediator in AAD, as several interactors of CRMP2 that belong to relevant molecular pathways in axonal degeneration were found to be regulated. Moreover, a number of molecules was identified that need to be further evaluated in the future and that possibly represent key steps in the molecular cascade of axonal degeneration and promising therapeutic targets in AAD.

\subsection{Conclusion}

In conclusion, this study provides novel knowledge about the molecular mechanisms involved in AAD. A continuous increase in calpain activity is demonstrated following calcium influx. Calpain then cleaves CRMP2, which impairs mitochondrial transport. Both calpain inhibition and CRMP2 overexpression almost completely inhibit axonal fragmentation proximal to the lesion site during AAD. Finally, several CRMP2-interacting partners are regulated during $A A D$ and might contribute to $A A D$. Their relevance needs to be examined in further studies. Thus, this study provides new insights into the molecular mechanisms of 
axonal degeneration, and identifies novel therapeutic targets in traumatic and degenerative diseases of the CNS (Figure 4.1).

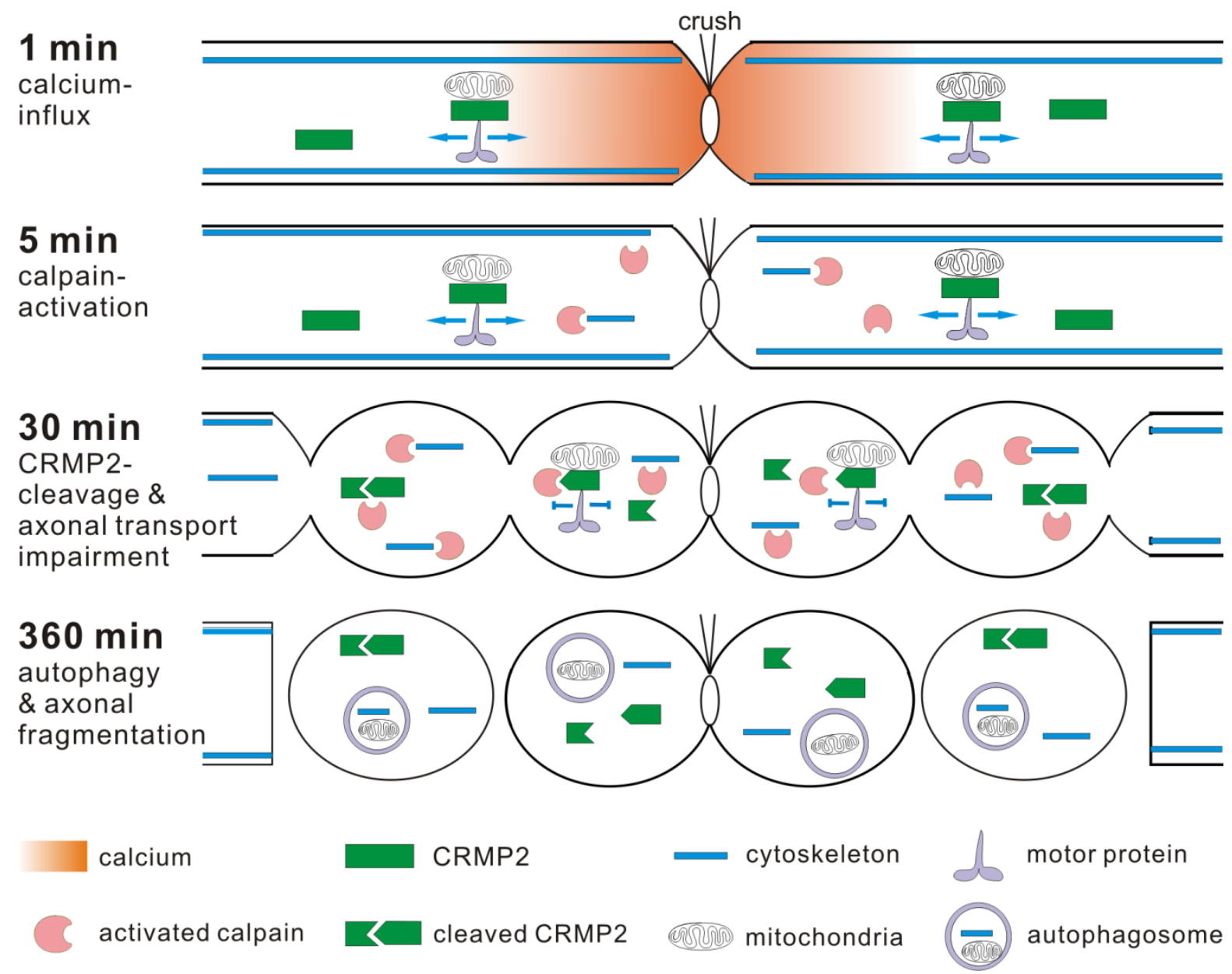

Figure 4.1 Schematic drawing of the molecular mechanisms of acute axonal degeneration.

Influx of extracellular calcium activates calpain, which in turn leads to the cleavage of CRMP2. The resulting disruption of the binding between CRMP2 and motor proteins is most probably responsible for the impairment of axonal transport. Besides, calpain results in the degradation of the cytoskeleton. Autophagy is also induced by calcium influx and degrades cellular materials and organelles. 


\section{Summary}

The development of neuroprotective treatments is urgently needed for neurodegenerative and neurotraumatic diseases. As axonal degeneration is a key initiating event in these diseases, it is pivotal to better understand and counteract axonal degeneration. A traumatic lesion of axons in the CNS is followed by AAD within several hours. However, the underlying molecular mechanisms of AAD are still incompletely understood.

In this study, we investigated the mechanisms of AAD following calcium influx after ONC in vivo and after axotomy of cortical neurons in the microfluidic chamber system in vitro. We found that calpain, a calcium-dependent protease, was activated within the first minutes during $A A D$ of the optic nerve. Calpain activation was localized specifically in the axons, and limited to the area affected by AAD. Calpain inhibition almost completely inhibited axonal fragmentation on the proximal side over $6 \mathrm{~h}$ after crush while its protective effect on the distal side was less pronounced. Furthermore, we demonstrated that CRMP2 is the main downstream target of calpain activation during AAD. Overexpression of CRMP2 significantly delayed formation of axonal bulbs proximal to the lesion site over $8 \mathrm{~h}$ after axotomy in vitro, which suggests that CRMP2 represents a crucial step in AAD. This was confirmed during AAD of the optic nerve in vivo, where AAV-mediated CRMP2-overexpression completely inhibited axonal fragmentation in the proximal part. Considering the interactions between CRMP2 and motor proteins, we examined the effect of CRMP2 on mitochondrial transport after axotomy. The results showed that mitochondrial transport was impaired early after axotomy while CRMP2 overexpression rescued this impairment. Finally, the regulation of several CRMP2-interacting partners was identified during AAD of the optic nerve by proteomics analysis. These proteins included cytoskeletal proteins, mitochondrial proteins, as well as the proteins which are involved in axonal transport, ERK/MAPK signaling and glutamate metabolism.

Taken together, this study adds new knowledge about the mechanisms of AAD. Moreover, it identifies new therapeutic targets in traumatic and degenerative diseases of the CNS, of which CRMP2 seems to be one of the most promising ones. 


\section{References}

Adalbert R, Morreale G, Paizs M, Conforti L, Walker SA, Roderick HL, et al. Intra-axonal calcium changes after axotomy in wild-type and slow Wallerian degeneration axons. Neuroscience 2012; 225: 44-54.

Adalbert R, Nógrádi A, Szabó A, Coleman MP. The slow Wallerian degeneration gene in vivo protects motor axons but not their cell bodies after avulsion and neonatal axotomy. Eur. J. Neurosci. 2006; 24: 2163-2168.

Alavi MV, Bette S, Schimpf S, Schuettauf F, Schraermeyer U, Wehrl HF, et al. A splice site mutation in the murine Opa1 gene features pathology of autosomal dominant optic atrophy. Brain 2007; 130: 1029-1042.

Araki T, Sasaki Y, Milbrandt J. Increased nuclear NAD biosynthesis and SIRT1 activation prevent axonal degeneration. Science 2004; 305: 1010-1013.

Araújo Couto L, Narciso MS, Hokoç JN, Martinez AMB. Calpain inhibitor 2 prevents axonal degeneration of opossum optic nerve fibers. J. Neurosci. Res. 2004; 77: 410-419.

Arduíno DM, Esteves AR, Cardoso SM. Mitochondria drive autophagy pathology via microtubule disassembly: A new hypothesis for Parkinson disease. Autophagy 2013; 9: 112-114.

Arimura N, Hattori A, Kimura T, Nakamuta S, Funahashi Y, Hirotsune S, et al. CRMP-2 directly binds to cytoplasmic dynein and interferes with its activity. J. Neurochem. 2009; 111: 380-390.

Arimura N, Kawano Y, Yoshimura T, Kawabata S, Hattori A, Fukata Y, et al. Phosphorylation by Rho Kinase Regulates CRMP-2 Activity in Growth Cones. Mol. Cell. Biol. 2005; 25: 9973-9984.

Arimura N, Kimura T, Nakamuta S, Taya S, Funahashi Y, Hattori A, et al. Anterograde Transport of TrkB in Axons Is Mediated by Direct Interaction with SIp1 and Rab27. Dev. Cell. 
2009; 16: 675-686.

Ashburner M, Ball CA, Blake JA, Botstein D, Butler H, Cherry JM, et al. Gene ontology: tool for the unification of biology. The Gene Ontology Consortium. Nat. Genet. 2000; 25: 25-29.

Atalay B, Caner H, Can A, Cekinmez M. Attenuation of microtubule associated protein-2 degradation after mild head injury by mexiletine and calpain-2 inhibitor. Br. J. Neurosurg. 2007; 21: 281-287.

Atanassov I, Urlaub $\mathrm{H}$. Increased proteome coverage by combining PAGE and peptide isoelectric focusing: Comparative study of gel-based separation approaches. Proteomics 2013; 13: 2947-2955.

Avery MA, Rooney TM, Pandya JD, Wishart TM, Gillingwater TH, Geddes JW, et al. WIdS prevents axon degeneration through increased mitochondrial flux and enhanced mitochondrial $\mathrm{Ca}^{2+}$ buffering. Curr. Biol. 2012; 22: 596-600.

Bähr M. Live or let die - retinal ganglion cell death and survival during development and in the lesioned adult CNS. Trends Neurosci. 2000; 23:483-490.

Beirowski B, Babetto E, Gilley J, Mazzola F, Conforti L, Janeckova L, et al. Non-nuclear WId(S) determines its neuroprotective efficacy for axons and synapses in vivo. J. Neurosci. 2009; 29: 653-668.

Bilsland LG, Sahai E, Kelly G, Golding M, Greensmith L, Schiavo G. Deficits in axonal transport precede ALS symptoms in vivo. Proc. Natl. Acad. Sci. U. S. A. 2010; 107: 20523-20528.

Bretin S, Marin P, Maus M, Torrens $\mathrm{Y}$, Glowinski J, Lyon CB, et al. Calpain product of WT-CRMP2 reduces the amount of surface NR2B NMDA receptor subunit. J. Neurochem. 2006; 98(4): 1252-1265.

Burke RE, O'Malley K. Axon Degeneration in Parkinson's Disease. Exp. Neurol. 2013; 246: 72-83. .

Cai X, Yuan R, Hu Z, Chen C, Yu J, Zheng Z, et al. Expression of PirB Protein in Intact and Injured Optic Nerve and Retina of Mice. Neurochem. Res. 2012; 37: 647-654. 
Cavanagh JB. The significance of the 'dying back' process in experimental and human neurological disease. Int. Rev. Exp. Pathol. 1964; 3: 219-67.

Cheng H-C, Kim SR, Oo TF, Kareva T, Yarygina O, Rzhetskaya M, et al. Akt suppresses retrograde degeneration of dopaminergic axons by inhibition of macroautophagy. J. Neurosci. 2011; 31: 2125-2135.

Cho K, Yang L, Lu B, Ma HF, Huang X, Pekny M, et al. Re-establishing the regenerative potential of central nervous system axons in postnatal mice. J. Cell. Sci. 2005; 118: 863-872.

Coleman M. Axon degeneration mechanisms: commonality amid diversity. Nat. Rev. Neurosci. 2005; 6: 889-898.

Conforti L, Gilley J, Coleman MP. Wallerian degeneration: an emerging axon death pathway linking injury and disease. Nat. Rev. Neurosci. 2014; 15: 394-409

Craner MJ, Fugger L. Axonal injury in reverse. Nat. Med. 2011; 17: 423-426.

Cui B, Wu C, Chen L, Ramirez A, Bearer EL, Li W-P, et al. One at a time, live tracking of NGF axonal transport using quantum dots. Proc. Natl. Acad. Sci. U. S. A. 2007; 104: 13666-13671.

Das A, Guyton MK, Smith A, Iv GW, Mcdowell ML, Matzelle DD, et al. Calpain inhibitor attenuated optic nerve damage in acute optic neuritis in rats. J. Neurochem. 2014; 124: 133-146.

De vos KJ, Chapman AL, Tennant ME, Manser C, Tudor EL, Lau KF, et al. Familial amyotrophic lateral sclerosis-linked SOD1 mutants perturb fast axonal transport to reduce axonal mitochondria content. Hum. Mol. Genet. 2007; 16: 2720-2728.

Dotti CG, Sullivan CA, Banker GA. The establishment of polarity by hippocampal neurons in culture. J. Neurosci. 1988; 8(4): 1454-68.

Erecińska M, Silver IA. Metabolism and role of glutamate in mammalian brain. Prog. Neurobiol. 1990; 35: 245-296.

Etienne-Manneville S. Actin and microtubules in cell motility: Which one is in control? Traffic 
2004; 5: 470-477.

Formstecher E, Ramos JW, Fauquet M, Calderwood D A, Hsieh JC, Canton B, et al. PEA-15 Mediates Cytoplasmic Sequestration of ERK MAP Kinase. Dev. Cell 2001; 1: 239-250.

Freeman MR. Signaling mechanisms regulating Wallerian degeneration. Curr Opin Neurobiol 2014; 27C: 224-231.

Fukata $\mathrm{Y}$, Itoh TJ, Kimura T, Ménager C, Nishimura T, Shiromizu T, et al. CRMP-2 binds to tubulin heterodimers to promote microtubule assembly. Nat. Cell Biol. 2002; 4: 583-591.

Garland P, Broom L, Quraishe S, Dalton PD, Skipp P, Newman TA, et al. Soluble Axoplasm Enriched from Injured CNS Axons Reveals the Early Modulation of the Actin Cytoskeleton. PLoS One 2012; 7: 1-8.

Gennarelli TA, Thibault LE, Tipperman R, Tomei G, Sergot R, Brown M, et al. Axonal injury in the optic nerve: a model simulating diffuse axonal injury in the brain. J. Neurosurg. 1989; 71: 244-253.

George EB, Glass JD, Griffin JW. Axotomy-induced axonal degeneration is mediated by calcium influx through ion-specific channels. J. Neurosci. 1995; 15: 6445-6452.

Ghaffarieh A, Levin LA. Optic Nerve Disease and Axon Pathophysiology. Int. Rev. Neurobiol. 2012; 105: 1-17.

Gilley J, Coleman MP. Endogenous Nmnat2 Is an Essential Survival Factor for Maintenance of Healthy Axons. PLoS. Biol. 2010; 8(1):e1000300

Gögel S, Lange S, Leung KY, Greene NDE, Ferretti P. Post-translational regulation of Crmp in developing and regenerating chick spinal cord. Dev. Neurobiol. 2010; 70: 456-471.

Hanlon GMO, Humphreys PD, Goldman RS, Halstead SK, Bullens RWM, Plomp JJ, et al. Calpain inhibitors protect against axonal degeneration in a model of anti-ganglioside antibody-mediated motor nerve terminal injury. Brain 2003; 126(Pt 11): 2497-509.

Hensley K, Venkova K, Christov A, Gunning W, Park J. Collapsin response mediator protein-2: 
An emerging pathologic feature and therapeutic target for neurodisease indications. Mol. Neurobiol. 2011; 43: 180-191.

Hirokawa N, Noda Y, Tanaka Y, Niwa S. Kinesin superfamily motor proteins and intracellular transport. Nat. Rev. Mol. Cell Biol. 2009; 10: 682-696.

Hoopfer ED, McLaughlin T, Watts RJ, Schuldiner O, O'Leary DDM, Luo L. Wlds Protection Distinguishes Axon Degeneration following Injury from Naturally Occurring Developmental Pruning. Neuron 2006; 50: 883-895.

Hosie KA, King AE, Blizzard CA, Vickers JC, Dickson TC. Chronic excitotoxin-induced axon degeneration in a compartmented neuronal culture model. ASN. Neuro. 2012; 4: 47-57.

Howell GR, Libby RT, Jakobs TC, Smith RS, Phalan FC, Barter JW, et al. Axons of retinal ganglion cells are insulted in the optic nerve early in DBA/2J glaucoma. J. Cell Biol. 2007; 179: 1523-1537.

Huh JW, Franklin MA, Widing AG, Raghupathi R. Regionally distinct patterns of calpain activation and traumatic axonal injury following contusive brain injury in immature rats. Dev. Neurosci. 2006; 28: 466-476.

Ivins KJ, Bui ET, Cotman CW. Beta-amyloid induces local neurite degeneration in cultured hippocampal neurons: evidence for neuritic apoptosis. Neurobiol. Dis. 1998; 5: 365-378

Jawhar S, Trawicka A, Jenneckens C, Bayer TA, Wirths O. Motor deficits, neuron loss, and reduced anxiety coinciding with axonal degeneration and intraneuronal $A \beta$ aggregation in the 5XFAD mouse model of Alzheimer's disease [Internet]. Neurobiol. Aging 2012; 33: 196.e29-196.e40.

Johnson GV, Litersky JM, Jope RS. Degradation of microtubule-associated protein 2 and brain spectrin by calpain: a comparative study. J. Neurochem. 1991; 56: 1630-1638.

Kampfl A, Posmantur RM, Zhao X, Schmutzhard E, Clifton GL, Hayes RL. Mechanisms of calpain proteolysis following traumatic brain injury: implications for pathology and therapy: implications for pathology and therapy: a review and update. J. Neurotrauma 1997; 14: 
121-134.

Kanamori A, Catrinescu MM, Belisle JM, Costantino S, Levin LA. Retrograde and Wallerian axonal degeneration occur synchronously after retinal ganglion cell axotomy. Am. J. Pathol. 2012; 181: 62-73.

Kawaguchi I, Higashide T, Ohkubo S, Takeda H, Sugiyama K. In Vivo Imaging and Quantitative Evaluation of the Rat Retinal Nerve Fiber Layer Using Scanning Laser Ophthalmoscopy. Investig. Opthalmology Vis. Sci. 2006; 47: 2911.

Kawano Y, Yoshimura T, Tsuboi D, Kaneko-kawano T, Shirataki H, Kawabata S et al. CRMP-2 Is Involved in Kinesin-1-Dependent Transport of the Sra-1 / WAVE1 Complex and Axon Formation CRMP-2 Is Involved in Kinesin-1-Dependent Transport of the Sra-1 / WAVE1 Complex and Axon Formation . Mol. Cell. Biol. 2005; 25(22):9920-35.

Kerschensteiner M, Schwab ME, Lichtman JW, Misgeld T. In vivo imaging of axonal degeneration and regeneration in the injured spinal cord. Nat. Med. 2005; 11: 572-577.

Khanna R. Opening Pandora's jar: a primer on the putative roles of CRMP2 in a panoply of neurodegenerative, sensory and motor neuron, and central disorders. Future. Neurol. 2013; 7: 749-771.

Kilinc D, Gallo G, Barbee KA. Mechanical membrane injury induces axonal beading through localized activation of calpain. Exp. Neurol. 2009; 219: 553-561.

Kimura T, Arimura N, Fukata Y, Watanabe H, Iwamatsu A, Kaibuchi K. Tubulin and CRMP-2 complex is transported via Kinesin-1. J. Neurochem. 2005; 93: 1371-1382.

Knöferle J, Koch JC, Ostendorf T, Michel U, Planchamp V, Vutova P, et al. Mechanisms of acute axonal degeneration in the optic nerve in vivo. Proc. Natl. Acad. Sci. U. S. A. 2010; 107: 6064-6069.

Koch JC, Barski E, Lingor P, Bähr M, Michel U. Plasmids containing NRSE/RE1 sites enhance neurite outgrowth of retinal ganglion cells via sequestration of REST independent of NRSE dsRNA expression. FEBS J. 2011; 278: 3472-3483. 
Koch JC, Bitow F, Haack J, d'Hedouville Z, Zhang J-N, Tönges L, et al. Alpha-Synuclein affects neurite morphology, autophagy, vesicle transport and axonal degeneration in CNS neurons. Cell Death Dis. 2015; 6: e1811.

Koch JC, Knöferle J, Tönges L, Michel U, Bähr M, Lingor P. Imaging of rat optic nerve axons in vivo. Nat. Protoc. 2011; 6: 1887-96.

Koch JC, Solis GP, Bodrikov V, Michel U, Haralampieva D, Shypitsyna A, et al. Upregulation of reggie-1/flotillin-2 promotes axon regeneration in the rat optic nerve in vivo and neurite growth in vitro. Neurobiol. Dis. 2013; 51: 168-176.

Koch JC, Tönges L, Barski E, Michel U, Bähr M, Lingor P. ROCK2 is a major regulator of axonal degeneration, neuronal death and axonal regeneration in the CNS. Cell Death Dis. 2014; 5: e1225.

Lee JN, Jiang X, Ryan D, Whitesides GM. Compatibility of mammalian cells on surfaces of poly(dimethylsiloxane). Langmuir 2004; 20: 11684-11691.

Lenaers G, Hamel CP, Delettre C, Amati-Bonneau P, Procaccio V, Bonneau D, et al. Dominant optic atrophy. Orphanet J. Rare Dis. 2012; 7: 46.

Leung CKS, Weinreb RN, Li ZW, Liu S, Lindsey JD, Choi N, et al. Long-term in vivo imaging and measurement of dendritic shrinkage of retinal ganglion cells. Investig. Ophthalmol. Vis. Sci. 2011; 52: 1539-1547

Levkovitch-Verbin H. Animal models of optic nerve diseases. Eye 2004; 18: 1066-1074

Li JY, Dahlström A. Axonal transport of synaptic vesicle proteins in the rat optic nerve. J. Neurobiol. 1997; 32: 237-250.

Lingor P, Koch JC, Tönges L, Bähr M. Axonal degeneration as a therapeutic target in the CNS. Cell Tissue Res. 2012; 349: 289-311

Lingor $\mathrm{P}$, Koeberle $\mathrm{P}$, Kügler $\mathrm{S}$, Bähr M. Down-regulation of apoptosis mediators by RNAi inhibits axotomy-induced retinal ganglion cell death in vivo. Brain 2005; 128: 550-558. 
Lingor P, Teusch N, Schwarz K, Mueller R, Mack H, Bähr M, et al. Inhibition of Rho kinase (ROCK) increases neurite outgrowth on chondroitin sulphate proteoglycan in vitro and axonal regeneration in the adult optic nerve in vivo. J. Neurochem. 2007; 103: 181-189.

Lingor P, Tönges L, Pieper N, Bermel C, Barski E, Planchamp V, et al. ROCK inhibition and CNTF interact on intrinsic signalling pathways and differentially regulate survival and regeneration in retinal ganglion cells. Brain 2008; 131: 250-263.

Liu MC, Kobeissy F, Zheng W, Zhang Z, Hayes RL, Wang KKW. Dual vulnerability of tau to calpains and caspase-3 proteolysis under neurotoxic and neurodegenerative conditions. ASN. Neuro. 2011; 3: e00051.

Lunn ER, Perry VH, Brown MC, Rosen H, Gordon S. Absence of Wallerian Degeneration does not Hinder Regeneration in Peripheral Nerve. Eur. J. Neurosci. 1989; 1: 27-33.

Luo L, O'Leary DDM. Axon retraction and degeneration in development and disease. Annu. Rev. Neurosci. 2005; 28: 127-156.

Ma M, Ferguson TA, Schoch KM, Li J, Qian Y, Shofer FS, et al. Calpains mediate axonal cytoskeleton disintegration during Wallerian degeneration. Neurobiol. Dis. 2013; 56: 34-46.

Ma M, Shofer FS, Neumar RW. Model of Traumatic Axonal Injury. J. Neurotrauma. 2012; 2563: 120829074359000.

Mabuchi F, Aihara M, Mackey MR, Lindsey JD, Weinreb RN. Regional optic nerve damage in experimental mouse glaucoma. Invest. Ophthalmol. Vis. Sci. 2004; 45: 4352-4358.

Mack TG, Reiner M, Beirowski B, Mi W, Emanuelli M, Wagner D, et al. Wallerian degeneration of injured axons and synapses is delayed by a Ube4b/Nmnat chimeric gene. Nat. Neurosci. 2001; 4: 1199-1206.

De Matteis MA, Morrow JS. Spectrin tethers and mesh in the biosynthetic pathway. J. Cell Sci. 2000; 113 (Pt13): 2331-2343.

McKinnon SJ. The cell and molecular biology of glaucoma: Common neurodegenerative pathways and relevance to glaucoma. Investig. Ophthalmol. Vis. Sci. 2012; 53: 2485-2487. 
Mehdi S. Cell-penetrating inhibitors of calpain. Trends Biochem. Sci. 1991; 16: 150-153.

Merkel TC, Bondar VI, Nagai K, Freeman BD, Pinnau I. Gas sorption, diffusion, and permeation in poly (dimethylsiloxane). J. Polym. Sci. Part B Polym. Phys. 2000; 38: 415-434.

Milea D, Sander B, Wegener M, Jensen H, Kjer B, Jørgensen TM, et al. Axonal loss occurs early in dominant optic atrophy. Acta Ophthalmol. 2010; 88: 342-346.

Millet $\amalg$, Gillette MU. New perspectives on neuronal development via microfluidic environments. Trends. Neurosci. 2012; 35: 752-761.

Morales I, Sanchez A, Rodriguez-Sabate C, Rodriguez M. The degeneration of dopaminergic synapses in Parkinson's disease: A selective animal model. Behav. Brain. Res. 2015; 289: 19-28.

Morfini G, Pigino G, Opalach K, Serulle Y, Moreira JE, Sugimori M, et al. 1-Methyl-4-phenylpyridinium affects fast axonal transport by activation of caspase and protein kinase C. Proc. Natl. Acad. Sci. U. S. A. 2007; 104: 2442-2447.

Mudrakola H V., Zhang K, Cui B. Optically Resolving Individual Microtubules in Live Axons: Structure 2009; 17: 1433-1441.

Nakamura H, O'Leary DD. Inaccuracies in initial growth and arborization of chick retinotectal axons followed by course corrections and axon remodeling to develop topographic order. J. Neurosci. 1989; 9: 3776-3795.

Nesvizhskii Al, Keller A, Kolker E, Aebersold R. A statistical model for identifying proteins by tandem mass spectrometry. Anal. Chem. 2003; 75: 4646-4658.

Nikić I, Merkler D, Sorbara C, Brinkoetter M, Kreutzfeldt M, Bareyre FM, et al. A reversible form of axon damage in experimental autoimmune encephalomyelitis and multiple sclerosis. Nat. Med. 2011; 17: 495-499.

Oakley H, Cole SL, Logan S, Maus E, Shao P, Craft J, et al. Intraneuronal beta-amyloid aggregates, neurodegeneration, and neuron loss in transgenic mice with five familial Alzheimer's disease mutations: potential factors in amyloid plaque formation. J. Neurosci. 
2006; 26: 10129-10140.

Orimo S, Uchihara T, Nakamura A, Mori F, Kakita A, Wakabayashi K, et al. Axonal alpha-synuclein aggregates herald centripetal degeneration of cardiac sympathetic nerve in Parkinson's disease. Brain 2008; 131: 642-650.

Otey CA, Carpen O. Alpha-actinin revisited: a fresh look at an old question. Cell. Motil. Cytoskeleton 2004; 58(2): 104-11.

Öztürk G, Cengiz N, Erdoğan E, Him a., Oğuz EK, Yenidünya E, et al. Two distinct types of dying back axonal degeneration in vitro. Neuropathol. Appl. Neurobiol. 2013; 39: 362-376.

Park JW, Vahidi B, Taylor AM, Rhee SW, Jeon NL. Microfluidic culture platform for neuroscience research. Nat. Protoc. 2006; 1: 2128-2136.

Petzold a, Rejdak K, Plant GT. Axonal degeneration and inflammation in acute optic neuritis. J. Neurol. Neurosurg. Psychiatry. 2004; 75: 1178-1180.

Raff MC, Whitmore AV, Finn JT. Axonal Self-Destruction and Neurodegeneration. Science 2002; 296(5569): 868-71.

Ramos JW, Hughes PE, Renshaw MW, Schwartz MA, Formstecher E, Chneiweiss $H$, et al. Death effector domain protein PEA-15 potentiates Ras activation of extracellular signal receptor-activated kinase by an adhesion-independent mechanism. Mol. Biol. Cell 2000; 11: 2863-2872.

Resnikoff S, Pascolini D, Mariotti SP, Pokharel GP. Global magnitude of visual impairment caused by uncorrected refractive errors in 2004. Bull. World Health Organ. 2008; 86: 63-70.

Rhee SW, Taylor AM, Tu CH, Cribbs DH, Cotman CW, Jeon NL. Patterned cell culture inside microfluidic devices. Lab Chip 2005; 5: 102-107.

Ribas VT, Schnepf B, Challagundla M, Koch JC, Bähr M, Lingor P. Early and Sustained Activation of Autophagy in Degenerating Axons after Spinal Cord Injury [Internet]. Brain. Pathol. 2015; 25: 157-170. 
Roberts-Lewis JM, Savage MJ, Marcy VR, Pinsker LR, Siman R. Immunolocalization of calpain I-mediated spectrin degradation to vulnerable neurons in the ischemic gerbil brain. J. Neurosci. 1994; 14: 3934-3944.

Rogemond V, Auger C, Giraudon P, Becchi M, Auvergnon N, Belin MF, et al. Processing and nuclear localization of CRMP2 during brain development induce neurite outgrowth inhibition. J. Biol. Chem. 2008; 283: 14751-14761.

Rotshenker S. Wallerian degeneration: the innate-immune response to traumatic nerve injury. J. Neuroinflammation 2011; 8: 109.

Russo R, Berliocchi L, Adornetto A, Varano GP, Cavaliere F, Nucci C, et al. Calpain-mediated cleavage of Beclin-1 and autophagy deregulation following retinal ischemic injury in vivo. Cell Death Dis. 2011; 2: e144.

Saatman KE, Abai B, Grosvenor A, Vorwerk CK, Smith DH, Meaney DF. Traumatic axonal injury results in biphasic calpain activation and retrograde transport impairment in mice. J. Cereb. Blood Flow Metab. 2003; 23: 34-42.

Samantaray S, Knaryan VH, Patel KS, Mulholland PJ, Becker HC, Banik NL. Chronic intermittent ethanol induced axon and myelin degeneration is attenuated by calpain inhibition. Brain Res. 2015; 1622: 7-21.

Sasaki Y, Vohra BPS, Lund FE, Milbrandt J. Nicotinamide mononucleotide adenylyl transferase-mediated axonal protection requires enzymatic activity but not increased levels of neuronal nicotinamide adenine dinucleotide. J. Neurosci. 2009; 29: 5525-5535.

Selkoe DJ. Alzheimer's disease is a synaptic failure. Science 2002; 298: 789-791.

Shi P, Gal J, Kwinter DM, Liu X, Zhu H. Mitochondrial dysfunction in amyotrophic lateral sclerosis. Biochim. Biophys. Acta. 2010; 1802: 45-51

Shilov I V, Seymour SL, Patel AA, Loboda A, Tang WH, Keating SP, et al. The Paragon Algorithm, a next generation search engine that uses sequence temperature values and feature probabilities to identify peptides from tandem mass spectra. Mol. Cell. Proteomics 
2007; 6: 1638-1655.

Spiliotis ET. Regulation of microtubule organization and functions by septin GTPases. Cytoskeleton 2010; 67: 339-345.

Spira ME, Oren R, Dormann A, Gitler D. Critical calpain-dependent ultrastructural alterations underlie the transformation of an axonal segment into a growth cone after axotomy of cultured Aplysia neurons. J. Comp. Neurol. 2003; 457: 293-312.

Szklarczyk D, Franceschini A, Wyder S, Forslund K, Heller D, Huerta-Cepas J, et al. STRING v10: protein-protein interaction networks, integrated over the tree of life. Nucleic Acids Res. 2014; 43: D447-D452.

Tagliaferro P, Kareva T, Oo TF, Yarygina O, Kholodilov N, Burke RE. An early axonopathy in a hLRRK2 (R1441G) transgenic model of Parkinson disease. Neurobiol. Dis. 2015; 82: 359-371.

Taylor AM, Berchtold NC, Perreau VM, Tu CH, Li Jeon N, Cotman CW. Axonal mRNA in uninjured and regenerating cortical mammalian axons. J. Neurosci. 2009; 29: 4697-4707.

Taylor AM, Blurton-jones M, Rhee SW, Cribbs DH, Carl W. A microfluidic culture platform for CNS axonal injury, regeneration and transport. Nat. Methods 2005; 2(8): 599-605.

Taylor AM, Rhee SW, Tu CH, Cribbs DH, Cotman CW, Jeon NL. Microfluidic multicompartment device for neuroscience research. Langmuir 2003; 19: 1551-1556.

Tönges L, Planchamp V, Koch JC, Herdegen T, Bähr M, Lingor P. JNK isoforms differentially regulate neurite growth and regeneration in dopaminergic neurons in vitro. J. Mol. Neurosci. 2011; 45: 284-293.

Touma E, Kato S, Fukui K, Koike T. Calpain-mediated cleavage of collapsin response mediator protein(CRMP)-2 during neurite degeneration in mice. Eur. J. Neurosci. 2007; 26: 3368-3381.

Trip. Retinal nerve fiber layer axonal loss and visual dysfunction in optic neuritis. Ann. Neurol. 2005; 58: 383-391.

Tsujinaka T, Kajiwara Y, Kambayashi J, Sakon M, Higuchi N, Tanaka T, et al. Synthesis of a 
new cell penetrating calpain inhibitor (calpeptin). Biochem. Biophys. Res. Commun. 1988; 153: 1201-1208.

Vallee RB, Shpetner HS, Paschal BM. The role of dynein in retrograde axonal transport. Trends Neurosci. 1989; 12: 66-70.

Vosler PS. Calpain-Mediated Signaling Mechanisms in Neuronal Injury and Neurodegeneration. 2009; 38: 78-100.

Waller A. Experiments on the Section of the Glossopharyngeal and Hypoglossal Nerves of the Frog, and Observations of the Alterations Produced Thereby in the Structure of Their Primitive Fibres. Philos. Trans. R. Soc. 1850; 140: 423-429.

Wang D, Ayers MM, Catmull DV, Hazelwood L, Bernard CC, Orian JM. Astrocyte-associated axonal damage in pre-onset stages of experimental autoimmune encephalomyelitis. Glia 2005; 51: 235-240.

Wang JT, Medress ZA, Barres BA. Axon degeneration: Molecular mechanisms of a self-destruction pathway. J. Cell Biol. 2012; 196: 7-18.

Wang KKW. Calpain and caspase: Can you tell the difference? Trends Neurosci. 2000; 23: 20-26.

Wang X, Li Y, He Y, Liang HS, Liu EZ. A Novel Animal Model of Partial Optic Nerve Transection Established Using an Optic Nerve Quantitative Amputator. PLoS One 2012; 7(9): e44360.

William L, Povlishock JT, Graham DL, Al MET. A mechanistic analysis of nondisruptive axonal injury. J. Neurotrauma. 1997; 14(7): 419-40.

Yang J, Weimer RM, Kallop D, Olsen O, Wu Z, Renier N, et al. Regulation of axon degeneration after injury and in development by the endogenous calpain inhibitor calpastatin. Neuron 2013a; 80: 1175-1189.

Yoon SY, Choi JE, Choi JM, Kim DH. Dynein cleavage and microtubule accumulation in okadaic acid-treated neurons. Neurosci. Lett. 2008; 437: 111-115. 
You Y, Gupta VK, Li JC, Klistorner A, Graham SL. Optic neuropathies: Characteristic features and mechanisms of retinal ganglion cell loss. Rev. Neurosci. 2013; 24: 301-321.

Yousefi S, Perozzo R, Schmid I, Ziemiecki A, Schaffner T, Scapozza L, et al. Calpain-mediated cleavage of Atg5 switches autophagy to apoptosis. Nat. Cell Biol. 2006; 8: 1124-1132.

Zhang X, Jones D, Gonzalez-Lima F. Mouse model of optic neuropathy caused by mitochondrial complex I dysfunction. Neurosci. Lett. 2002; 326: 97-100.

Zhang Z, Ottens AK, Sadasivan S, Kobeissy FH, Fang T, Hayes RL, et al. Calpain-mediated collapsin response mediator protein $-1,-2$, and -4 proteolysis after neurotoxic and traumatic brain injury. J. Neurotrauma 2007; 24: 460-472.

Zhong L, Li B, Mah CS, Govindasamy L, Agbandje-McKenna M, Cooper M, et al. Next generation of adeno-associated virus 2 vectors: point mutations in tyrosines lead to high-efficiency transduction at lower doses. Proc. Natl. Acad. Sci. U. S. A. 2008; 105: 7827-7832. 


\section{Abbreviations}

AAD: acute axonal degeneration

AAV: adeno-associated virus

ACTN4: alpha-actinin 4

AD: Alzheimer's disease

AIR: axonal integrity ratio

ALS: amyotrophic lateral sclerosis

APS: ammonium persulfate

BDP: breakdown product

Blast: basic local alignment search tool

BSA: bovine serum albumin

CDC42: cell division control protein 42

CMF: calcium magnesium-free

CNS: central nervous system

$\mathrm{CO}_{2}$ : carbon dioxide

CRMP2: collapsin response mediator protein-2

DAPI: 4', 6-diamidino-2-phenylindole

DMEM: Dulbecco's Modified Eagle Medium

DMSO: dimethyl sulfoxide

DIV: days in vitro

DRG: dorsal root ganglion

DTT: dithiothreitol

Dynein IC: dynein intermediate chain

E18: embryonic day 18

EAE: experimental autoimmune encephalomyelitis

ECL: enhanced chemiluminescence

EGFP: enhanced green fluorescent protein

FAD: focal axonal degeneration 
FCS: fetal calf serum

GNB1: $\mathrm{G}$ protein beta subunit 1

GOT1: aspartate transaminase 1

HBSS: Hank's balanced salt solution

$\mathrm{H}_{2} \mathrm{O}_{2}$ : hydrogen peroxide

HRP: horseradish peroxidase

hSyn: human synapsin

IOP: intraocular pressure

LC3: microtubule-associated protein 1 light chain 3

KIF: kinesin like protein

MDH2: malate dehydrogenase 2

$\mathbf{M g C l}_{2} \cdot \mathbf{6} \mathrm{H}_{\mathbf{2}} \mathrm{O}$ : magnesium chloride hexahydrate

mPTP: mitochondrial permeability transition pore

mRNA: messenger RNA

MS: multiple sclerosis

MTS: mitochondrial targeting sequence

$\mathrm{NaCl}$ : sodium chloride

NC: nitrocellulose

NGF: nerve growth factor

NIT2: omega-amidase

NMNAT: nicotinamide mononucleotide adenylyltransferase

ONC: optic nerve crush

P1: postnatal day 1

PBS: phosphate-buffered saline

PD: Parkinson's disease

PDL: poly-D-lysine

PDMS: polydimethylsiloxane

PEA-15: astrocytic phosphoprotein-15

PFA: paraformaldehyde 
PLO: poly-L-ornithine

PVDF: polyvinylidene difluoride

QD: quantum dot

QqTOF: quadrupole/time-of-flight

RGC: retinal ganglion cell

RFP: red fluorescent protein

RNFL: retinal nerve fiber layer

RNS: reactive nitrogen species

ROS: reactive oxygen species

Rpm: rounds per minute

SDS: sodium dodecyl sulfate

SEM: standard error of the mean

SEPT2: septin 2

SPTAN1: alpha-II spectrin

SPTBN1: beta-II spectrin

STRING: search tool for the retrieval of interacting genes/proteins

TEMED: tetramethylethylenediamine

TBS-T: TBS-Tween20

Tris: 2-Amino-2-hydroxymethyl-propane-1, 3-diol

TU: transforming units

UBE4b: E4-type ubiquitin ligase UBE4b

WD: Wallerian degeneration

WIds: Wallerian degeneration slow

YWHAE: 14-3-3 protein epsilon 


\section{Acknowledgements}

I am very happy to have the chance to thank all the people who supported me during the period of my PhD study!

I would like to especially thank my close supervisor Dr. Jan C. Koch for his great supervision, patience, suggestions and encouragement!

I would like to thank my supervisor Prof. Dr. Paul Lingor for his great support and all the nice discussions!

I would like to thank Prof. Mathias Bähr for giving me the opportunity to study in his lab at the department of Neurology in University Medicine Göttingen!

I would like to thank my thesis committee members Prof. Dr. Ralf Heinrich and Prof. Dr. Michael Hörner for their suggestions and great support!

I would like to thank Prof. Dr. Uwe Michel for providing me the plasmids, viral vectors, as well as for all the kind suggestions!

I would like to thank Dr. Christof Lenz, Prof. Dr. Caroline C. Friedel, and Prof. Dr. Sarah Köster for the collaborations!

I would like to thank all my dear friends in the Lingor group: Malleswari Challagundla, Zara d’Hedouville, Eleonora Carboni, Anna E. Roser, Kim A. Saal, Lars Tatenhorst, Lars Tönges, Alexander Böcker and Vinicius T. Ribas for their help and support, as well as Lisa Barski and Vivian Dambeck for their technical help!

I would like to thank the members in the Waldweg lab, Éva Szegő for helping me to establish 
the microfluidic chamber system, as well as Barbara Müller, Sonja Heyroth and Christiane Fahlbusch for all their technical help!

I would like to thank my dear parents and sisters for their support and encouragement abroad!

Finally, I would like to thank my dear Fanzhi for his love all the time! 


\section{Curriculum vitae}

\section{Personal Information}

$\begin{array}{llll}\text { Name: } & \text { Jiannan Zhang } & \text { Nationality: } & \text { Chinese } \\ \text { Date of birth: } & \text { 19.06.1986 } & \text { Place of birth: } & \text { Tianjin, China } \\ \text { E-mail: } & \text { zhangjiannan86@gmail.com } & \end{array}$

\section{Education}

09.2004-06.2008 Bachelor study in Bioscience, School of Life Sciences, Lanzhou University, China.

Bachelor thesis "The synthesis of chiral thiourea and asymmetric Michael addition of highly functionalized ketones to nitroalkenes catalyzed by a Chiral amine-thiourea bifunctional organocatalyst" under the supervision of Prof. Rui Wang at the Institute of Biochemistry and Molecular Biology, School of Life Sciences, Lanzhou University

09.2008-06.2011 Master study in Biochemistry and Molecular biology, School of Life Sciences, Lanzhou University, China.

Master thesis "The studies on the antinociceptive effects of Neuropeptide $S$ in the mouse formalin test" under the supervision of Prof. Rui Wang at the Institute of Biochemistry and Molecular Biology, School of Life Sciences, Lanzhou University

09.2011-now PhD study in University of Göttingen, Germany

\section{Activities}

-Poster presentation during the 10th Gottingen Meeting of the German Neuroscience Society in 2013

-Poster presentation during the 9th FENS Forum of Neuroscience in 2014 


\section{Publications}

Jiang X, Zhang Y, Liu X, Zhang G, Lai L, Wu L, Zhang J, Wang R. Enantio- and diastereoselective asymmetric addition of 1,3-dicarbonyl compounds to nitroalkenes in a doubly stereocontrolled manner catalyzed by bifunctional rosin-derived amine thiourea catalysts. $J$ Org Chem. 2009; 74(15):5562-7.

Li W, Chang M, Peng YL, Gao YH, Zhang JN, Han RW, and Wang R. Neuropeptide S produces antinociceptive effects at the supraspinal level in mice. Regul Pept. 2009;156:90-5.

Peng $\mathrm{YL}^{1}$, Zhang $\mathrm{JN}^{1}$, Chang M, Li W, Han RW, Wang R. Effects of central neuropeptide $\mathrm{S}$ in the mouse formalin test. Peptides. 2010; 31(10):1878-83. (Co-first author)

Koch JC, Bitow F, Haack J, d'Hedouville Z, Zhang JN, Tönges L, Michel U, Oliveira LM, Jovin TM, Liman J, Tatenhorst L, Bähr M, Lingor P. Alpha-Synuclein affects neurite morphology, autophagy, vesicle transport and axonal degeneration in CNS neurons. Cell Death Dis. 2015. doi: 10.1038/cddis.2015.169.

Zhang JN, Michel U, Lenz C, Friedel CC, Köster S, d'Hedouville Z, Tönges L, Urlaub H, Bähr M, Lingor P, and Koch JC. Calpain-mediated cleavage of collapsin response mediator protein-2 drives acute axonal degeneration. Submitted (Under review). 